\title{
Time-consistent and market-consistent actuarial valuations
}

Citation for published version (APA):

Salahnejhad Ghalehjooghi, A. (2016). Time-consistent and market-consistent actuarial valuations.

[Doctoral Thesis, Maastricht University]. Maastricht University. https://doi.org/10.26481/dis.20161221as

Document status and date:

Published: 01/01/2016

DOI:

10.26481/dis.20161221as

Document Version:

Publisher's PDF, also known as Version of record

Document license:

Unspecified

\section{Please check the document version of this publication:}

- A submitted manuscript is the version of the article upon submission and before peer-review. There can be important differences between the submitted version and the official published version of record.

People interested in the research are advised to contact the author for the final version of the publication, or visit the DOI to the publisher's website.

- The final author version and the galley proof are versions of the publication after peer review.

- The final published version features the final layout of the paper including the volume, issue and page numbers.

Link to publication

\footnotetext{
General rights rights.

- You may freely distribute the URL identifying the publication in the public portal. please follow below link for the End User Agreement:

www.umlib.nl/taverne-license

Take down policy

If you believe that this document breaches copyright please contact us at:

repository@maastrichtuniversity.nl

providing details and we will investigate your claim.
}

Copyright and moral rights for the publications made accessible in the public portal are retained by the authors and/or other copyright owners and it is a condition of accessing publications that users recognise and abide by the legal requirements associated with these

- Users may download and print one copy of any publication from the public portal for the purpose of private study or research.

- You may not further distribute the material or use it for any profit-making activity or commercial gain

If the publication is distributed under the terms of Article $25 \mathrm{fa}$ of the Dutch Copyright Act, indicated by the "Taverne" license above, 


\title{
TIME-CONSISTENT AND MARKET-CONSISTENT ACTUARIAL VALUATIONS
}

\author{
DISSERTATION \\ to obtain the degree of Doctor at \\ Maastricht University, \\ on the authority of the Rector Magnificus, \\ Prof. dr. Rianne M. Letschert, \\ in accordance with the decision \\ of the Board of Deans, \\ to be defended in public \\ on Wednesday December 21, 2016 at 10:00 hours
}

by

Ahmad Salahnejhad Ghalehjooghi 


\section{Supervisor:}

Prof. dr. Antoon Pelsser

\section{Co-supervisor:}

Dr. Thomas Post

\section{Assessment Committee:}

Prof. dr. Dennis Bams (chairman)

Prof. dr. Jan Dhaene (Katholieke Universiteit Leuven, Belgium)

Prof. dr. Peter Schotman

Prof. dr. Michel Vellekoop (Universiteit van Amsterdam)

\section{绝HPCFìnance}

The research leading to these results has received funding from the European Union Seventh Framework Programme ([FP7/2007-2013] [FP7/2007-2011]) under grant agreement $n^{\circ}$ 289032.

ISBN 978-94-6233-494-6

(C) Ahmad Salahnejhad Ghalehjooghi, 2016.

All rights reserved. No part of this publication may be reproduced, stored in a retrieval system, or transmitted, in any form or by any means, electronically, mechanically, photocopying, recording or otherwise, without prior permission of the author. 


\section{Contents}

Contents $\diamond \mathrm{i}$

1 Introduction $\diamond 1$

1.1 Market-consistent Valuation $\diamond 2$

1.2 Time-consistent Valuation $\diamond 4$

1.3 Pension Valuation $\diamond 8$

1.4 How to Read this Thesis $\diamond 11$

2 Time-Consistent Actuarial Valuations under Diffusion Processes $\diamond$ 13

2.1 Time-Consistent Valuation Operators $\diamond 14$

2.2 Variance Pricing $\diamond 16$

2.3 Standard-Deviation Pricing $\diamond 23$

3 Time-Consistent Actuarial Valuations under Jump-Diffusion Processes $\diamond 29$

3.1 Time-consistent Pricing under Jump Process $\diamond 30$

3.2 Numerical Example $\diamond 38$

4 Market-Consistent Valuation under EIOPA Risk-margin and Timeconsistency $\diamond 49$

4.1 Market Consistent Valuation $\diamond 50$

4.2 Two-step Pricing through Binomial Discretization $\diamond 61$

5 Market-Consistent Pricing for Unit-linked Contract $\diamond 69$

5.1 Market-Consistent Valuation for Unit-linked Contract $\diamond 70$

6 Market-Consistent Valuation of the Participating Pension Contract $\diamond 87$

6.1 Model and Setup $\diamond 88$

6.2 Pricing Framework $\diamond 95$

6.3 Numerical Method $\diamond 99$

6.4 Numerical Results $\diamond 105$ 
7 Conclusions $\diamond 117$

7.1 Time-consistent Valuations $\diamond 117$

7.2 Market-consistent Valuations $\diamond 118$

7.3 Market-consistent Valuation of the Participating Pension Policy $\diamond 119$

7.4 Perspectives for Future Research $\diamond 120$

Summary $\diamond 131$

Acknowledgements $\diamond 133$

About the Author $\diamond 135$ 


\section{1 \\ Introduction}

During the last decades the insurance industry started an evolutionary movement from a separated identity in the business to integrate more and more into the financial markets. Traditionally, the actuaries were conducting the risk management and technical calculations in the insurance companies in a very special and (to some extent) isolated methodologies. Even being an actuary was extremely unknown carrier with very special skills and particular ethical values consistent with the professional requirements of this carrier. Actuaries had their own methods to measure the associated risks which was sometimes very advanced and structurally different than the financiers' methodologies in financial markets. Among all, some of the main reasons for such difference were: the nature of the risks, market mechanism, regulations, and the way the insurance companies were taking the responsibilities to handle the liabilities, comparing to pure financial institutions like banks. For example, actuaries were dealing with the time-value of money via the concept of the "technical interest rate", while financiers were doing the same via the term-structure of the interest rate from the market and stochastic models.

However, recent evolution of the trading and business in the world, required the insurance institutions to stay connected to the other financial sectors and accordingly adapt their activities to the market dynamics. during 80's and 90's there was a great effort to mix the stochstic models in finance into the established actuarial methods (See Darcy (1989)). This was the time that some researchers started to study new methods to measure and identify the insurance and financial risks in a unified framework. The main ideas started by Yaari (1987) and was developed on pricing aspect by a series of the papers written by different researchers. Most of the studies were focused on pricing insurance liabilities by financial pricing operators (See Cummins (1990). This was motivated by some similarities between the insurance contracts such as "stop-loss" re-insurance and the financial derivatives like the European call option for the underlying stocks in the financial markets. In the unified method, there was a great deal of attention to the no-arbitrage argument for the insurance risks; See for example Schmeidler (1989) and Venter (1991). The studies continued by using "distortion operators" to unify the pricing operators for both financial and 
insurance risks. See for example, Wang (2002) and Hamada and Sherris (2003).

Furthermore, the insurers had to synchronize their reporting and accounting information with the rest of the financial institutions. The regulators started to define new regulatory measures for the insurers to enforce the so called integration which would result in more clear and understandable picture of their liabilities and financial status. The idea became identified with the concept of the "market-consistent" valuation where the regulator demands to capture and use all the related market information and dynamics to valuate the non/less-tradable insurance liabilities in the incomplete markets.

\subsection{Market-consistent Valuation}

Life insurance companies have liabilities with very long-dated maturities on their books. Most people start saving for their pensions from age 25. People are expected to live to age 85 , with the oldest individuals living to age 115. Hence, pension funds and life insurance companies are facing contractual obligations that can easily last on average for 60 years and, sometimes, even 80-90 years in the future. Therefore, the valuation and risk management of these very long-dated contracts is an important problem. To provide a sense of the magnitude of the problem: for life insurance and pension products, an approximate $20 \%$ of the NPV of the cash flows is in the "tail" that is 30+ years long. Most of these very long-dated contracts are not (actively) tradable in the market (non-market risks); therefore, no other related contracts can be used to hedge these risks. Moreover, these contracts also have significant exposure to market risks, such as interest rates, and inflation risks. Therefore, pricing and risk management of such liabilities require a valuation method that considers both financial and non-financial risks. The insurance and pensions' regulator EIOPA and the Solvency II framework required methods, such as "market-consistent" valuation, which should also include explicit loading for non-market risks.

Typically, actuarial pricing (through premium principles) operators ignores the mechanisms of the financial markets and hedging strategies, whereas classical financial pricing methods also ignore the unhedgeable risks available in a hybrid payoff or portfolio. In contrast, actuarial pricing methods usually valuate risk in a "static" manner through a one-period pricing procedure that ignores the mid-time evolution of the insurance risk drivers. Yet, in a "dynamic" manner, financial pricing operators account for the evolution of risk during the time to maturity. This consideration is necessary to capture the "pathdependent" nature of the payoffs in a broad range of the traded financial contracts. Using market-consistent actuarial valuation, we integrate the financial and actuarial pricing methods. The setup allows us to consider both hedgeable and unhedgeable risks, including their interactions, and price a combined payoff in a dynamic manner.

From a theoretical perspective, pricing a long-dated life/pension liability is a pricing 
problem in an "incomplete market" because a pension contract is exposed to hedgeable financial risks and non-hedgeable actuarial risks. When faced with an incomplete market, the standard risk-neutral Black-Scholes pricing machinery breaks down because it is no longer possible to construct a perfect replicating portfolio that hedges all risks. Therefore, we need to consider pricing methods that explicitly consider non-hedgeable risks but also that remain market-consistent in the sense that the prices of "pure" financial contracts are still consistent with risk-neutral pricing. This investigation connects us to the literature that studies risk measures/valuations in a so-called market-consistent setting. Such studies started by pricing contracts in an incomplete market setting. In this setting, one seeks to extend arbitrage-free pricing operators (only defined in a complete market setting) to the larger space of (partially) unhedgeable contracts. The paper by Hodges and Neuberger (1989) is frequently cited as the root idea of the utility-indifference pricing literature previously mentioned. A related branch of the literature extends arbitrage-free pricing operators using (local) risk-minimization techniques and the related notion of minimal martingale measures; see Föllmer and Schweizer (1989), Schweizer (1995), and Delbaen and Schachermayer (1996). A rich duality theory has been developed that makes deep connections between utility maximization and risk minimization over the martingale measures; see Cvitanic and Karatzas (1992) and Kramkov and Schachermayer (1999). For a very elegant summary, we refer to Rogers (2001).

Using utility-indifference (and duality) methods, the market consistency of the pricing operators is automatically induced. However, an explicit formal definition of the marketconsistent pricing operators has begun to emerge; see Kupper et al. (2008) and Malamud et al. (2008).

To implement a market-consistent valuation, Pelsser and Stadje (2014) introduced a method called "Two-step Market Evaluation" with an axiomatic characterization for that including a "market local property." The importance of the study was that, in light of some theoretical results and under certain assumptions (normally available for the standard actuarial premium principles), it proved that the two-step market evaluation can turn any conditional valuation operator into a market-consistent version. In general, the two-step market evaluation does the following:

- First step: Conditions the general payoff/position on a financial risk driver and applies an actuarial pricing operator that turns the payoff into a function of only financial risk that through the structure, is perfectly hedgeable;

- Second step: Applies the conditional expectations under a unique equivalent martingale measure $\mathbb{Q}$ that reflects the no-arbitrage argument for the hedgeable part of the general position.

Before this, Musiela and Zariphopoulou (004b) also used a similar method to calculate the indifference premium through an exponential utility function in an incom- 
plete market by first conditioning the premium on financial trading. Møller (2002) also worked on hedging and the valuation of insurance risks combined with financial risks, and used different methods, such as risk minimization, mean-variance hedging, and superreplication, to valuate them.

\subsection{Time-consistent Valuation}

An important requirement of a market-consistent pricing operator is "time-consistency". Time-consistency implies that the value order of the different positions measured using a dynamic price operator in the future time $t$, is consistent with their order at any prior time $s<t$. When we compute the price of a contract with a payoff in the future, we cannot simply price a contract at $t=0$ and then "forget" about it for the rest of the period. Instead, we should follow the contract over time and must update our pricing and hedging position when the new information arrives in the market. The updated prices must be time-consistent to avoid arbitrage opportunities. Moreover, insurance companies need to "re-valuate" their liabilities and assets in certain periods for different reasons, such as asset liability management and supervision purposes. The importance of the problem is more when the insurance companies have very long-dated liabilities, such as annuities and pension products, in their portfolios. Hence, the pricing method should be adjusted to consider such a revaluation process.

Pricing under time-consistent valuation requires a dynamic setting for the price operators. Pelsser and Stadje (2014) also showed that, under certain assumptions, market consistency and a market local property also hold in a dynamic setting using a timeconsistency property. In addition, they proved that for finitely many stopping times $\tau \in[0, T]$ of the underlying insurance process, time consistency and market consistency imply that every evaluation (including actuarial premium principles) must admit a representation of the two-step market evaluation. Note that the result is stable even when the insurance process follows a jump diffusion process, and jumps occur only at finitely many predictable times.

Standard actuarial premium principles usually consider a static premium calculation problem: what is today's price of an insurance contract with payoff at time $T$. Textbooks such as those by Bühlmann (1970), Gerber (1979), and Kaas et al. (2008) provide examples of this. The study of risk measures and the closely related concept of the monetary risk measures have also been studied in static settings by authors such as Artzner et al. (1999) and Cheridito et al. (2005). The study of utility-indifference valuations has mainly confined itself to static settings as well. Different applications can be found in papers by Young and Zariphopoulou (2002), Henderson (2002), Hobson (2004), Musiela and Zariphopoulou (2004) and Monoyios (2006), and the book by Carmona (2009). 
Financial pricing usually considers a "dynamic" pricing problem, and looks at how the price evolves over time until the final payoff date $T$. This dynamic perspective is driven by the focus on hedging and replication. The literature was started by the seminal paper of Black and Scholes (1973) and has been immensely generalized to broad classes of securities and stochastic processes; see Delbaen and Schachermayer (1994). Some researches in the last two decades focus on combining actuarial and financial pricing. See for example, Wang (2002) where he used distortion risk measures to price both types of risks and Goovaerts and Laeven (2008) where they used actuarial risk measures to price financial derivatives.

In the last decade, researchers have begun to investigate risk measures in a dynamic setting, to construct a time-consistent risk measures See Riedel (2004), Cheridito et al. (2006b),Cheridito et al. (2006a), Rosazza Gianin (2006) and Artzner et al. (2007) for the general ideas on the dynamic risk measures. Concerning the time-consistency in continuous time, see Peng (2004), Frittelli and Gianin (2004), Maccheroni et al. (2006), BionNadal (2009), and Barrieu and El Karoui (2009). As an example, Stadje (2010) showed how a large class of dynamic convex risk measures in continuous-time can be derived from the limit of their discrete time versions. Moreover, Jobert and Rogers (2008) showed how time-consistent valuations can be constructed through the backward induction of static one-period risk measures (or "valuations"). In fact, Jobert and Rogers (2008) showed that a time-consistent price can be obtained by sticking the shorter one-period static price operators to each other to determine a price over a longer period. Although the real revaluation of liabilities occurs in a forward manner, the compatible pricing method can reflect this property in a backward valuation manner, starting from maturity. Therefore, we need to price the payoffs and contracts by re-valuating the value of the payoff in middle times in a backward manner and reaching a price at $t=0$. Later, Pelsser and Stadje (2014) studied time and market consistency of the well-known actuarial principles in a dynamic setting by using a two-step valuation method.

Insurance risk can be modeled in a stochastic way by using a diffusion process. However, it is usual that insurance risks exhibit also jump type movements in their evolution, and the data usually contain a number of extreme events, and stylized facts usually exist such as fat-tailed and skewed distributions. This justifies the usage of a jump component to draw a realistic inference about the dynamic pricing framework. Merton (1976) introduced the jump-diffusion model to price options by assuming discontinuity in returns. The model was developed extensively for financial modeling, actuarial valuation and the pricing of different derivatives and contingent claims in incomplete markets. There are numerous works about the jump process in finance; see for example Cont and Tankov (2012). For an introduction to the application of diffusion and jump processes in insurance see, for example, Korn et al. (2010) and for more specific actuarial applications see Biffis (2005), Verrall and Wüthrich (2012), Chen and Cox (2009), and Jang (2007). Some re- 
searchers have generalized the concept of time-consistent dynamic risk measures by using jump-diffusion processes when underlying risks include jumps. See for example BionNadal (2008). The idea was developed in actuarial valuation using Backward Stochastic Differential Equations (BSDE) and $g$-expectations as more powerful tools to deal with non-linear pricing operators such as different premium principles. There are also a number of studies about modeling jumps with BSDEs in valuation and portfolio choice. See for example the textbook by Delong (2013) and the paper by Laeven and Stadje (2014).

In this thesis, we investigate the well-known actuarial premium principles such as the Variance principle and the Standard-Deviation principle, and we study their timeconsistent extension. We first consider one-period valuations, then extend this to a multiperiod setting using the backward iteration method of Jobert and Rogers (2008) for a given discrete time-step $(t, t+\Delta t)$, and finally consider the continuous-time limit for $\Delta t \rightarrow 0$. A more general setting to model the insurance risk could be "infinite activity Lévy process" where it allows for infinite number of jumps for any finite time interval. However, as it does not seem realistic for an insurance process to have infinite number of jumps when $(t, t+\Delta t)$ is infinitesimally small, we waive the infinite activity Lévy process and we focus on investigating the method with simple diffusion and jump-diffusion processes.

We apply the backward iteration to a simple diffusion model to show that the oneperiod Variance premium principle converges to the non-linear exponential indifference valuation. Furthermore, we study the continuous-time limit of the one-period StandardDeviation principle and the Cost-of-Capital principle, and establish that in the diffusion setting, they converge to the same limit represented by an expectation under an equivalent martingale measure. We apply the same approach to the jump-diffusion setting and show that the time-consistent prices for different premium principles in the limit converge to different results than in the diffusion case. We mainly used the infinitesimal generator together with Itô's formula for different forms of the premium with the underlying process $y(t)$ in both diffusion and jump-diffusion models. See for example the book by Shreve (2010) about martingales and Itô's formula and the book by Øksendal (2003) for infinitesimal generators. As an exception, in the Cost-of-Capital principle under the jump setting, we have to make inference about the distribution of the insurance process under VaR operator. To do so, we will assume the jump process as a special case of the Lévy process and find its characteristic function. To get more insight about the Lévy process and its applications, see for example Figueroa-López (2012) and the textbook by Barndorff-Nielsen et al. (2001). We apply this method to a health process to price a stylized life insurance product and we use a Markov chain approximation to discretize the time and state space of the underlying insurance process. See for example Kushner and Dupuis (2001), Duan et al. (2003), and Tang and $\mathrm{Li}$ (2007) for the idea of using a Markov chain approximation to price contingent payoffs in theory and application. 


\section{Market-consistent Valuation in Other Alternative Frameworks}

Apart from the time-consistency argument, the market-consistent valuation can also be implemented for some other alternative valuation frameworks. Therefore, as one other contribution of this thesis, we provide an implementation of the market-consistent actuarial price using a two-step valuation method in "one-period" valuation setting, EIOPA's risk margin method, besides the time-consistent setting. Furthermore, we use binomial discretization to present a continuous-time limit of the TCMC prices for the Variance and Standard-Deviation actuarial premium principles. We use the result of the contributions in the time-consistent actuarial valuation and the similar approach to obtain these results.

We apply the implementation to price a stylized life insurance contract. We work on a simple unit-linked contract without guarantee that it has enough flexibility to present a realistic picture of the method in a simple manner, and apply the two-step actuarial valuation on both one-period and multi-period settings. We also provide a numerical scheme to implement the two-step actuarial valuations regardless of the dependence structure of the financial and actuarial risks. The contract has two main risk drivers: the market value of the assets/equity as the financial risk and the longevity risk of an individual or a cohort entered in the contract as the actuarial risk. The market value of the asset or equity is modeled using Geometric Brownian Motion (GBM) for pricing in a complete market with a noarbitrage argument. Longevity risk is modeled using the famous Lee-Carter model introduced by Lee and Carter (1992). The Lee-Carter model studies the mortality trends with a time-specified stochastic index and combine it with age-specified average trend and sensitivity coefficient to formulate the projected force of mortality. The model is widely used in applications as a discrete-time (normally annual) model. Among other extensions, Renshaw and Haberman (2006) extend the Lee-Carter model by adding a cohort-based factor to it. On the other hand, Cairns et al. (2006) model stochastic mortality in a continuoustime setting similar to the model for the positive interest rate risk. They use the model to price mortality-indexed products under the no-arbitrage condition. Cairns et al. (2009) and Cairns et al. (2011) later compare different stochastic mortality models and their results on mortality data of different countries. As we consider a discrete-time framework, and because it is widely used in the industry, we use the Lee-Carter model to investigate the mortality/longevity risk. Both models are constructed in a simple diffusion setting without jump. In the end, we compare the TCMC price with the market-consistent version of the EIOPA risk-margin price and the discounted expected value, also called the "Best-estimate price."

Most studies assume independence between financial and actuarial risks. We use this standard assumption to discuss and provide analytical solutions. However, some studies discussed the evidence on the relationship between the economic and the financial factors on one hand, and the mortality/longevity factor in the other hand. This is consistent with 
the notion of a market-consistent valuation that uses this dependence to partially hedge a position. See, for example, Hanewald et al. (2011), who developed a dynamic asset-liability model on the basis of the link between macroeconomic (and, accordingly, the interest rate and stock price) fluctuations and the Lee-Carter model.

\subsection{Pension Valuation}

We are interested to apply the findings of this research and the market-consistent valuation to pension liabilities. Pension contract valuation is significantly related to different risk factors that are involved in assets and liabilities of a pension fund or insurance company. Simply put, the money collected by the pension fund from the policyholders, is typically invested in different financial markets such as equities, bonds, and properties like real states and they all together form the asset side of the balance sheet. On the liability side, the main factor is the reserves to compensate for the future benefits to policyholders, apart from the bonus reserve or the buffer of the fund. The value of the pension policy and its payoff predominantly depends on the return on the asset side and the ratio of the assets to liabilities.

In relation to the assets invested in equities, some of the main risk factors that would affect the pension fund are volatility, interest rate risk, and market risks. The fund is also exposed to credit risk via investments in bonds that may default, and to liquidity risk on assets such as real estate and other properties. On the liability side, the longevity risk related to the future life of the policyholders plays the main role. Moreover, economic factors such as inflation and wage growth directly affect liabilities and can indirectly affect investment assets.

The payoffs of most modern forms of life insurance and pension contracts, such as participating policies, annuities, unit-linked plans, and pension schemes, are a mixture of the above-mentioned risk factors. These factors are stochastic and can be classified into two main categories: financial and actuarial risks. In this study, the financial risks that we consider are the equity risk with constant volatility and stochastic interest rate, and the only actuarial risk we consider is mortality/longevity.

Pension liabilities are not commonly traded in the market and are therefore (partially) unhedgeable. Nevertheless, in recent years, regulators have started to recommend the assessment of the market value of the liabilities to insurance companies and pension funds, even though there is no liquid market for them ${ }^{1}$. Since there is an underlying unhedgeable actuarial risk (mortality/longevity) in pension contracts, we consider the actuarial value of the contract instead of the arbitrage-free assumption in the pricing method. However, on the other hand, the financial risk drivers in the contract payoff must be addressed

\footnotetext{
${ }^{1}$ In some countries the market-consistent valuation has become a requirement. For example in the Netherlands, the Dutch central Bank (DNB) has imposed such a requirement.
} 
through a financial pricing framework. After all, if there is any correlation between actuarial and financial risks, the price must reflect the possible partial hedging for the actuarial risk through financial market dynamics. This is ensured by the market-consistency condition of the pricing operator. Some researchers consider the above conditions in the explicit definition of the market-consistency for pricing operators that we mentioned in previous section.

On the other hand, pension contracts impose very long-dated liabilities on the issuer. During such a long period, pension liabilities are affected by substantial social, economic, and financial shocks. If we focus on the effect of financial and actuarial risks over such a long-term valuation period, the price of the pension contract should capture and reflect market dynamics in the medium-term on the liability value. For example, when the contract includes the bonus or surrounding option, under market-consistency, the contract should be re-valuated to incorporate the market-risk factors in the medium term. To reflect such re-valuation over the valuation period, the price must be "time-consistent". Time-consistency implies that if the pension liability position $(A)$ is more expensive than position $(B)$ at a (long-dated) maturity, it is then also more expensive at any time prior to that point. Hence, to obtain time-consistency, "dynamic" valuation should be used to price the pension contract.

We focus on the valuation of the participating pension policy (PPP). A participating policy is normally characterized by a guaranteed annual return plus bonus option, which can be distributed under a special mechanism related to the ratio of the assets and liabilities. Moreover, most of the participating policies offer a surrender option to sell the policy back to the pension fund or insurance company and receive the surrender value. Several researchers have attempted to model the value of the pension contract with different underlying risk factors. Grosen and Jorgensen (2000) valuated a contract with surrounding option by using a stochastic return on investment asset in a Black-Scholes economy with constant interest rate and guaranteed return in valuation. Zaglauer and Bauer (2008) developed the model by adding stochastic interest rate and uses the Monte Carlo method to value the contract. They realized that the embedded options in the contract are considerably sensitive to make the constant interest rate stochastic. Bernard et al. (2005) extended a similar model by incorporating the default risk of the issuer and use the least Square Monte Carlo (LSMC) method for valuation. Tanskanen and Lukkarinen (2003) provided the fair value of the contract with more flexibility on the bonus option in the model. For more on valuation of the participating policies, see Kleinow (2009) and Bacinello (2003). The majority of the above-mentioned literature considers pricing contracts in an arbitrage-free condition, which implicitly assumes a liquid market for liabilities, even though such a market does not exist.

We calculate the market-consistent actuarial price of the participating policy under time-consistency arguments. We apply the two-step market evaluation of Pelsser and 
Stadje (2014) that we introduced earlier to achieve the market-consistency for actuarial premium principles, . This framework can be applied to any hybrid payoff with underlying financial and actuarial risks.

We assume that the hybrid payoff is a combination of the investment on equity, interest rate, and the mortality/longevity risks; for simplicity, we assume that volatility is constant. For the participating contract, the development comparing to the simple unitlinked contract menstione dearlier is that, instead of two underlying risk driver, now we have three risks that includes the stochastic interest rate as the second financial risk. Under the two-step actuarial valuation, we model the investment asset in a Black and Scholes (1973) framework with a Geometric Brownian motion (GBM) where there exists a unique martingale measure $\mathbb{Q}$ equivalent to the real-world measure $\mathbb{P}$, under which the price is a conditional expectation of the discounted payoff. Due to flexibility and adaptability to discretization schemes, among a wide variety of models, we choose the Hull and White (1996) short rate model for the interest rate risk. We will stay with the Lee-Carter model for the mortality/longevity risk. We show to practitioners that the method outlined to calculate the market-consistent value, is easy to implement for practical problems. All risk drivers are assumed to follow a diffusion process.

To numerically calculate the price of the contract, we focus on the finite difference intervals as the main setup for discretization of the underlying process and specify the payoff functions accordingly. To choose the most feasible numerical approach, we consider the fact that the bonus and interest crediting mechanism in participating policy results in a path-dependent process for the policy reserve. ${ }^{2}$ On the other hand, backward iteration of the conditional one-period price operator over the long-term valuation period imposes a huge load on the calculation. In particular, the higher dimension of the underlying risk drivers makes the situation worse and leads to exploding calculations of this dynamic valuation. In order to generate more speed and efficiency, Carriere (1996) and Longstaff and Schwartz (2001) used the Least Square Monte Carlo (LSMC) method to price American options. The method is widely used and developed as an efficient numerical method in theory and application. For a well-known reference on LSMC see the book by Glasserman (2004) and papers by Glasserman and Yu (2004b) and Glasserman and Yu (2004a). As regards life and pension liabilities, see Angelis et al. (2014) for use of the lattice method to discretize the investment asset and interest rate in participating policies, and Bacinello et al. (2011) for valuation of the variable annuities with LSMC. For the application of LSMC to value the participating contract and its surrounding options, see Bacinello et al. (2010), Li and Szimayer (2014), and Létourneau and Stentoft (2014).

\footnotetext{
${ }^{2}$ If the participating policy includes a surrender option as another stochastic event, where the policyholder may find it more beneficial to sell back the contract than keep it, the policy reserve is strongly path-dependent. In this sense, the contract is similar to an American put option.
} 


\subsection{How to Read this Thesis}

The common theme in this thesis is providing a pricing methodology that delivers a time-consistent and market-consistent value of the insurance liabilities, where timeconsistency is considered to secure the no-arbitrage argument over the valuation period. The liabilities are considered as a hybrid function of the financial and actuarial risks. Under certain conditions such as completeness of the financial market, we show that it is computationally feasible to price such hybrid contracts in an incomplete market setting for the actuarial risk, with the time-consistent and market-consistent (TCMC) pricing operators.

In the first part of this thesis, we focus on the time-consistent valuation where we only consider the actuarial risk in an incomplete market. In Chapter 2, Section 2.1, we define the time-consistent valuation operators and explain about the backward iteration method used to construct it. In Section 2.2, we derive the time-consistent extension of the Variance premium principle with and without discounting. Section 2.2 also includes a benchmark version of this premium and the Mean-Value principle as a more general pricing rule. In Section 2.3, we derive the time-consistent value of the Standard-Deviation and Cost-of-Capital premium principles. In both sections, we assume that the underlying pure insurance risks follow a diffusion process and we represent the results by means of the related Partial Differential Equation (PDE). In Chapter 3, Section 3.1, we assume that the underlying process includes a Poisson jump component and we derive the timeconsistent value for the principles (that we used in sections 2.2 and 2.3) in the form of the Partial Integro-Differential Equations (PIDEs). In Section 3.2, we provide an example of the pricing procedure for a stylized insurance product using the Markov chain method and show the convergence of the numerical algorithm to analytical solutions.

After we provide the result of our study on the time-consistent valuation, we introduce the market-consistent actuarial valuation setting by the two-step market valuation operator. In Chapter 4, Section 4.1, we discuss the general setup and assumptions for the two-step valuation using two different filtrations for a mixed payoff. We then extend the two-step valuation in a one-period setting to the EIOPA risk-margin price and the timeconsistent price. In Section 4.2, using a binomial discretization of the underlying financial and actuarial risks and under time consistency, we calculate a continuous-time limit of the two-step actuarial valuation and deliver the solutions through partial differential equations. In Chapter 5, section 5.1, we construct a two-step operator for a unit-linked payoff and provide some insights into the formulation of EIOPA and time-consistent prices under the independence assumption for equity and longevity risks. In Section 5.1, we introduce the Least Square Monte Carlo (LSMC) method as the related numerical method to calculate the two-step actuarial price with respect to time consistency and EIOPA risk margin. We also provide the results for these prices together with the best-estimate (expected dis- 
counted) value and the effect of dependence on price.

The last part of this thesis is devoted to time-consistent and market-consistent actuarial valuation of the participating pension contract. In Chapter 6, in Section 6.1 we introduce the participating contract and models for its underlying risk drivers. Then, we explain the liability dynamics and define the new hybrid crediting mechanism that is built based on the Grosen-Jorgensen framework, which forms the hybrid policy reserve in the final payoff. We conclude the section by providing the dynamics of the survival probability and the technical profit-sharing component in the hybrid crediting mechanism under the Lee-Carter model. In Section 6.2, we explain the pricing framework by introducing the two-step actuarial operator and executing the backward-iteration of the one-period valuation on the two-step operator. This is done to achieve a time-consistent price. Section 6.3 discusses the suggested numerical method to implement the pricing framework. The first part of this section is about simulating the hybrid policy reserve and the crediting interest rate. Then, we explain the regression-based method and adapt a version of this method that corresponds to the needed quantities in the hybrid profit-sharing mechanism of the participating contract. Finally, we formulate the calculation of the time-consistent twostep Standard-Deviation price by using the regression-based method. In Section 6.4, we provide the results of the numerical procedure to price the participating contract. In the first subsection, we deliver the time-consistent and market-consistent price and compare it with the expected value price and the one-period market-consistent price. We then examine the sensitivity of the price to the volatility of the investment asset, the parameters of the funding policy, and the randomness of the interest rate risk.

We conclude in Chapter 7 by summarizing and giving a concise technical review on the contributions of this research based on the chapters' order. We will also provide some directions, hopefully for future research. 


\section{2 \\ Time-Consistent Actuarial Valuations under Diffusion Processes}

\section{This chapter is based on:}

Pelsser, A. and Salahnejhad Ghalehjooghi, A. (2016), Time-consistent Actuarial Valuation, Insurance Mathematics and Economics, Vol 66, pp:97-112.

Time-consistent valuations (i.e. pricing operators) can be created by backward iteration of one-period valuations. In this paper we investigate the continuous-time limits of wellknown actuarial premium principles when such backward iteration procedures are applied. This method is applied to an insurance risk process in the form of a diffusion process. We show that in the case of the diffusion process, the one-period time-consistent Variance premium principle converges to the non-linear exponential indifference price. Furthermore, we show that the Standard-Deviation and the Cost-of-Capital principle converge to the same price limit. 


\subsection{Time-Consistent Valuation Operators}

Let $(\Omega, \mathscr{F}, \mathbb{P})$ be the underlying probability space and $X(\omega)$ and $Y(\omega)$ be the stochastic insurance risk processes defined over the $\sigma$-algebra $\mathscr{F}$. Indexing for the time $0 \leq t \leq T$, we form the filtration $\mathscr{F}_{t}$ as the collection of the $\sigma$-algebras. In this paper, we limit ourselves to the square integrable functions and denote the space of such random variables as $\mathscr{L}^{2}\left(\Omega, \mathscr{F}_{t}, \mathrm{P}\right)$.

Time consistency postulates that the order of riskiness of different portfolios measured by a dynamic risk measure in the future time is consistent with their riskiness at any time prior to that point in time and remains the same. It suggests that if any time $t$ the position $A$ forms a higher risk than position $B$, the level of risk will be higher for all $s<t$. The next definition formulates the time consistency of a risk measure.

Definition 2.1.1. A dynamic risk measure $\left(\rho_{t}\right)$ is Time-Consistent if and only if, for all $0 \leq$ $t \leq T$ and $\forall X, Y \in L^{2}\left(\mathscr{F}_{t}\right)$,

$$
\rho_{T}(X) \leq \rho_{T}(Y) \quad P-\text { a.s. } \Rightarrow \rho_{t}(X) \leq \rho_{t}(Y) \quad P-\text { a.s. }
$$

or equivalently by its "recursive" form for $\forall s=\Delta t, 2 \Delta t, \ldots, T-t$, we have $\rho_{t}=\rho_{t}\left(-\rho_{t+s}\right)$,

where $\rho_{t}: \mathscr{L}^{2}\left(\mathscr{F}_{T}\right) \rightarrow \mathscr{L}^{2}\left(\mathscr{F}_{t}\right)$ is a conditional risk measure for all $T \geq t$. The definition for non-negative risks (e.g. insurance losses) then becomes,

$$
\rho_{t}=\rho_{t}\left(\rho_{t+s}\right)
$$

Similar notions of time consistency can be found in Föllmer and Penner (2006), Cheridito and Stadje (2009), and Acciaio and Penner (2011).

We construct the time-consistent valuation operators for the insurance risks by the recursive form (4.1.9) and we use the backward induction method introduced by Jobert and Rogers (2008). In general we assume that the insurance process evolves during the time period $[0, T]$ and that at maturity time $T$ it falls into a bounded state space where we can also define the state space of the contingent payoff. Based on this method, time consistency can be achieved for the price operator by decomposing the valuation operator into a family of one-period pricing operators that can only be valuated in shorter intermediate time periods.

To derive the time-consistent actuarial value at the present time $t=0$, we divide the valuation period $[0, T]$ into a discrete set $\{0, \Delta t, 2 \Delta t, \ldots, T-\Delta t, T\}$ so that we can perform a multi-period valuation by applying the one-period pricing operator to all sub-intervals denoted by $(t, t+\Delta t)$. We use well-known actuarial premium principles such as the Variance, Standard-Deviation and Cost-of-Capital principles as pricing operators. Our aim is to apply the backward iteration method to all subintervals $(t, t+\Delta t) \in[0, T]$ to obtain the 
value of the related premium principle at time zero. We start with a payoff state space that is equal to the terminal values at time $T$ and calculate the one-period price at time $T-\Delta t$ for the last sub-interval $(T-\Delta t, T)$. This value space is derived by conditioning on the information available at $T-\Delta t$ and will look like a new payoff state space from the time $t-2 \Delta t$ viewpoint. Next, we repeat the one-period valuation process for the interval $(T-2 \Delta t . T-\Delta t)$. Conditional on the information available at $T-2 \Delta t$, we then obtain a new value state which plays the role of the new payoff state space for the former time period. The set of these conditional values can be used repeatedly as a new payoff state space for the former time points. We continue this backward valuation procedure for all subintervals of the form $(t, t+\Delta t)$ to gradually reach the time period $(0, \Delta t)$, where we derive the price of the actuarial risk at time zero.

The method is relatively straightforward and provides a discrete time valuation for the time-consistent actuarial premium principles. To derive the theoretical formulation of the time-consistent actuarial premium principle for a typical time interval $(t, t+\Delta t)$, we obtain the continuous-time limit of the premium operator at time $t$, on the premium value at time $t+\Delta t$ when $\Delta t \rightarrow 0$. This will lead to a PDE if the underlying insurance risk is a diffusion process and will lead to a PIDE if the underlying process has a jump component. The results can also be validated via a (bi/quadrinomial) discretization of the underlying process and by applying the same valuation method when $\Delta t \rightarrow 0$. In the applied situation, we achieve an approximation of the time-consistent premium by increasing the number of $(t, t+\Delta t)$ subintervals in $[0, T]$, which will decrease the size of $\Delta t$.

Let the mapping $\Pi_{t}: \mathscr{L}^{2}\left(\mathscr{F}_{T}\right) \rightarrow \mathscr{L}^{2}\left(\mathscr{F}_{t}\right)$ for $0 \leq t \leq T$ be the conditional one-period actuarial valuation operator (e.g. premium principle) with respect to $\mathscr{F}(t)$. We denote the price of the insurance risk (i.e. insurance premium) at time $t$ by $\pi(t, y(t))$. Then, $\pi(t, y(t))$ can be derived for any time interval $(t, t+\Delta t)$, by applying $\Pi_{t}$ to the payoff random variable at time $t+\Delta t$ denoted by $\pi(t+\Delta t, y(t, t+\Delta t))$ as below,

$$
\pi(t, y)=\Pi_{t}[\pi(t+\Delta t, y(t+\Delta t))]=\Pi\left[\pi(t+\Delta t, y(t+\Delta t)) \mid \mathscr{F}_{t}\right]
$$

In a backward iteration procedure, $\pi(t+\Delta t, y(t+\Delta t))$ is supposed to be the conditional value with respect to $\mathscr{F}_{t+\Delta t}$ obtained one step further from $\pi(t+2 \Delta t, y(t+2 \Delta t))$. We may also show " $y(t)$ " as " $y$ " later in some formulations to shorten the notation. For different products and liabilities, there may be possible boundary conditions. 


\subsection{Variance Pricing}

We start by considering an unhedgeable insurance process $y(t)$, which is given by means of a diffusion equation:

$$
d y(t)=a(t, y(t)) d t+b(t, y(t)) d W(t)
$$

We assume for $t \geq 0$, that $\mathscr{F}_{t}$ is the related filtration for $W_{t}$ and that $y(t)$ is an Itô process with $a(t, y(t))$ and $b(t, y(t))$ as adapted processes where $y(t)$ is still square integrable process.

Note that discounting is usually ignored in the standard actuarial literature (see for example Kaas et al., 2008). To facilitate the discussion, we will first derive the continuoustime limit of the Variance principle without using discounting in Section 2.2. We will then consider a case with discounting in Section 2.2, by means of a constant rate of discount for simplicity.

\section{Variance Principle}

If we consider an insurance contract with a payoff at time $T$, defined as a function $f(y(T))$, then the actuarial Variance principle $\Pi_{t}^{\mathrm{v}}[]$ is defined as (see e.g. Kaas et al., 2008)

$$
\Pi_{t}^{\mathrm{V}}[f(y(T))]=\mathbb{E}\left[f(y(T)) \mid \mathscr{F}_{t}\right]+\frac{1}{2} \alpha \operatorname{Var}\left[f(y(T)) \mid \mathscr{F}_{t}\right],
$$

where $\mathbb{E}_{t}\left[. \mid \mathscr{F}_{t}\right]$ and $\operatorname{Var}\left[. \mid \mathscr{F}_{t}\right]$ denote the expectation and variance operators conditional on the information available at time $t$ under the "real-world" probability measure $\mathbb{P}$. To keep the notation simple, we will use $\mathbb{E}_{t}[]$ and $\operatorname{Var}_{t}[]$ instead. The one-period Variance price can be obtained explicitly by substituting (2.2.2) into (2.1.3):

$$
\pi^{\mathrm{v}}(t, y(t))=\mathbb{E}_{t}\left[\pi^{\mathrm{v}}(t+\Delta t, y(t+\Delta t))\right]+\frac{1}{2} \alpha \operatorname{Var}_{t}\left[\pi^{\mathrm{V}}(t+\Delta t, y(t+\Delta t))\right]
$$

To calculate the continuous-time Variance price at (2.2.3), we could derive the stochastic process for $\pi^{\mathrm{v}}(t+\Delta t, y(t+\Delta t))$ and $\left(\pi^{\mathrm{v}}(t+\Delta t, y(t+\Delta t))\right)^{2}$ by Itô formula, divide the all terms by $\Delta t$ and take the limit when $\Delta t \rightarrow 0$. However a shorter proofs can be obtained by using the "infinitesimal generator" of the $\pi^{\mathrm{v}}$ and $\left(\pi^{\mathrm{v}}\right)^{2}$ at $t$. For the underlying process $y(t)$ in equation (4.2.1), the infinitesimal generator of $y(t)$ to act on the premium $\pi(t, y(t))$ is,

$$
\begin{aligned}
A \pi^{\mathrm{v}}(t, y(t)) & =\lim _{\Delta t \rightarrow 0} \frac{\mathbb{E}_{t}\left[\pi^{\mathrm{v}}(t+\Delta t, y(t+\Delta t))\right]-\pi^{\mathrm{v}}(t, y(t))}{\Delta t} \\
& =\pi_{t}^{\mathrm{v}}+a \pi_{y}^{\mathrm{v}}+\frac{1}{2} b^{2} \pi_{y y}^{\mathrm{v}},
\end{aligned}
$$

where $\pi^{\mathrm{V}}$ is smooth enough to be twice continuously differentiable at $t$ and $y=y(t)$. See, 
for example, Øksendal (2003) for more on infinitesimal generators. The short notations $\pi^{\mathrm{v}}$ and derivatives $\pi_{t}, \pi_{y}$ and $\pi_{y y}$ are continuous functions of $(t, y(t))$. To avoid too many

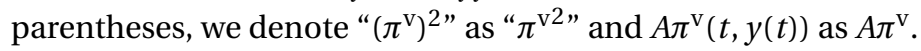

We rewrite the variance term in (2.2.3) by expectations and add and subtract $\pi^{\mathrm{v} 2}$ and $2 \pi^{\mathrm{v}} \mathbb{E}_{t}\left[\pi^{\mathrm{v}}\right]$ to obtain the equivalent expression

$\operatorname{Var}_{t}\left[\pi^{\mathrm{v}}(t+\Delta t)\right]=\mathbb{E}_{t}\left[\pi^{\mathrm{v} 2}(t+\Delta t)\right]-\pi^{\mathrm{v} 2}-\left(\mathbb{E}_{t}\left[\pi^{\mathrm{v}}(t+\Delta t)\right]-\pi^{\mathrm{v}}\right)^{2}-2 \pi^{\mathrm{v}}\left(\mathbb{E}_{t}\left[\pi^{\mathrm{v}}(t+\Delta t)\right]-\pi^{\mathrm{v}}\right)$,

where $\pi^{\mathrm{V}}(t+\Delta t)$ is a shorter notation of $\pi^{\mathrm{v}}(t+\Delta t, y(t+\Delta t))$. Dividing by $\Delta t$ and take the limit when $\Delta t \rightarrow 0$, the continuous-time limit of the variance term above will be

$$
\begin{aligned}
\lim _{\Delta t \rightarrow 0} \frac{\operatorname{Var}_{t}\left[\pi^{\mathrm{v}}(t+\Delta t)\right]}{\Delta t} & =A \pi^{\mathrm{v} 2}-\lim _{\Delta t \rightarrow 0} \Delta t \times\left(A \pi^{\mathrm{v}}\right)^{2}-2 \pi \times A \pi^{\mathrm{v}} \\
& =\left(b \pi_{y}^{\mathrm{v}}\right)^{2}
\end{aligned}
$$

where the first equality is justified by using (2.2.4a) while the limit term is clearly equal to zero, and the second equality is the result of substituting the values of infinitesimal generators from (2.2.4b) and some easy simplifications.

Finally, using (2.2.4) for expectation term in equation (2.2.3) and inserting for $A \pi^{\mathrm{v}}$ from (2.2.4b), we obtain the continuous-time limit of the Variance price represented by the following partial differential equation (PDE)

$$
\pi_{t}^{\mathrm{v}}+a \pi_{y}^{\mathrm{v}}+\frac{1}{2} b^{2} \pi_{y y}^{\mathrm{v}}+\frac{1}{2} \alpha\left(b \pi_{y}^{\mathrm{v}}\right)^{2}=0 .
$$

Note that due to the appearance of the quadratic term $\left(b \pi_{y}\right)^{2}$, equation (2.2.7) is a semilinear PDE. Assuming $\pi^{\mathrm{v}}(T, y(T))=f(y(T))$, as the payoff for the insurance contract at time $T$, depending on the mechanism of the different contracts, the PDE may be subject to different boundary conditions. We discussed a stylized contract in Section 3.2. Furthermore, the above PDE is equivalent to a Backward Stochastic Differential Equation (BSDE) with the quadratic driver $g(t, Z)=\frac{1}{2} \alpha(b Z)^{2}$. The existence of the solutions of BSDE has been investigated in numerous studies. See for example Delong (2013).

\section{Explicit Solution of the PDE}

In this particular case, we can construct the solution of (2.2.7) explicitly by employing a Hopf-Cole transformation of the solution that removes the non-linearity from the PDE. The result is only valid if $\alpha$ is a constant. Consider the auxiliary function $h^{\mathrm{v}}(t, y):=$ $\exp \left\{\alpha \pi^{\mathrm{V}}(t, y)\right\}$. The original function $\pi^{\mathrm{v}}(t, y)$ can be obtained from the inverse relation

$\pi^{\mathrm{v}}(t, y)=\frac{1}{\alpha} \ln h^{\mathrm{v}}(t, y)$. If we now apply the chain-rule of differentiation, we can express 
the partial derivatives of $\pi^{\mathrm{v}}()$ in terms of $h^{\mathrm{v}}()$ as

$$
\pi_{t}^{\mathrm{v}}=\frac{1}{\alpha} \frac{h_{t}^{\mathrm{v}}}{h^{\mathrm{v}}}, \quad \pi_{y}^{\mathrm{v}}=\frac{1}{\alpha} \frac{h_{y}^{\mathrm{v}}}{h^{\mathrm{v}}}, \quad \pi_{y y}^{\mathrm{v}}=\frac{1}{\alpha} \frac{h_{y y}^{\mathrm{v}} h^{\mathrm{v}}-\left(h_{y}^{\mathrm{v}}\right)^{2}}{\left(h^{\mathrm{v}}\right)^{2}} .
$$

If we substitute these expressions into (2.2.7), the non-linear terms are canceled and we obtain a linear PDE for $h^{\mathrm{v}}(t, y)$ :

$$
h_{t}^{\mathrm{v}}+a h_{y}^{\mathrm{v}}+\frac{1}{2} b^{2} h_{y y}^{\mathrm{v}}=0
$$

Hence, by considering the transformed function $h^{\mathrm{v}}(t, y)$, we have managed to obtain a linear PDE for $h^{\mathrm{v}}()$. The boundary condition at $T$ is given by $h^{\mathrm{v}}(T, y(T))=\exp \left\{\alpha \pi^{\mathrm{v}}(T, y(T))\right\}=$ $\exp \{\alpha f(y(T))\}$. Using the Feynman-Kaç formula, we can express the solution of (2.2.9) as

$$
h^{\mathrm{v}}(t, y)=\mathbb{E}_{t}\left[e^{\alpha f(y(T))} \mid y(t)=y\right],
$$

where the expectation is taken with respect to the stochastic process $y(t)$ defined in equation (4.2.1) conditional on the information that at time $t$ the process $y(t)$ is equal to $y$. From the representation (2.2.10), it immediately follows that we can express $\pi^{\mathrm{v}}(t, y)$ as

$$
\pi^{\mathrm{v}}(t, y)=\frac{1}{\alpha} \ln \mathbb{E}_{t}\left[e^{\alpha f(y(T))} \mid y(t)=y\right] .
$$

The form of the Variance price in the expectation part is equal to the moment generating function of the time $T$ payoff function $f(y(T))$, where for any known distribution of $f$ it will be easy to find a unique closed form formula for the premium. Also note that this representation of $\pi^{\mathrm{v}}()$ is identical to the exponential indifference price, which has been studied extensively in recent years. See, for example, Henderson (2002), Young and Zariphopoulou (2002), and Musiela and Zariphopoulou (2004). For an overview of recent advances in indifference pricing, we refer to the book by Carmona (2009).

To summarize this section, we have established that the continuous-time limit of the iterated actuarial Variance principle is the exponential indifference price when $\alpha$ is constant.

\section{Variance Pricing With Discounting}

Up to now we have ignored discounting in our derivation. (Or equivalently, we assumed that the interest rate is equal to zero.) In a time-consistent setting, it is important to take discounting into consideration, as money today cannot be compared to money tomorrow.

If we consider the definition of the Variance principle given in (2.2.2), it seems that we are adding apples and oranges. The first term $\mathbb{E}_{t}[f(y(T))]$ is a quantity in monetary units (say $€$ ) at time $T$. However, the second term $\operatorname{Var}_{t}[f(y(T))]$ is basically the expectation of 
$f(y(T))^{2}$, and is therefore a quantity in units of $(€)^{2}$. We can rectify this situation by understanding that the parameter $\alpha$ is not a dimensionless quantity, but is a quantity expressed in units of $1 / €$. This should not come as a surprise. The parameter $\alpha$ is similar to the absolute risk aversion parameter introduced by the seminal paper of Pratt (1964) in which he derives the Variance principle as an approximation "in the small" of the price that an economic agent facing a decision under uncertainty should ask.

To stress in our notation the units in which the absolute risk aversion $\alpha$ is expressed, we will rewrite the absolute risk aversion as the relative risk aversion $\gamma$ (also introduced by Pratt, 1964), which is a dimensionless quantity, divided by a benchmark wealth-level $X(T)$, which is expressed in $€$ at time $T$. If we now assume a constant rate of interest $r$, we can set our benchmark wealth as $X(T)=X_{0} e^{r T}$. We can then rewrite our Variance principle as

$$
\Pi_{t}^{\mathrm{V}}[f(y(T))]=\mathbb{E}_{t}[f(y(T))]+\frac{1}{2} \frac{\gamma}{X_{0} e^{r T}} \operatorname{Var}_{t}[f(y(T))] .
$$

Note that $\Pi_{t}^{\mathrm{v}}[]$ leads to a "forward" price expressed in units of $€$ at time $T$.

Given the enhanced definition (2.2.12) of the Variance principle including discounting, the one-period price will be delivered as follows:

$$
\pi^{\mathrm{v}}(t, y(t))=e^{-r \Delta t}\left(\mathbb{E}_{t}\left[\pi^{\mathrm{v}}(t+\Delta t, y(t+\Delta t))\right]+\frac{1}{2} \frac{\gamma}{X_{0} e^{r(t+\Delta t)}} \operatorname{Var}_{t}\left[\pi^{\mathrm{v}}(t+\Delta t, y(t+\Delta t))\right]\right)
$$

Note that we have included an additional discounting term $e^{-r \Delta t}$ to discount the values from time $t+\Delta t$ back to time $t$. We multiply both sides of (2.2.13) by $e^{r \Delta t}$ and use its Taylor series to obtain

$$
\left(1+r \Delta t+\mathscr{O}\left(\Delta t^{2}\right)\right) \pi^{\mathrm{v}}(t, y(t))=\mathbb{E}_{t}\left[\pi^{\mathrm{v}}(t+\Delta t, y(t+\Delta t))\right]+\frac{1}{2} \frac{\gamma}{X_{0} e^{r(t+\Delta t)}} \operatorname{Var}_{t}\left[\pi^{\mathrm{V}}(t+\Delta t, y(t+\Delta t))\right]
$$

Similar to the method in Section 2.2, if we divide by $\Delta t$ and take the limit, by (2.2.4a), the above equation can be represented as,

$$
r \pi^{\mathrm{v}}=A \pi^{\mathrm{v}}+\frac{1}{2} \frac{\gamma}{X_{0} e^{r t}}\left[A \pi^{\mathrm{v} 2}-2 \pi \times A \pi^{\mathrm{v}}\right] .
$$

The continuous-time limit of the time-consistent Variance price with discounting will be achievable easily by substituting for infinitesimal generators in above equation from (2.2.4b). That result in the following PDE for $\pi^{\mathrm{V}}(t, y)$ :

$$
\pi_{t}^{\mathrm{v}}+a \pi_{y}^{\mathrm{v}}+\frac{1}{2} b^{2} \pi_{y y}^{\mathrm{v}}+\frac{1}{2} \frac{\gamma}{X_{0} e^{r t}}\left(b \pi_{y}^{\mathrm{v}}\right)^{2}-r \pi^{\mathrm{v}}=0
$$

This non-linear PDE can again be linearized by considering $h^{\mathrm{v}}(t, y)=\exp \left(\frac{\gamma}{X_{0} e^{r t}} \pi^{\mathrm{v}}(t, y)\right)$ 
transformation, which leads to the following expression for the solution of (2.2.16):

$$
\pi^{\mathrm{v}}(t, y)=\frac{X_{0} e^{r t}}{\gamma} \ln \mathbb{E}\left[e^{\frac{\gamma}{X_{0} e^{r T}} f(y(T))} \mid y(t)=y\right] .
$$

This result shows that the discounting is incorporated into the non-linear pricing formula, by expressing all units relative to the "benchmark wealth" $X(t)=X_{0} e^{r t}$. See the chapter written by Musiela and Zariphopoulou (2009) in the book by Carmona (2009).

\section{Current price as benchmark}

In the previous subsection we took the benchmark wealth to be a risk-free investment $X_{0} e^{r t}$. Another interesting example can be found when we consider the current price $\pi(t, y)$ as the benchmark wealth. This leads to a new pricing operator, which we will denote by $\pi^{\mathrm{p}}()$. The one-step valuation is then given as

$$
\pi^{\mathrm{p}}(t, y(t))=e^{-r \Delta t}\left(\mathbb{E}_{t}\left[\pi^{\mathrm{p}}(t+\Delta t, y(t+\Delta t))\right]+\frac{1}{2} \gamma \frac{\operatorname{Var}_{t}\left[\pi^{\mathrm{p}}(t+\Delta t, y(t+\Delta t))\right]}{\mathbb{E}_{t}\left[\pi^{\mathrm{p}}(t+\Delta t, y(t+\Delta t))\right]}\right) .
$$

Hence, we assume that we want to measure the variance of $\pi^{\mathrm{p}}()$ relative to the expected value of $\pi^{\mathrm{p}}()$. Obviously, this will only be well-defined if $\pi^{\mathrm{p}}(t, y)$ is strictly positive for all $(t, y)$.

Taking the limit when $\Delta t \rightarrow 0$ in the above equation and applying the infinitesimal generator for $\pi^{P}$, we obtain the following PDE:

$$
\pi_{t}^{\mathrm{p}}+a \pi_{y}^{\mathrm{p}}+\frac{1}{2} b^{2} \pi_{y y}^{\mathrm{p}}+\frac{1}{2} \frac{\gamma}{\pi^{\mathrm{p}}}\left(b \pi_{y}^{\mathrm{p}}\right)^{2}-r \pi^{\mathrm{p}}=0
$$

Again, we can study the solution of (2.2.19) by employing a transformation of the solution that removes the non-linearity from the PDE. Consider the auxiliary function $h^{\mathrm{p}}(t, y):=$ $\left(\pi^{\mathrm{p}}(t, y)\right)^{1 / q}$. The original function can be obtained from the inverse relationship $\pi^{\mathrm{p}}(t, y)=$ $\left(h^{\mathrm{p}}(t, y)\right)^{q}$. If we now apply the chain rule, we can express the partial derivatives of $\pi^{\mathrm{p}}$ in terms of $h^{\mathrm{p}}$ as

$$
\pi_{t}^{\mathrm{p}}=q\left(h^{\mathrm{p}}\right)^{q-1} h_{t}^{\mathrm{p}}, \quad \pi_{y}^{\mathrm{p}}=q\left(h^{\mathrm{p}}\right)^{q-1} h_{y}^{\mathrm{p}}, \quad \pi_{y y}^{\mathrm{p}}=q\left(h^{\mathrm{p}}\right)^{q-1}\left(\frac{q-1}{h^{\mathrm{p}}}\left(h_{y}^{\mathrm{p}}\right)^{2}+h_{y y}^{\mathrm{p}}\right) .
$$

If we substitute these expressions into (2.2.19) and simplify, we obtain

$$
h_{t}^{\mathrm{p}}+a h_{y}^{\mathrm{p}}+\frac{1}{2} b^{2}\left(\frac{(1+\gamma) q-1}{h^{\mathrm{p}}}\left(h_{y}^{\mathrm{p}}\right)^{2}+h_{y y}^{\mathrm{p}}\right)-\frac{r}{q} h^{\mathrm{p}}=0 .
$$

\footnotetext{
${ }^{1}$ For general results concerning "benchmark pricing" in a linear setting, we refer to Platen (2006) and the book by Platen and Heath (2006).
} 
If we choose $q=1 /(1+\gamma)$, then the non-linear terms cancel out and we obtain a linear PDE for $h^{\mathrm{p}}(t, y)$ :

$$
h_{t}^{\mathrm{p}}+a h_{y}^{\mathrm{p}}+\frac{1}{2} b^{2} h_{y y}^{\mathrm{p}}-r(1+\gamma) h^{\mathrm{p}}=0 .
$$

The boundary condition at $T$ is given by $h^{\mathrm{p}}(T, y(T))=\pi^{\mathrm{p}}(T, y(T))^{1+\gamma}=f(y(T))^{1+\gamma}$. If we use the Feynman-Kaç formula, we can express the solution of (2.2.22) as

$$
h^{\mathrm{p}}(t, y)=\mathbb{E}_{t}\left[e^{-r(1+\gamma)(T-t)} f(y(T))^{1+\gamma} \mid y(t)=y\right],
$$

where the expectation is taken with respect to the stochastic process $y(t)$ defined in equation (4.2.1) conditional on the information that at time $t$ the process $y(t)$ is equal to $y$. From the representation (2.2.23), it immediately follows that we can express $\pi^{\mathrm{p}}(t, y)$ as

$$
\pi^{\mathrm{p}}(t, y)=e^{-r(T-t)}\left(\mathbb{E}_{t}\left[f(y(T))^{1+\gamma} \mid y(t)=y\right]\right)^{\frac{1}{1+\gamma}} .
$$

Note that this representation of the price $\pi^{\mathrm{p}}()$ also arises in the study of indifference pricing under power-utility functions, and the related notion of pricing under " $q$-optimal" measures. See, for example, Hobson (2004) and Henderson and Hobson (2009).

\section{Mean Value Principle}

The examples we gave in the previous subsections are all special cases of the Mean Value principle, which is defined as

$$
\Pi_{t}^{\mathrm{m}}[f(y(T))]=v^{-1}\left(\mathbb{E}_{t}[v(f(y(T)))]\right)
$$

for any convex and increasing function $v($ ) (see Kaas et al., 2008, Chap. 5).

Once more, we need to pay attention to units. If we want to apply a general function $v()$ to a value (expressed in units of $€$ ), we need to make sure that the argument of $v($ ) is dimensionless. The easiest way to achieve this is to express the argument for $v()$ in "forward terms". For a single time step of $(t, t+\Delta t)$, we therefore obtain the following expression for the price:

$$
\pi^{\mathrm{m}}(t, y(t))=v^{-1}\left(\mathbb{E}_{t}\left[v\left(e^{-r \Delta t} \pi^{\mathrm{m}}(t+\Delta t, y(t+\Delta t))\right)\right]\right)
$$

We can rewrite this definition as

$$
v\left(\frac{\pi^{\mathrm{m}}(t, y(t))}{e^{r t}}\right)=\mathbb{E}_{t}\left[v\left(\frac{\pi^{\mathrm{m}}(t+\Delta t, y(t+\Delta t))}{e^{r(t+\Delta t)}}\right)\right],
$$

from which it is immediately clear that the "distorted" value $v\left(\pi^{\mathrm{m}}(t, y) / e^{r t}\right)$ is linear and that it therefore satisfies the Feynman-Kaç formula. Therefore, its solution corresponds 
exactly to the solutions we found in the previous subsections.

As $v()$ is a Borel-measurable function, and if we assume $\mathbb{E}_{t} \mid v\left(\pi^{\mathrm{m}}(t, y) \mid<\infty\right.$, it becomes clear that the stochastic process $v\left(\pi^{\mathrm{m}}(t, y(t)) / e^{r t}\right)$ is a local martingale as the conditional expectation $\mathbb{E}_{t}[$.] is a martingale (see Shreve, 2010, Lemma 6.4.2). We can use this consideration to find the corresponding PDE for the price $\pi^{\mathrm{m}}(t, y)$. We can simplify this by defining the new process as $\pi^{\mathrm{mf}}(t, y):=\pi^{\mathrm{m}}(t, y) / e^{r t}$, which is the price expressed in forward terms. We use the Itô formula derivation for both stochastic processes $\pi^{\mathrm{mf}}(t, y)$, and also $v\left(\pi^{\mathrm{mf}}(t, y) / e^{r t}\right)$ with respect to $\pi^{\mathrm{mf}}(t, y)$. By applying the Itô formula to $\pi^{\mathrm{mf}}(t, y)$ with respect to (3.1), we get

$$
\pi^{\mathrm{mf}}(t, y)=\int_{0}^{t}\left(\pi_{t}^{\mathrm{mf}}+a \pi_{y}^{\mathrm{mf}}+\frac{1}{2} b^{2} \pi_{y y}^{\mathrm{mf}}\right) d s+\int_{0}^{t} b \pi_{y}^{\mathrm{mf}} d W(s),
$$

where we assumed $y(0)=\pi^{\mathrm{mf}}(0, y(0))=0$ at time $t=0$. Then, we apply the differential form of the Itô formula to function $v\left(\pi^{\mathrm{mf}}(t, y)\right)$ and using (2.2.28) we obtain,

$d v\left(\pi^{\mathrm{mf}}(t, y)\right)=\left[\left(\pi_{t}^{\mathrm{mf}}+a \pi_{y}^{\mathrm{mf}}+\frac{1}{2} b \pi_{y y}^{\mathrm{mf}}\right) v_{y}\left(\pi^{\mathrm{mf}}\right)+\frac{1}{2}\left(b \pi_{y}^{\mathrm{mf}}\right)^{2} v_{y y}\left(\pi^{\mathrm{mf}}\right)\right] d s+b \pi_{y}^{\mathrm{mf}} v_{y}\left(\pi^{\mathrm{mf}}\right) d W(s)$.

Note that considering $\pi^{\mathrm{mf}}$ a function of $(t, y), v()$ is no longer assumed to be a function of $t$ in Itô formula. Finally, as $v\left(\pi^{\mathrm{mf}}(t, y)\right)$ is a martingale process, the drift term can be set equal to zero:

$$
\left(\pi_{t}^{\mathrm{mf}}+a \pi_{y}^{\mathrm{mf}}+\frac{1}{2} b \pi_{y y}^{\mathrm{mf}}\right) v_{y}\left(\pi^{\mathrm{mf}}\right)+\frac{1}{2}\left(b \pi_{y}^{\mathrm{mf}}\right)^{2} v_{y y}\left(\pi^{\mathrm{mf}}\right)=0 .
$$

If we divide both sides by $v_{y}\left(\pi^{\mathrm{mf}}\right)$, we obtain the PDE for $\pi^{\mathrm{mf}}$,

$$
\pi_{t}^{\mathrm{mf}}+a \pi_{y}^{\mathrm{mf}}+\frac{1}{2} b \pi_{y y}^{\mathrm{mf}}+\frac{1}{2} \frac{v_{y y}\left(\pi^{\mathrm{mf}}\right)}{v_{y}\left(\pi^{\mathrm{mf}}\right)}\left(b \pi_{y}^{\mathrm{mf}}\right)^{2}=0,
$$

where this special derivation is true for any time step and we can relax the assumption of taking the limit when $\Delta t \rightarrow 0$. If we substitute the $\pi^{\mathrm{mf}}=e^{-r t} \pi^{\mathrm{m}}$ in (2.2.30) and simplify the notation, the corresponding PDE for the discounted Mean Value price will be

$$
\pi_{t}^{\mathrm{m}}+a \pi_{y}^{\mathrm{m}}+\frac{1}{2} b \pi_{y y}^{\mathrm{m}}+\frac{1}{2} \frac{v_{y y}\left(\pi^{\mathrm{m}}\right)}{v_{y}\left(\pi^{\mathrm{m}}\right)}\left(b \pi_{y}^{\mathrm{m}}\right)^{2}-r \pi^{\mathrm{m}}=0 .
$$

In both equations (2.2.30) and (2.2.31), we observe that the coefficient $v_{y y} / v_{y}$ in front of the non-linear term can be identified as the "local risk aversion", induced by the function $v()$ at the current value $\pi^{\mathrm{mf}}()$. Note that since the function $v()$ is increasing and convex by assumption, $v_{y y} / v_{y}$ is positive. Both forms of the PDE for the Mean Value principle are similar to the PDE of the Variance principle and have a quadratic driver for the equivalent BSDE in a time-consistent framework. 


\subsection{Standard-Deviation Pricing}

\section{Standard-Deviation Principle}

Another well-known actuarial pricing principle is the Standard-Deviation principle, defined as

$$
\Pi_{t}^{\mathrm{s}}[f(y(T))]=\mathbb{E}_{t}[f(y(T))]+\beta \sqrt{\operatorname{Var}_{t}[f(y(T))]}
$$

(see Kaas et al., 2008). Please note that in this case we also need to be careful about the dimensionality of the parameter $\beta$. Even though the expectation and the standard deviation are expressed in units of $€$, they both have different "time scales". If we use smaller time scales (as we will be doing when considering the limit for $\Delta t \rightarrow 0$ ) then, due to the diffusion term $d W$ of the process $y$, we have the property that the expectation of any function $f(y)$ scales linearly with $\Delta t$, but the standard deviation scales with $\sqrt{\Delta t}$. This means that the standard deviation term will literally overpower the expectation term for small $\Delta t$. Therefore, the only way to obtain a well-defined limit for $\Delta t \rightarrow 0$ is if we take $\beta \sqrt{\Delta t}$ as the parameter for the Standard-Deviation principle over the time step $(t, t+\Delta t)$.

Another way of understanding this result is to consider the following example. If we want to compare a standard deviation measured over an annual time step with a standard deviation measured over a monthly time step, we have to scale the annual outcome with $\sqrt{1 / 12}$ to get a fair comparison. Given the above discussion on dimensionality and the time scales, we will then get the following expression for the one-step price:

$$
\pi^{\mathrm{s}}(t, y(t))=e^{-r \Delta t}\left(\mathbb{E}_{t}\left[\pi^{\mathrm{s}}(t+\Delta t, y(t+\Delta t))\right]+\beta \sqrt{\Delta t} \sqrt{\operatorname{Var}_{t}\left[\pi^{\mathrm{s}}(t+\Delta t, y(t+\Delta t))\right]}\right) .
$$

We multiply both sides by $e^{r \Delta t}$, use its Taylor expansion, divide by $\Delta t$ and take the limit. With some simplifications we obtain,

$$
\begin{aligned}
r \pi^{\mathrm{s}}(t, y(t)) & =\lim _{\Delta t \rightarrow 0} \frac{\mathbb{E}_{t}\left[\pi^{\mathrm{s}}(t+\Delta t, y(t+\Delta t))\right]-\pi^{\mathrm{s}}(t, y(t))}{\Delta t}+\beta \sqrt{\lim _{\Delta t \rightarrow 0} \frac{\operatorname{Var}_{t}\left[\pi^{\mathrm{s}}(t+\Delta t, y(t+\Delta t))\right]}{\Delta t}} \\
& =\pi_{t}^{\mathrm{s}}+a \pi_{y}^{\mathrm{s}}+\frac{1}{2} b^{2} \pi_{y y}^{\mathrm{s}}+\beta \sqrt{\left(b \pi_{y}^{\mathrm{s}}\right)^{2}}
\end{aligned}
$$

where in the second equality we used the definition of the infinitesimal generator in (2.2.4) for expectation term and equation (2.2.6) for variance term. Hence, we arrive at the following partial differential equation for $\pi^{\mathrm{s}}(t, y(t))$ :

$$
\pi_{t}^{\mathrm{s}}+a \pi_{y}^{\mathrm{s}}+\frac{1}{2} b^{2} \pi_{y y}^{\mathrm{s}}+\beta b\left|\pi_{y}^{\mathrm{s}}\right|-r \pi^{\mathrm{s}}=0
$$

This is again a semi-linear PDE that can be represented by a BSDE with a Lipschitz driver, 
$g(t, Z)=\beta|b Z|$. However, the semi-linearity is much more benign in this case. Whenever the sign of the partial derivative $\pi_{y}^{\mathrm{s}}$ does not change anywhere in the domain of $y$ (i.e. the function $\pi^{\mathrm{s}}$ either monotonically increases or monotonically decreases in $y$ ), then (2.3.4) is reduced to the linear PDE:

$$
\pi_{t}^{\mathrm{s}}+(a \pm \beta b) \pi_{y}^{\mathrm{s}}+\frac{1}{2} b^{2} \pi_{y y}^{\mathrm{s}}-r \pi^{\mathrm{s}}=0
$$

where the sign of $\pm \beta b$ depends on the (uniquely defined) sign of $\pi_{y}^{\mathrm{s}}$.

Using the Feynman-Kaç formula, we can represent the solution of (2.3.5) as follows:

$$
\pi^{\mathrm{s}}(t, y)=\mathbb{E}_{t}^{\mathbb{S}}\left[e^{-r(T-t)} f(y(T)) \mid y(t)=y\right],
$$

where $\mathbb{E}_{t}^{\mathbb{S}}$ [] denotes the expectation at time $t$ with respect to the "risk-adjusted" process $y^{\mathbb{S}}$ defined as

$$
d y^{\mathbb{S}}=(a(t, y) \pm \beta b(t, y)) d t+b(t, y) d W^{\mathbb{S}} .
$$

The risk-adjusted process is consistent with the concept of actuarial prudence, where the insurer calculates the premium using an adjusted drift to make a more conservative assessment of expectation. Mathematically, the drift rate is adjusted upwards $(a+\beta b)$ if the payoff $f(y)$ monotonically increases in $y$, and is adjusted downwards $(a-\beta b)$ if $f(y)$ monotonically decreases in $y$. So, the risk adjustment is always in the "upwind" direction of the risk, making the price $\pi^{\mathrm{s}}$ more expensive than the real-world expectation $\mathbb{E}[f(y)]$.

\section{Cost-of-Capital Principle}

Another actuarial pricing principle is the Cost-of-Capital principle. The Cost-of-Capital method has been widely adopted by the insurance industry in Europe, and has also been prescribed as the standard method by the European Insurance and Pensions Supervisor for the Quantitative Impact Studies (see EIOPA, 2010). ${ }^{2}$

The Cost-of-Capital principle is based on the following economic reasoning. We first consider the "expected loss" $\mathbb{E}[f(y(T)]$ of the insurance claim $f(y(T))$ as a basis for pricing. In addition, the insurance company needs to hold a capital buffer against the "unexpected loss". This buffer is calculated as a Value-at-Risk (VaR) over a time horizon (typically 1 year) and a probability threshold $q$ (usually 0.995 for insurance). The unexpected loss is then calculated as $\operatorname{VaR}_{q}[f(y(T))-\mathbb{E}[f(y(T))]] .{ }^{3}$ The capital buffer is borrowed from the shareholders of the insurance company; however, there is a small probability $(1-q)$ that

\footnotetext{
${ }^{2}$ The idea of valuation based on the cost of capital, was introduced by the Swiss insurance supervisor as a part of the method used to calculate solvency capitals for insurance companies (Keller and Luder, 2004). For a critical discussion on the risk measure implied by the Swiss Solvency Test, we refer to Filipovic and Vogelpoth (2008).

${ }^{3}$ Although using VaR is in line with Solvency II and EIOPA directives, the Swiss insurance supervisor used "Expected shortfall" (also called "conditional value at risk (CVaR)" or "average value at risk (AVaR)") instead of VaR.
} 
the capital buffer is needed to cover an unexpected loss. Hence, the shareholders require a compensation for this risk in the form of a "cost of capital". This cost of capital needs to be included in the pricing of the insurance contract. If we denote the cost of capital by $\delta$, then the Cost-of-Capital principle is given by

$$
\Pi_{t}^{\mathrm{c}}[f(y(T))]=\mathbb{E}_{t}[f(y(T))]+\delta \operatorname{VaR}_{q, t}\left[f(y(T))-\mathbb{E}_{t}[f(y(T))]\right] .
$$

Note that, we also need to be careful about the dimensionality of the different terms in this case. First, we are comparing VaR quantities at different time scales, and these have to be scaled back to a per-annum basis. To do this we divide the VaR term by $\sqrt{\Delta t}$. We must then realize that the cost of capital $\delta$ behaves like an interest rate: it is the compensation the insurance company needs to pay its shareholders for borrowing the buffer capital over a certain period. The cost of capital is expressed as a percentage per annum; hence over a time-step $\Delta t$ the insurance company will have to pay a compensation of $\delta \Delta t$ per $€$ of buffer capital. As a result, we obtain a "net scaling" of $\delta \Delta t / \sqrt{\Delta t}=\delta \sqrt{\Delta t}$. Note that this is the same scaling as for the Standard-Deviation principle. For a single time-step, we therefore get the following expression for the Cost-of-Capital price:

$$
\begin{aligned}
\pi^{\mathrm{c}}(t, y(t))=e^{-r \Delta t}\left(\mathbb{E}_{t}\left[\pi^{\mathrm{c}}(t+\Delta t, y(t+\Delta t))\right]\right. & \\
& \left.+\delta \sqrt{\Delta t} \operatorname{VaR}_{q, t}\left[\pi^{\mathrm{c}}(t+\Delta t, y(t+\Delta t))-\mathbb{E}_{t}\left[\pi^{\mathrm{c}}(t+\Delta t, y(t+\Delta t))\right]\right]\right) .
\end{aligned}
$$

Applying the method that we used in Subsections 2.2 and 2.3, the continuous-time limit of the above equation is

$r \pi^{\mathrm{c}}(t, y(t))=A \pi^{\mathrm{c}}\left(t, y(t)+\delta \sqrt{\Delta t} \operatorname{VaR}_{q, t}\left[\lim _{\Delta t \rightarrow 0} \frac{\pi^{\mathrm{c}}(t+\Delta t, y(t+\Delta t))-\mathbb{E}_{t}\left[\pi^{\mathrm{c}}(t+\Delta t, y(t+\Delta t))\right]}{\Delta t}\right]\right.$.

Using the integral form of the Itô formula for $\pi^{\mathrm{c}}(t+\Delta t, y(t+\Delta t))$, the expression under the limit in VaR operator can be written as

$$
\begin{aligned}
& \lim _{\Delta t \rightarrow 0} \frac{\pi^{\mathrm{c}}(t, y(t))-\mathbb{E}_{t}\left[\pi^{\mathrm{c}}(t+\Delta t, y(t+\Delta t))\right]}{\Delta t} \\
&+\lim _{\Delta t \rightarrow 0} \frac{1}{\Delta t} \int_{t}^{t+\Delta t}\left(\pi_{t}^{\mathrm{c}}(s, y(s))+a \pi_{y}^{\mathrm{c}}(s, y(s))+\frac{1}{2} b^{2} \pi_{y y}^{\mathrm{c}}(s, y(s))\right) d s \\
&+\lim _{\Delta t \rightarrow 0} \frac{1}{\Delta t} \int_{t}^{t+\Delta t} b \pi_{y}^{\mathrm{c}}(s, y(s)) d W(s)
\end{aligned}
$$

The first limit by equation (2.2.4) is equal to $-\left(\pi_{t}^{\mathrm{c}}(t, y(t))+a \pi_{y}^{\mathrm{c}}(t, y(t))+\frac{1}{2} b^{2} \pi_{y y}^{\mathrm{c}}(t, y(t))\right)$. If we assume $f(s, y(s))=\pi_{t}^{\mathrm{c}}(s, y(s))+a \pi_{y}^{\mathrm{c}}(s, y(s))+\frac{1}{2} b^{2} \pi_{y y}^{\mathrm{c}}(s, y(s))$ is a continuous differen- 
tiable function, by definition of the limit for such a function, the second term will be

$$
\begin{aligned}
& \lim _{\Delta t \rightarrow 0} \frac{1}{\Delta t} \int_{t}^{t+\Delta t}\left(\pi_{t}^{\mathrm{c}}(s, y(s))+a \pi_{y}^{\mathrm{c}}(s, y(s))+\frac{1}{2} b^{2} \pi_{y y}^{\mathrm{c}}(s, y(s))\right) d s \\
&=\pi_{t}^{\mathrm{c}}(t, y(t))+a \pi_{y}^{\mathrm{c}}(t, y(t))+\frac{1}{2} b^{2} \pi_{y y}^{\mathrm{c}}(t, y(t))
\end{aligned}
$$

where we recall that $a$ and $b$, the drift and diffusion rates under the integration, are also functions of $s$ and $y(s)$ for $s>t$. This cancels the first and the second terms of equation (2.3.11) and leaves the third term, which is an Itô integral, to be valuated.

Valuation of the Itô integral under the $\mathrm{VaR}_{1-q, t}$ function is a critical part of this premium. We denote this integral as,

$$
Z(t+\Delta t)=Z(t)+\int_{t}^{t+\Delta t} b(s, y(s)) \pi_{y}^{\mathrm{c}}(s, y(s)) d W(s) .
$$

In general, the integrand $b(s, y(s)) \pi^{\mathrm{c}}(s, y(s))$ in (2.3.13) for $s>t$ is an adapted stochastic process. In this situation, it is difficult to draw inferences about the distribution of the above Itô integral and to give a more direct calculation for $\operatorname{VaR}_{q, t}$. Although we do not know the analytical distribution of $Z(t+\Delta t)$, we can obtain its first two moments with respect to the filtration $\mathscr{F}_{t}$. As the Itô integral is a martingale, its conditional expectation with respect to the filtration $\mathscr{F}_{t}$ is zero,

$$
\mathbb{E}\left[\int_{t}^{t+\Delta t} b(s, y(s)) \pi_{y}^{\mathrm{c}}(s, y(s)) d W(s) \mid \mathscr{F}_{t}\right]=0,
$$

where its variance can be obtained based on the Itô isometry for stochastic integrands as follows:

$$
\begin{aligned}
\operatorname{Var}\left[\int_{t}^{t+\Delta t} b(s, y(s)) \pi_{y}^{\mathrm{c}}(s, y(s)) d W(s) \mid \mathscr{F}_{t}\right] & =\mathbb{E}_{t}\left[\left(\int_{t}^{t+\Delta t} b(s, y(s)) \pi_{y}^{\mathrm{c}}(s, y(s)) d W(s)\right)^{2}\right] \\
& =\int_{t}^{t+\Delta t} \mathbb{E}_{t}\left[\left(b(s, y(s)) \pi_{y}^{\mathrm{c}}(s, y(s))\right)^{2}\right] d s .
\end{aligned}
$$

Since we want to compute the continuous-time limit of the price in an EulerMaruyama approximation setting when $\Delta t \rightarrow 0$, we assume $Z(t+\Delta t)-Z(t)$ as a partition $(t, t+\Delta t)$ of the process $Z$ with drift zero in $[0, T]$. Kloeden and Platen (1999) have discussed the Euler-Maruyama discretization of the stochastic processes. Using the weak convergence of this approximation, when $\Delta t \rightarrow 0$ we have

$$
\frac{1}{\Delta t} \int_{t}^{t+\Delta t} b(s, y(s)) \pi_{y}^{\mathrm{c}}(s, y(s)) d W(s) \approx \frac{1}{\Delta t}\left[b(t, y(t)) \pi_{y}^{\mathrm{c}}(t, y(t)) \Delta W(t)\right],
$$


where $\Delta W(t)=W(t+\Delta t)-W(t)$ is an independent and identically distributed normal random variable with expected value zero and variance $\Delta t$ for all $0<t \leq T$. Note that at time $t, b(t, y(t)) \pi_{y}^{\mathrm{c}}(t, y(t))$ is non-random and when $\Delta t$ is extremely small, the distribution of $Z(t+\Delta t)$ is approximately normal. By this assumption, we can conclude that when $\Delta t \rightarrow 0$, the approximated term is normally distributed

$$
\frac{1}{\Delta t} \int_{t}^{t+\Delta t} b(s, y(s)) \pi_{y}^{\mathrm{c}}(s, y(s)) d W(s) \sim N\left(0, \frac{\left(b(t, y(t)) \pi_{y}^{\mathrm{c}}(t, y(t))\right)^{2}}{\Delta t}\right) .
$$

This also shows that in (2.3.15) when $\Delta t \rightarrow 0$,

$$
\frac{1}{\Delta t} \operatorname{Var}\left[\int_{t}^{t+\Delta t} b(s, y(s)) \pi_{y}^{\mathrm{c}}(s, y(s)) d W(s) \mid \mathscr{F}_{t}\right] \approx\left(b \pi_{y}^{\mathrm{c}}\right)^{2} .
$$

Using "translation and scaling invariance" property of the VaR function with respect to a non-negative constant, we have:

$$
\lim _{\Delta t \rightarrow 0} \operatorname{VaR}_{q, t}\left[\frac{1}{\Delta t} \int_{t}^{t+\Delta t} b(s, y(s)) \pi_{y}^{\mathrm{c}}(s, y(s)) d W(s)\right]=\frac{1}{\sqrt{\Delta t}} b(t, y(t))\left|\pi_{y}^{\mathrm{c}}(t, y(t))\right| \Phi^{-1}(q) .
$$

Finally, recalling equation (2.3.10) and inserting the limit above instead of the VaR limit term and so for $A \pi^{\mathrm{c}}$ from (2.2.4b), we derive the related PDE for the Cost-of-Capital premium principle as

$$
\pi_{t}^{\mathrm{c}}+a \pi_{y}^{\mathrm{c}}+\frac{1}{2} b^{2} \pi_{y y}^{\mathrm{c}}+\delta k b\left|\pi_{y}^{\mathrm{c}}\right|-r \pi^{\mathrm{c}}=0 .
$$

where $k=\Phi^{-1}(q)$. This PDE is the same as the one we obtained in (2.3.4) for the StandardDeviation price, except for the factor $\delta k$, which replaces $\beta$ in front of $b\left|\pi_{y}^{\mathrm{c}}\right|$. This should not come as a surprise, since the $(q)$-quantile of $y(t+\Delta t)$ for a small time-step $\Delta t$ converges to $k$ times the standard deviation $b \sqrt{\Delta t}$, and hence the Cost-of-Capital pricing operator $\pi^{\mathrm{c}}()$ should converge to the Standard-Deviation pricing operator $\pi^{\mathrm{s}}()$ with $\beta=\delta k$.

If the payoff $f(y(T))$ is monotonous in $y(T)$, we can represent the Cost-of-Capital price $\pi^{\mathrm{c}}(t, y)$ in the same way as the Standard-Deviation price (2.3.6) with respect to the riskadjusted process $y$ :

$$
d y=(a(t, y) \pm \delta k b(t, y)) d t+b(t, y) d W
$$





\section{3}

Time-Consistent Actuarial Valuations under Jump-Diffusion Processes

\section{This chapter is based on:}

Pelsser, A. and Salahnejhad Ghalehjooghi, A. (2016), Time-consistent Actuarial Valuation, Insurance Mathematics and Economics, Vol 66, pp:97-112.

Heavy-tailed distribution is a stylized attribute for the majority of the insurance liabilities. This heavy-tailed nature is a motivation to model the actuarial risk under the jumpdiffusion process to get a more realistic picture of the underlying risk dynamics. In this chapter, we extend the concept of time-consistent actuarial pricing by adding a jump component to the valuation process. In fact, we generalize the backward iteration of the oneperiod valuation of the insurance premium principles when the unhedgeable insurance process can also jump by an stochastic arrival time. Furthermore, we no longer observe that the different premium principles converge to the same limit since each principle reflects the effect of the jump differently. In the Cost-of-Capital principle, in particular the VaR operator fails to capture the jump risk for small jump probabilities, and the timeconsistent price depends on the distribution of the premium jump. 


\subsection{Time-consistent Pricing under Jump Process}

Let $\left(\Omega, \mathscr{F}_{t}, \mathbb{P}\right) \quad t \geq 0$ be the filtered probability space. We use the model of Merton (1976) where the insurance process $y(t)$ follows the jump process of the form

$$
d y(t)=a(t, y(t)) d t+b(t, y(t)) d W(t)+C\left(t_{-}, y\left(t_{-}\right)\right) d N(t)
$$

where $C\left(t_{-}, y\left(t_{-}\right)\right)=y(t)-y\left(t_{-}\right)$(with shorter notation " $C(t)$ ”) is the bounded jump size random variable with $\mathbb{E}[C(t)]=\beta$, and $N(t)$ be the Poisson counting process of the jumps with conditional intensity $\lambda(t, y(t))$ where $\lambda$ is a continuous function and $N(0)=0$. Note that, $y\left(t_{-}\right)$is the left continuous version of $y(t)$. We assume we have finitely many jumps in any finite time interval of the form $\left(t, t+\Delta t\right.$ ]. Moreover, $W(t), N(t)$ and $C(t)$ are $\mathscr{F}_{t^{-}}$ measurable processes with independent increment. Note that $N(t)$ and $C(t)$ are assumed to be independent while together they form a compound Poisson process which is also $\mathscr{F}_{t}$-measurable with independent increment.

\section{Variance Pricing with Jump}

In this section we directly apply the case of Variance pricing with discounting and we employ the one-period valuation of this premium principle to obtain a time-consistent price. We recall (2.2.13) as the main pricing rule,

$$
\pi^{\mathrm{v}}(t, y(t))=e^{-r \Delta t}\left(\mathbb{E}_{t}\left[\pi^{\mathrm{v}}(t+\Delta t, y(t+\Delta t))\right]+\frac{1}{2} \frac{\gamma}{X_{0} e^{r(t+\Delta t)}} \operatorname{Var}_{t}\left[\pi^{\mathrm{v}}(t+\Delta t, y(t+\Delta t))\right]\right)
$$

where $\pi^{\mathrm{v}}(t, y(t))$ is a sufficiently smooth function and twice continuously differentiable with respect to both $y$ and $t$. We recall equation (2.2.15) as the continuous-time limit of the variance price in terms of the infinitesimal generator

$$
r \pi^{\mathrm{v}}=A \pi^{\mathrm{v}}+\frac{1}{2} \frac{\gamma}{X_{0} e^{r t}}\left[A \pi^{\mathrm{v} 2}-2 \pi^{\mathrm{v}} \times A \pi^{\mathrm{v}}\right]
$$

The infinitesimal generator for $\pi$ with above conditions is defined in (2.2.4a) where for a $y(t)$ modeled by (3.1.1) at $(t, y(t))$ it has a different form as below,

$$
A_{J} \pi^{\mathrm{v}}(t, y(t))=\pi_{t}^{\mathrm{v}}+a \pi_{y}^{\mathrm{v}}+\frac{1}{2} b^{2} \pi_{y y}^{\mathrm{v}}+\lambda(t, y(t)) \mathbb{E}\left[\pi^{\mathrm{v}}(t, y(t)+C(t))-\pi^{\mathrm{v}}(t, y(t))\right]
$$

where the subscript " $J$ " in $A_{J}$ exhibits the jump version of the infinitesimal generator and $\pi^{\mathrm{v}}(t, y(t)+C(t))-\pi^{\mathrm{v}}(t, y(t))$ is the possible premium jump at time $t$. For the sake of clarity, we should mention again that the derivative terms are functions of $(t, y(t))$ which is suppressed to shorten the notation. For more on the infinitesimal generators of the jump processes, see for example, Applebaum (2004). 
In general, the expression $A_{J} \pi^{\mathrm{v} 2}-2 \pi \times A_{J} \pi^{\mathrm{v}}$ still, by definition, represents the limit of the variance term (See the first equality of equation (2.2.6)). once again we remind that $\pi^{\mathrm{v} 2}$ is the shorter notation for square of $\pi^{\mathrm{v}}$. We calculate the alternative form of the above expression for the jump-diffusion process. By (3.1.2) and using the chain rule for derivatives of $\pi^{\mathrm{v} 2}$, we have

$A_{J} \pi^{\mathrm{v} 2}=2 \pi^{\mathrm{v}}\left[\pi_{t}^{\mathrm{v}}+a \pi_{y}^{\mathrm{v}}+\frac{1}{2} b^{2} \pi_{y y}^{\mathrm{v}}\right]+\frac{1}{2} b^{2}\left(\pi_{y}^{\mathrm{v}}\right)^{2}+\lambda(t, y(t)) \mathbb{E}\left[\pi^{\mathrm{v} 2}(t, y(t)+C(t))-\pi^{\mathrm{v} 2}(t, y(t))\right]$

and

$$
2 \pi^{\mathrm{v}} A_{J} \pi^{\mathrm{v}}=2 \pi^{\mathrm{v}}\left[\pi_{t}^{\mathrm{v}}+a \pi_{y}^{\mathrm{v}}+\frac{1}{2} b^{2} \pi_{y y}^{\mathrm{v}}\right]+\lambda(t, y(t)) \mathbb{E}\left[2 \pi^{\mathrm{v}}(t, y(t)) \pi^{\mathrm{v}}(t, y(t)+C(t))-2 \pi^{\mathrm{v} 2}(t, y(t))\right] .
$$

Hence, the limit of the variance term is

$$
\begin{aligned}
\lim _{\Delta t \rightarrow 0} \operatorname{Var}\left[\pi^{\mathrm{v}}(t+\Delta t, y(t+\Delta t))\right] & =A_{J} \pi^{\mathrm{v} 2}-2 \pi \times A_{J} \pi^{\mathrm{v}} \\
& =\frac{1}{2} b^{2}\left(\pi_{y}^{\mathrm{v}}\right)^{2}+\lambda(t, y(t)) \mathbb{E}\left[\left(\pi^{\mathrm{v}}(t, y(t)+C(t))-\pi^{\mathrm{v}}(t, y(t))\right)^{2}\right] .
\end{aligned}
$$

Finally, inserting for $A_{J} \pi^{\mathrm{v}}$ and $A_{J} \pi^{\mathrm{v} 2}-2 \pi \times A_{J} \pi^{\mathrm{v}}$ into (2.2.15), respectively from (3.1.2) and (3.1.5), we obtain the new form of the differential equation for Variance pricing including a jump component:

$$
\begin{array}{r}
\pi_{t}^{\mathrm{v}}+a \pi_{y}^{\mathrm{v}}+\frac{1}{2} b^{2} \pi_{y y}^{\mathrm{v}}+\frac{1}{2} \frac{\gamma}{X_{0} e^{r t}}\left(b \pi_{y}^{\mathrm{v}}\right)^{2}-r \pi^{\mathrm{v}}+\lambda(t, y(t))\left[\mathbb{E}\left[\pi^{\mathrm{v}}(t, y(t)+C(t))-\pi^{\mathrm{v}}(t, y(t))\right]\right. \\
\left.+\frac{1}{2} \frac{\gamma}{X_{0} e^{r t}} \mathbb{E}\left[\left(\pi^{\mathrm{v}}(t, y(t)+C(t))-\pi^{\mathrm{v}}(t, y(t))\right)^{2}\right]\right]=0,
\end{array}
$$

where $\lambda \mathbb{E}\left[\left(\pi^{\mathrm{v}}(t, y(t)+C(t))-\pi^{\mathrm{v}}(t, y(t))\right)^{2}\right]$ can be interpreted as the instantaneous variance of the compound Poisson jump for the premium at time $t$. Considering $y(t)$ as a special Lévy process with the jump size random variable $C(t)$ and the Lévy measure $v(d c)$, we can exhibit (3.1.6) by a more standard formulation,

$$
\begin{aligned}
& \pi_{t}^{\mathrm{V}}+a \pi_{y}^{\mathrm{v}}+\frac{1}{2} b^{2} \pi_{y y}^{\mathrm{v}}+\frac{1}{2} \frac{\gamma}{X_{0} e^{r t}}\left(b \pi_{y}^{\mathrm{v}}\right)^{2}-r \pi^{\mathrm{v}} \\
&+\lambda(t, y(t)) \int\left(\pi^{\mathrm{v}}(t, y(t)+c)-\pi^{\mathrm{v}}(t, y(t))+\frac{1}{2} \frac{\gamma}{X_{0} e^{r t}}\left(\pi^{\mathrm{v}}(t, y(t)+c)-\pi^{\mathrm{v}}(t, y(t))\right)^{2}\right) v(d c)=0 .
\end{aligned}
$$

The above equation is a Partial Integro-Differential Equation (PIDE), as the expectation terms can be rephrased in the form of integrals of the premium jump on the jump size in the related sample space. (3.1.7) is a semi-linear PIDE where it includes quadratic terms 
of both continuous and jump components. The quadratic term again represents that the equivalent BSDE for this PIDE will have a quadratic driver $g(t, Z)=\frac{1}{2} \frac{\gamma}{X_{0} e^{r t}}(b Z)^{2}$. It also includes the probability of one jump for any point at time $t>0$ by means of the parameter $\lambda$. Conditional on a "one-jump" event, the integral (expectation) terms then formulate the effect of the jump size on the value of $\pi^{\mathrm{v}}(t, y)$. It is also clear that the PDE in (2.2.16) is a special case of the (3.1.7) PIDE where there is no jump in the insurance process.

\section{Mean-Value Price with Jump}

In the previous case we assumed a simple jump-diffusion process (3.1.1) to drive the underlying risk process $y(t)$ and we obtained the proper PIDE to describe the time-consistent Variance premium principle with a jump. Again, to find the PIDE for the Mean Value principle in the jump case, we need to reform the equation (2.2.27) as the pricing rule. To do so, we still need the martingale property for $\nu\left(\frac{\pi^{\mathrm{m}}(t, y(t))}{e^{r t}}\right)$, where $\frac{\pi^{\mathrm{m}}(t, y(t))}{e^{r t}}=\pi^{\mathrm{mf}}(t, y(t))$. The implicit compound Poisson process to describe the jumps in (3.1.1) is not enough to achieve the martingale property for $\pi^{\mathrm{mf}}(t, y(t))$. Instead we use the compensated version of the Poisson process in (3.1.1) as below,

$$
d y(t)=\left[a(t, y(t))+\lambda(t, y(t)) C\left(t_{-}, y\left(t_{-}\right)\right)\right] d t+b(t, y(t)) d W(t)+C\left(t_{-}, y\left(t_{-}\right)\right) d \tilde{N}(t), \quad(3.1 .8)
$$

where $\tilde{N}(t)=N(t)-\lambda(t, y(t)) \times t$ is the compensated Poisson process. As we need to evaluate $v\left(\pi^{\mathrm{mf}}(t, y(t))\right)$, we can apply the Itô formula in two steps for $\pi^{\mathrm{mf}}(t, y(t))$ with respect to $t$ and $y(t)$ and then for $v\left(\pi^{\mathrm{mf}}\right)$ with respect to $\pi^{\mathrm{mf}}$. The resulted stochastic processes for $\pi^{\mathrm{mf}}$ is

$$
\begin{array}{r}
\pi^{\mathrm{mf}}(t, y)=\left(\pi_{t}^{\mathrm{mf}}+\lambda \mathbb{E}\left[\pi^{\mathrm{mf}}(t, y(t)+C(t))-\pi^{\mathrm{mf}}(t, y(t))\right]+a \pi_{y}^{\mathrm{mf}}+\frac{1}{2} b^{2} \pi_{y y}^{\mathrm{mf}}\right) d s+b \pi_{y}^{\mathrm{mf}} d W(t) \\
+\left(\pi^{\mathrm{mf}}(t, y(t)+C(t))-\pi^{\mathrm{mf}}(t, y(t))\right) d \tilde{N}(t)
\end{array}
$$

where $\lambda$ is shorter notation of $\lambda(t, y(t))$. Similarly for $v\left(\pi^{\mathrm{mf}}\right)$ we have,

$$
\begin{array}{r}
d v\left(\pi^{\mathrm{mf}}(t, y)\right)=\left\{\left[\pi_{t}^{\mathrm{mf}}+\lambda \mathbb{E}\left[\pi^{\mathrm{mf}}(t, y(t)+C(t))-\pi^{\mathrm{mf}}(t, y(t))\right]+a \pi_{y}^{\mathrm{mf}}+\frac{1}{2} b \pi_{y y}^{\mathrm{mf}}\right] v_{y}\left(\pi^{\mathrm{mf}}\right)\right. \\
\left.+\frac{1}{2}\left(b \pi_{y}^{\mathrm{mf}}\right)^{2} v_{y y}\left(\pi^{\mathrm{mf}}\right)+\lambda \mathbb{E}\left[v\left(\pi^{\mathrm{mf}}(t, y(t)+C(t))\right)-v\left(\pi^{\mathrm{mf}}(t, y(t))\right)\right]\right\} d t+b \pi_{y}^{\mathrm{mf}} v_{y}\left(\pi^{\mathrm{mf}}\right) d W(t) \\
+\left[v\left(\pi^{\mathrm{mf}}(t, y(t)+C(t))\right)-v\left(\pi^{\mathrm{mf}}(t, y(t))\right)\right] d \tilde{N}(t) .
\end{array}
$$

According to (2.2.27) and the martingale property of $\mathbb{E}_{t}\left[v\left(\pi^{\mathrm{mf}}(t+\Delta t, y(t+\Delta t))\right)\right]$, the compensated Poisson jump process of $v\left(\pi^{\mathrm{mf}}(t, y(t))\right)$ in (3.1.10) should also be martin- 
gale. So, we set the drift term above equal to zero:

$$
\begin{aligned}
& {\left[\pi_{t}^{\mathrm{mf}}+a \pi_{y}^{\mathrm{mf}}+\frac{1}{2} b \pi_{y y}^{\mathrm{mf}}+\lambda \mathbb{E}[\right.}\left.\left.\pi^{\mathrm{mf}}(t, y(t)+C(t))-\pi^{\mathrm{mf}}(t, y(t))\right]\right] v_{y}\left(\pi^{\mathrm{mf}}\right)+\frac{1}{2}\left(b \pi_{y}^{\mathrm{mf}}\right)^{2} v_{y y}\left(\pi^{\mathrm{mf}}\right) \\
&+\lambda \mathbb{E}\left[v\left(\pi^{\mathrm{mf}}(t, y(t)+C(t))\right)-v\left(\pi^{\mathrm{mf}}(t, y(t))\right)\right]=0 .
\end{aligned}
$$

We can simplify this by dividing the whole equation by $v_{y}$ to obtain the pide for the forward term $\pi^{\mathrm{mf}}$ :

$$
\begin{aligned}
& \pi_{t}^{\mathrm{mf}}+a \pi_{y}^{\mathrm{mf}}+\frac{1}{2} b \pi_{y y}^{\mathrm{mf}}+\frac{1}{2} \frac{v_{y y}\left(\pi^{\mathrm{mf}}\right)}{v_{y}\left(\pi^{\mathrm{mf}}\right)}\left(b \pi_{y}^{\mathrm{mf}}\right)^{2} \\
& +\lambda \mathrm{E}\left[\pi^{\mathrm{mf}}(t, y(t)+C(t))-\pi^{\mathrm{mf}}(t, y(t))+\frac{v\left(\pi^{\mathrm{mf}}(t, y(t)+C(t))\right)-v\left(\pi^{\mathrm{mf}}(t, y(t))\right)}{v_{y}\left(\pi^{\mathrm{mf}}(t, y(t))\right)}\right]=0 .
\end{aligned}
$$

Again we substitute for $\pi^{\mathrm{mf}}=e^{-r t} \pi^{\mathrm{m}}$ in (3.1.12). After we simplify the notation, the corresponding PIDE for the discounted Mean Value principle with jump is then

$$
\begin{aligned}
\pi_{t}^{\mathrm{m}} & +a \pi_{y}^{\mathrm{m}}+\frac{1}{2} b \pi_{y y}^{\mathrm{m}}+\frac{1}{2} \frac{v_{y y}\left(\pi^{\mathrm{m}}\right)}{v_{y}\left(\pi^{\mathrm{m}}\right)}\left(b \pi_{y}^{\mathrm{m}}\right)^{2}-r \pi^{\mathrm{m}} \\
& +\lambda \int\left(\pi^{\mathrm{m}}(t, y(t)+c)-\pi^{\mathrm{m}}(t, y(t))+\frac{v\left(\pi^{\mathrm{m}}(t, y(t)+c)\right)-v\left(\pi^{\mathrm{m}}(t, y(t))\right)}{v_{y}\left(\pi^{\mathrm{m}}(t, y(t))\right)}\right) v(d c)=0 .
\end{aligned}
$$

We recognize that the continuous part of the PIDE is the same as the related PDE for the Mean Variance principle in the diffusion case including a positive "local risk aversion" for increasing and convex function $V($ ). Conditional on the event of the jump with instantaneous rate of $\lambda$, the PIDE captures the effect of the premium jump by means of the term $\pi^{\mathrm{m}}(t, y(t)+C(t))-\pi^{\mathrm{m}}(t, y(t))$ as well as the relative difference of the convex function $v(\pi)$ as a result of the jump with respect to the differentiation of $v()$ without a jump. If we assume $v()$ as a nonlinear function, then the PIDE reflects the jump effect on the price in both linear and nonlinear sense.

\section{Standard-Deviation Pricing with Jump}

To obtain the time-consistent Standard-Deviation price we have to revalue the principle formula in (2.3.2) under the jump process:

$$
\pi^{\mathrm{s}}(t, y(t))=e^{-r \Delta t}\left(\mathbb{E}_{t}\left[\pi^{\mathrm{s}}(t+\Delta t, y(t+\Delta t))\right]+\beta \sqrt{\Delta t} \sqrt{\operatorname{Var}_{t}\left[\pi^{\mathrm{s}}(t+\Delta t, y(t+\Delta t))\right]}\right) .
$$


From equation (2.3.3) the equivalent continuous-time limit of the above price in terms of the infinitesimal generator is

$$
r \pi^{\mathrm{s}}=A_{J} \pi^{\mathrm{s}}+\beta \sqrt{A_{J} \pi^{\mathrm{s} 2}-2 \pi^{\mathrm{s}} \times A_{J} \pi^{\mathrm{s}}}
$$

where $\pi^{\mathrm{s}}$ and $A_{J} \pi^{\mathrm{s}}$ are functions of $(t, y(t))$. We can insert for $A_{J} \pi^{\mathrm{s}}$ from equation (3.1.2) and for $A_{J} \pi^{\mathrm{v} 2}-2 \pi \times A_{J} \pi^{\mathrm{v}}$ from equation (3.1.5) and hence we obtain the appropriate PIDE for the Standard-Deviation principle as below:

$$
\begin{aligned}
\left.\pi_{t}^{\mathrm{s}}+a \pi_{y}^{\mathrm{s}}+\frac{1}{2} b^{2} \pi_{y y}^{\mathrm{s}}-r \pi^{\mathrm{s}}+\lambda(t, y(t)) \int\left(\pi^{\mathrm{s}}(t, y(t)+c)\right)-\pi^{\mathrm{s}}(t, y(t))\right) v(d c) \\
+\beta \sqrt{\lambda(t, y(t)) \int\left(\pi^{\mathrm{s}}(t, y(t)+c)-\pi^{\mathrm{s}}(t, y(t))\right)^{2} v(d c)+\left(b \pi_{y}^{\mathrm{s}}\right)^{2}}=0 .
\end{aligned}
$$

The Standard-Deviation PIDE presents the jump effect on the premium by using the first and second moments of the premium jump $\pi^{\mathrm{s}} \pi^{\mathrm{s}}(y+c)-\pi^{\mathrm{s}}(y)$. The loading part of the equation with coefficient $\beta$ consists of the conditional quadratic premium jump and quadratic term $\left(b \pi_{y}^{\mathrm{s}}\right)^{2}$, where the square root function makes it impossible to rewrite a linear version of this PIDE. If there is no jump, $\lambda=0$, the PIDE will be summarized to the PDE in (2.3.4) or (2.3.5).

\section{Cost-of-Capital Principle with Jump}

The Cost-of-Capital premium principle can also be valued by assuming a jump process for the underlying insurance process. The one-step pricing formula is the same as equation (2.3.9). We start by recalling its equivalent version in (2.3.10) and we adapt the infinitesimal generator to the jump version.

$r \pi^{\mathrm{c}}(t, y(t))=A_{J} \pi^{\mathrm{c}}\left(t, y(t)+\delta \sqrt{\Delta t} \operatorname{VaR}_{q, t}\left[\lim _{\Delta t \rightarrow 0} \frac{\pi^{\mathrm{c}}(t+\Delta t, y(t+\Delta t))-\mathbb{E}_{t}\left[\pi^{\mathrm{c}}(t+\Delta t, y(t+\Delta t))\right]}{\Delta t}\right]\right.$.

Note that we multiplied $\mathrm{VaR}_{q, t}$ by $\sqrt{\Delta t}$ to scale down the annual $\mathrm{VaR}_{q}$ to the $\Delta t$-related version, $\operatorname{VaR}_{q_{\Delta t}}$. This is consistent with the usual Variance-Covariance method of calculating VaR. Using the Itô-Doeblin representations of $\pi^{\mathrm{c}}(t+\Delta t, y(t+\Delta t))$ the limit under 
VaR can be rearranged as

$$
\begin{aligned}
& \lim _{\Delta t \rightarrow 0} \frac{\pi^{\mathrm{c}}(t, y(t))-\mathbb{E}_{t}\left[\pi^{\mathrm{c}}(t+\Delta t, y(t+\Delta t))\right]}{\Delta t} \\
& \quad+\lim _{\Delta t \rightarrow 0} \frac{1}{\Delta t} \int_{t}^{t+\Delta t}\left(\pi_{t}^{\mathrm{c}}(s, y(s))+a \pi_{y}^{\mathrm{c}}(s, y(s))+\frac{1}{2} b^{2} \pi_{y y}^{\mathrm{c}}(s, y(s))\right) d s \\
& +\lim _{\Delta t \rightarrow 0} \frac{1}{\Delta t}\left[\int_{t}^{t+\Delta t} b \pi_{y}^{\mathrm{c}}(s, y(s)) d W(s)+\sum_{t<s \leq t+\Delta t}\left[\pi^{\mathrm{c}}(s, y(s)+C(s))-\pi^{\mathrm{c}}(s, y(s))\right] .\right.
\end{aligned}
$$

The first term, by definition of the infinitesimal generator, is equal to $-A_{J} \pi^{\mathrm{v}}(t, y(t))$. The second limit by equation (2.3.12) will be equal to

$$
\pi_{t}^{\mathrm{c}}(t, y(t))+a \pi_{y}^{\mathrm{c}}(t, y(t))+\frac{1}{2} b^{2} \pi_{y y}^{\mathrm{c}}(t, y(t)) .
$$

We refer to the last term later. By using equation (3.1.2) to substitute for $A_{J} \pi^{\mathrm{v}}(t, y(t))$, the summation of the first two terms in (3.1.17) will be equal to $-\lambda(t, y(t)) \mathbb{E}\left[\pi^{\mathrm{c}}(t, y(t)+C(t))-\pi^{\mathrm{c}}(t, y(t))\right]$. By translation invariance for the VaR operator, the expectation term can be factorized and then its limit will be zero as

$$
\lim _{\Delta t \rightarrow 0} \delta \sqrt{\Delta t}\left[\lambda(t, y(t)) \mathbb{E}\left[\pi^{\mathrm{c}}(t, y(t)+C(t))-\pi^{\mathrm{c}}(t, y(t))\right]\right]=0 .
$$

Hence, equation (3.1.16) will be rearranged as

$$
\begin{aligned}
& r \pi^{\mathrm{c}}(t, y(t))=\pi_{t}^{\mathrm{c}}(t, y(t))+a \pi_{y}^{\mathrm{c}}(t, y(t))+\frac{1}{2} b^{2} \pi_{y y}^{\mathrm{c}}(t, y(t))+\lambda \mathbb{E}\left[\pi^{\mathrm{c}}(t, y(t)+C(t))-\pi^{\mathrm{c}}(t, y(t))\right] \\
& +\lim _{\Delta t \rightarrow 0} \frac{\delta}{\sqrt{\Delta t}} \operatorname{VaR}_{q, t}\left[\int_{t}^{t+\Delta t} b \pi_{y}^{\mathrm{c}}(s, y(s)) d W(s)+\sum_{t<s \leq t+\Delta t}\left[\pi^{\mathrm{c}}(s, y(s)+C(s))-\pi^{\mathrm{c}}(s, y(s))\right]\right],
\end{aligned}
$$

where we substitute for $A_{J} \pi^{\mathrm{v}}(t, y(t))$ from (3.1.2).

To compute this premium, we need some insights into the distribution of the process under the VaR term. The whole terms under the VaR function are a special Lévy jump-diffusion process containing: a Brownian motion with drift zero and diffusion $b(s, y(s)) \pi_{y}^{\mathrm{c}}(s, y(s))$ and a compound Poisson process for a jump component with intensity $\lambda \Delta t$, compensated by its expected value between $(t, t+\Delta t)$. If we assume stationary and independent increments, it is possible to identify the characteristic function of the above Lévy process and find its marginal distribution.

In equation (2.3.17) in Subection 2.3 we inferred that the limit of the Itô integral in the VaR operator in (3.1.19) is normally distributed with variance $\Delta t\left(b \pi_{y}\right)^{2}$. The summation term $X=\sum_{t<s \leq t+\Delta t}\left[\pi^{\mathrm{c}}(s, y(s)+C(s))-\pi^{\mathrm{c}}(s, y(s))\right]$, however, is a compound Poisson process with intensity $\lambda \Delta t$. Therefore, the terms under the VaR operator in (3.1.19) con- 
stitute a convolution. We assume that the Itô integral and compound Poisson jumps are independent, as so are the frequency and size of the premium jump, and we calculate the characteristic function $\psi(\theta)$ of the convolution. We denote the convolution of the normal and compound Poisson random variables by $M=Z(t+\Delta t)+X$. Note that, under the VaR operator in equation (3.1.19), $M$ is divided by $\sqrt{\Delta t}$. Hence, considering the fact that $\psi_{\frac{M}{\sqrt{\Delta t}}}(\theta)=\psi_{M}\left(\frac{\theta}{\sqrt{\Delta t}}\right)$ and the independence assumption, the characteristic function of the convolution under VaR is

$$
\begin{aligned}
\psi_{\frac{M}{\sqrt{\Delta t}}}(\theta) & =\exp \left[-\frac{\Delta t\left(b \pi_{y}\right)^{2}\left(\frac{\theta}{\sqrt{\Delta t}}\right)^{2}}{2}+\lambda \Delta t\left(\psi_{X}\left(\frac{\theta}{\sqrt{\Delta t}}\right)-1\right)\right] \\
& =\exp \left[-\frac{\left(b \pi_{y} \theta\right)^{2}}{2}+\lambda \Delta t\left(\psi_{X}\left(\frac{\theta}{\sqrt{\Delta t}}\right)-1\right)\right] .
\end{aligned}
$$

The distribution of the convolution depends on the distribution of the premium jump and thus on the form of $\psi_{X}$. If we assume normally distributed premium jumps, $D \sim$ $N\left(\mu, \sigma^{2}\right)$, the characteristic function of the whole convolution turns to

$$
\begin{aligned}
\psi_{\frac{M}{\sqrt{\Delta t}}}(\theta) & =\exp \left[-\frac{\left(b \pi_{y} \theta\right)^{2}}{2}+\lambda \Delta t\left(\frac{i \mu \theta}{\sqrt{\Delta t}}-\frac{\sigma^{2} \theta^{2}}{2 \Delta t}-1\right)\right] \\
& =\exp \left[-\frac{\left(b \pi_{y} \theta\right)^{2}}{2}+\lambda \sqrt{\Delta t}(i \mu \theta)-\frac{\lambda \sigma^{2} \theta^{2}}{2}-\lambda \Delta t\right] .
\end{aligned}
$$

If we take the limit of $\psi_{\frac{M}{\sqrt{\Delta t}}}(\theta)$ when $\Delta t \rightarrow 0$, we obtain

$$
\lim _{\Delta t \rightarrow 0} \psi_{\frac{M}{\sqrt{\Delta t}}}(\theta)=\exp \left[-\frac{b^{2} \pi_{y}^{2} \theta^{2}}{2}-\frac{\lambda \sigma^{2} \theta^{2}}{2}\right]
$$

which shows that the asymptotic distribution of the compound Poisson process with coefficient $1 / \sqrt{\Delta t}$ is normal with mean zero and variance $\lambda \sigma^{2}$, where the zero mean was justified earlier in (3.1.18). Hence, the convolution is normal with mean zero and variance $b^{2} \pi_{y}^{2}+\lambda \sigma^{2}$, and by using the scale invariance property, the limit of the VaR term in (3.1.19) will be equal to $\sqrt{b^{2}(t, y(t)) \pi^{\mathrm{c} 2}{ }_{y}(t, y(t))+\lambda(t, y(t)) \sigma^{2}} \times \Phi^{-1}(q)$. Finally (3.1.19) gives the resulted PIDE as

$$
\begin{aligned}
r \pi^{\mathrm{c}}(t, y(t))= & \pi_{t}^{\mathrm{c}}(t, y(t))+a(t, y(t)) \pi_{y}^{\mathrm{c}}(t, y(t))+\frac{1}{2} b^{2}(t, y(t)) \pi_{y y}^{\mathrm{c}}(t, y(t)) \\
& +\delta \Phi^{-1}(q) \sqrt{b^{2}(t, y(t)) \pi^{\mathrm{c} 2}(t, y(t))+\lambda \operatorname{Var}\left[\pi^{\mathrm{c}}(t, y(t)+C(t))-\pi^{\mathrm{c}}(t, y(t))\right]} \\
& +\lambda \mathbb{E}\left[\pi^{\mathrm{c}}(t, y(t)+C(t))-\pi^{\mathrm{c}}(t, y(t))\right],
\end{aligned}
$$


where taking $\Phi^{-1}(q)=k$ and changing to integral notation, the PIDE is:

$$
\begin{aligned}
\pi_{t}^{\mathrm{c}}+a \pi_{y}^{\mathrm{c}}+\frac{1}{2} b^{2} \pi_{y y}^{\mathrm{c}}- & r \pi^{\mathrm{c}}+\lambda \int\left(\left(\pi^{\mathrm{s}}(y(t)+c)-\pi^{\mathrm{s}}(y(t))\right)\right) v(d c) \\
& +\delta k \sqrt{b^{2} \pi^{\mathrm{c} 2} y+\lambda \int \operatorname{Var}\left[\pi^{\mathrm{c}}(t, y(t)+C(t))-\pi^{\mathrm{c}}(t, y(t))\right] v(d c)}=0 .
\end{aligned}
$$

Looking back at the derivation of the PIDE, it is clear that the loading term of the premium (VaR term) is independent of the expected premium jump. The PIDE also shows that if the premium jump is normally distributed, the Cost-of-Capital price is able to capture a quadratic jump effect on the price (i.e. the variance of the premium jump size) that makes it very similar to the Standard-Deviation price, which presents the second moment of the premium jump. The rest of the terms for the Cost-of-Capital and StandardDeviation prices are the same. The quadratic driver of the PIDE is forced to be linearized by the square root function in both of the Standard-Deviation and Cost-of-Capital principles. In the non-jump case, the PIDE converges the PDE in (2.3.19).

The underlying distribution of the premium jump size is effective on the Cost-ofCapital price of the insurance process with jump. If we change the distribution of the premium jump, the continuous-time limit of the Cost-of-Capital premium will result in a different PIDE. For example, if the premium jump has an exponential distribution with parameter $\alpha$, then it will turn (3.1.20) into

$$
\begin{aligned}
\psi_{\frac{M}{\sqrt{\Delta t}}}(\theta) & =\exp \left[-\frac{\left(b \pi_{y} \theta\right)^{2}}{2}+\lambda \Delta t\left(\left(1-i \frac{\theta}{\sqrt{\Delta t}} \alpha^{-1}\right)^{-1}-1\right)\right] \\
& =\exp \left[-\frac{\left(b \pi_{y} \theta\right)^{2}}{2}+\lambda \Delta t\left(\frac{i \theta}{\lambda \sqrt{\Delta t}-i \theta}\right)\right]
\end{aligned}
$$

and by taking the limit when $\Delta t \rightarrow 0$, the exponential part tends to zero and we have

$$
\lim _{\Delta t \rightarrow 0} \psi_{\frac{M}{\sqrt{\Delta t}}}(\theta)=\exp \left[-\frac{b^{2} \pi_{y}^{2} \theta^{2}}{2}\right] .
$$

This is the characteristic function of the normal distribution with mean zero and variance $b^{2} \pi_{y}^{\mathrm{c} 2}$ and by means of equation (3.1.19) gives the PIDE as

$$
\pi_{t}^{\mathrm{c}}+a \pi_{y}^{\mathrm{c}}+\frac{1}{2} b^{2} \pi_{y y}^{\mathrm{c}}+\delta k\left|b \pi_{y}^{\mathrm{c}}\right|-r \pi^{\mathrm{c}}+\lambda \int\left(\left(\pi^{\mathrm{s}}(y(t)+c)-\pi^{\mathrm{s}}(y(t))\right)\right) v(d c)=0 .
$$

We observe a different PIDE in the sense that the quadratic jump term has disappeared from the VaR perspective and only the jump effect is captured via the expectation term of the Cost-of-Capital premium principle. The non-jump case still converges to the Cost-of- 
Capital PDE in (2.3.19).

\subsection{Numerical Example}

In this section we apply the idea of time-consistent valuation to price a simplified insurance contract to give a real-world example of this method and its differences to the normal one-step valuation. We apply the multi-step pricing operator to the time-consistent version and divide any time period $T-t$ into $n$ steps with a length of $\Delta t$. We use the same backward iteration method to calculate the value of the premium for an insurance risk. As we modeled earlier, the unhedgeable risk process can be described either by a simple diffusion process in (4.2.1) or a jump-diffusion process in (3.1.1). In time step $(t, t+\Delta t)$, we have the increment as below,

$$
\begin{array}{ll}
\text { Simple Diffusion: } & \Delta y(t)=\mu(t, y(t)) \Delta t+\sigma(t, y(t)) \Delta W(t) \\
\text { jump-diffusion: } & \Delta y(t)=\mu(t, y(t)) \Delta t+\sigma(t, y(t)) \Delta W(t)+C(t, y(t)) \Delta N(t) .
\end{array}
$$

We are interested in the price of any contract at time $t \geq 0$ that offers a contingent payoff at $T$ or any time depending on $T$. To price the contract, we will use the premium principles that we used in the time-consistent contexts in the previous sections. To implement the idea of time-consistent valuation, we will use the Markov chain method to approximate the underlying process and payoff function, where the pricing rules will be one of the previously mentioned premium principles. The Markov chain provides a straightforward method to apply the valuation task in each sub-period for the payoff and calculate the price in a dynamic way. This method is frequently used to price path dependent derivatives such as American options, barrier options, etc. See for example Duan et al. (2003) and Monoyios (2004).

\section{Setting for a Simple Life Insurance Payoff}

Suppose we have a stylized life-insurance contract for the period of $[0, T]$. We are monitoring the health of an individual as a diffusion process, say $y(t)$. The person is alive as long as $y(t)>0$ and dies when $y(t)$ hits zero. Therefore, the insurance contract has a payoff 1 at time $T$ (i.e. the survival benefit), if $y(t)>0$ for all $0<t<T$. Another stylized contract pays the benefit 1 at $T$ if $y(t)$ hits the level zero before $T$, where the individual dies. Let us define the first hitting time at level $x>0$ for the process $y(t)$ as below,

$$
\tau_{x}=\min \{t \geq 0 ; \quad y(t)=x\}
$$

If we assume $y(t)=W(t)$ is a Brownian motion, it is not hard to prove that $\mathbb{P}\left(\tau_{x}<\infty\right)=$ 1 but $\mathbb{E}\left(\tau_{x}\right)=\infty$. The health process can offer a more realistic picture if we assume a 
negative drift $\mu<0$ as any individual's health gradually deteriorates and the individual comes closer to death. Naturally, the health quality of an individual can fluctuate daily due to different factors like nutrition, exercise, diseases etc, which means $\sigma>0$.

Based on the above properties of the Brownian motion $W(t)$, such as "symmetry", for a constant $\mu$ and $\sigma$, the distribution function of the first hitting time of the level zero by the process $y$ with the initial value of $y(t)$ and the maturity time $T$ is,

$$
\mathbb{P}\left(\tau_{0}<T-t \mid y(t)\right)=\Phi\left(\frac{-y(t)-\mu(T-t)}{\sigma \sqrt{T-t}}\right)+\exp \left(\frac{-2 \mu y(t)}{\sigma^{2}}\right) \Phi\left(\frac{-y(t)+\mu(T-t)}{\sigma \sqrt{T-t}}\right) .
$$

We will use this probability and the corresponding survival function for the hitting time $\tau_{0}$ to calculate the analytical solution of the PDEs obtained for each premium principle.

The physical setting for the value and payoff of the above stylized product is basically a simple control problem for the underlying stochastic process with constant boundary levels over time. It is ideal and more realistic, regarding the natural situation of any individual, that $\mu(t, y(t))$ and $\sigma(t, y(t))$ be stochastic processes depending on time and the health condition of the individual in the previous time step. However, to keep our demonstration simple, we assume a constant $\mu$ and $\sigma$ in this paper.

\section{Markov Chain Implementation}

The Markov chain method has been used extensively as a numerical tool for control problems, particularly in the dynamic valuation of contingent payoffs such as American options. See for example Kushner and Dupuis (2001) and Yin and Zhang (2012). The backward iteration of the one step valuation can be applied by means of the Markov chain method to the underlying (original) health process, discretized by both time horizon and state space. We define the approximating Markov chain on the related state space by using a finite difference interval $\Delta y$ such that the first moments of the chain are matched to those of the original process $y(t)$, as $\Delta y \rightarrow 0$. Note that $\Delta y$ can also be interpreted as a discrete time parameter of the Markov chain and can be defined as a function of time step $\Delta t$.

\section{Pricing by Simple Diffusion Health Process}

We start with a term life insurance for time horizon $T$ that pays benefit 1 at time $T$ on the event of death if $\tau_{0} \in(0, T)$ and pays zero otherwise. This is in fact a path-dependent derivative similar to a European style "down-and-in" barrier option with barrier level zero. If the process hits zero before $T$, the beneficiaries make sure they will receive a payoff with present value $1 \times e^{-r\left(T-\tau_{0}\right)}$ at $\tau_{0}$.

We use the Variance premium principle as the pricing rule. In a continuous-time setting, recalling the equation (2.2.16), the time-consistent valuation of the above contract 
will result in the following pde,

$$
\pi_{t}^{\mathrm{v}}+\mu \pi_{y}^{\mathrm{v}}+\frac{1}{2} \sigma^{2} \pi_{y y}^{\mathrm{v}}+\frac{1}{2} \alpha\left(\sigma \pi_{y}^{\mathrm{v}}\right)^{2}-r \pi^{\mathrm{v}}=0 .
$$

with the domain $\{(t, y(t)) ; 0 \leq t \leq T, 0 \leq y(t)<\infty\}$ and the boundary conditions

$$
\begin{array}{lrlrl}
\pi^{\mathrm{V}}(t, 0) & =1 \times e^{-r(T-t)} & & 0<t<T \\
\pi^{\mathrm{V}}(T, y) & =0 & , & y>0
\end{array}
$$

and the terminal condition $\pi^{\mathrm{v}}(T, y(T))=\mathbb{1}_{\left\{0 \leq \tau_{0}<T\right\}}$. We implicitly assume that, if for any $t \leq T, y(t)$ hits zero, the process will be killed and will remain zero till time $T$ when the payoff will be made.

Basically, we use a Markov chain with a lattice structure of approximation for $y(t)$ in a discrete-time and finite state space. Duan et al. (2003) have provided a generally applied frame for the method used to price American option, by applying the Black-Scholes model and GARCH option pricing model. The time space consists of the number of time steps $\Delta t$, and the payoff can be recursively defined as below for all $s \in\{t, t+\Delta t, t+2 \Delta t, \ldots, T-\Delta t\}$ :

$$
\pi^{\mathrm{V}}(s, y(s))=\mathbb{E}\left[e^{-r \Delta t} \pi^{\mathrm{v}}(s+\Delta t, y(s+\Delta t)) \mid \mathscr{F}_{s}\right]+\frac{1}{2} \alpha \operatorname{Var}\left[e^{-r \Delta t} \pi^{\mathrm{v}}(s+\Delta t, y(s+\Delta t)) \mid \mathscr{F}_{s}\right] .
$$

We repeat this valuation operation in the backward iteration method to price the product at time zero, starting from $B(T, y(T))$. As we mentioned before, we use constant interest rate, drift rate and volatility.

To implement the Markov chain, we select a upper boundary $y_{\max }$ as

$$
=y(0)+k \sigma \sqrt{T}
$$

where $\sigma \sqrt{T}$ is the standard deviation of $y(t)$ over $[0, T]$. This will reduce the domain into $[0, T] \times\left[0, y_{\max }\right]$ and add extra boundary condition $\pi^{\mathrm{v}}\left(t, y_{\max }\right)=0,0<t<T$ to the ones in equation (3.2.3) where $y_{\max }$ acts like a European style "up-and-out" barrier option. Although the probability of hitting $y_{\max }$ will be negligible for a reasonably large $k$ and negative drift, we will later modify the sample space in the calculation phase by conditioning the probability on the over- $y_{\max }$ hits.

For a $y(t)$ modeled by simple diffusion, the transition matrix can be obtained via the method in Duan et al. (2003), which calculates the transition probabilities over all states in the range $\left(0, y_{\max }\right)$. We use the "adaptive recombining trinomial tree" technique, in which the middle tree node follows the local drift and the up/down nodes follow the volatility for each time step. See for example, Tang and $\mathrm{Li}$ (2007) for more details about the method. We match the local mean and variance of the underlying process and the Markov state space. 
The state difference interval will be constructed as

$$
\Delta y(t)=\left\{\begin{aligned}
\Delta y_{d}(t) & =-\sigma \sqrt{k \Delta t} \\
\Delta y_{m}(t) & =0, \\
\Delta y_{u}(t) & =\sigma \sqrt{k \Delta t}
\end{aligned}\right.
$$

where a common value of $k=3$ also can match the local kurtosis and reduce the distribution error to speed up the convergence of the chain. Similar method in Figlewski and Gao (1999) and Baule and Wilkens (2004), produced the trinomial transition probabilities as follows

$$
\begin{aligned}
p_{d} & =1 / 6-\frac{\mu \sqrt{3 \Delta t}}{6 \sigma} \\
p_{m} & =2 / 3, \\
p_{u} & =1 / 6+\frac{\mu \sqrt{3 \Delta t}}{6 \sigma}
\end{aligned}
$$

where $p_{u} \geq 0, p_{m} \geq 0, p_{d} \geq 0$ and $p_{u}+p_{m}+p_{d}=1$ and the state difference interval is constructed so that the local kurtosis will be matched and the distribution error will decline. For any transition that leads to a state reaching the boundary levels $y=0$ and $y=y_{\text {max }}$, the process will be killed by setting the corresponding transition probability equal to 1 . The same is valid, for the jump-diffusion case in the next subsection. The result for the scope of our stylized example is consistent with the nature of the health process, where for a negative drift $\mu$ we expect a larger downward probability $p_{d}$ (and smaller upward probability $p_{u}$ ), to push the process closer to zero.

\section{Pricing by jump-diffusion Health Process}

We enter a simple jump component into the trinomial tree to investigate its effect on the price of the product. Generally, most of the methods for random-sized Poisson jump components are studied with the aim of finding the tree probabilities so that the discrete time Markov process including a jump matches the first local moments of the continuous-time jump-diffusion process. For more about the applications of the method to price the options, see for example Amin (1993) and Yuen and Yang (2009).

Considering the same criteria, Hilliard and Schwartz (2005) investigated how to use a jump-diffusion model to price derivatives. They used a bivariate tree approach to separate the diffusion and jump parts and used the same methods to match the local moments. They assumed that the size of the jump in discrete time also has a grid containing jump nodes constructed by the integer product of the jump size's finite difference interval. After that, the jump-diffusion discrete time approximation will be the summation of the diffusion and jump parts.

We use a simplified version of the above techniques to separate the jump and diffusion parts in the implemented Markov chain setting. To keep the problem simple, we assume 
a constant jump size $J$ such that

$$
\left\lceil\frac{J}{\Delta y(s)}\right\rceil=K
$$

where $K \geq 2$. As the number of valuation steps increases, the state difference $\Delta y(s)$ decreases and $K$ increases so that $J$ remains constant.

We also implement the transition probabilities for a valuation time step $\Delta t$, in the form of a skewed quadrinomial, by mixing the arrival time rate of jump $\lambda$ and trinomial tree transition probabilities as below,

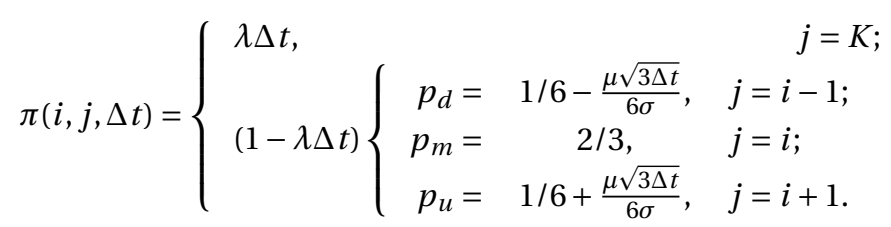

Based on this formulation, we assume that any jump event, will be large enough to nullify the effect of the diffusion part for the evolution of the underlying health process. If there is no jump, we can reduce the sample space for the diffusion part and distort the trinomial transition probabilities so that we can define the entire process in one probability space. This can be considered as a very simple and special case of the regime switching between the jump and diffusion parts, so that there is only a possible jump in the first regime and diffusion instead of a jump in the second regime.

\section{Simulation}

We apply the above method to calculate the time-consistent price of the contract with both diffusion and jump-diffusion processes. To compare the time-consistent price obtained from the diffusion and jump process, we also need to match the local moments of the diffusion process with regard to those of jump process. Therefore, we recall the locally matched processes for constant drift, volatility and jump size as below,

$$
\begin{array}{ll}
\text { Simple Diffusion: } & d y(t)=(\mu+\lambda J) d t+\left(\sqrt{\sigma^{2}+\lambda J}\right) d W(t) \\
\text { jump-diffusion: } & d y(t)=\mu(t, y(t)) d t+\sigma d W(t)+J d N(t) .
\end{array}
$$

In the above formulation we implicitly assume that no more than one jump should be possible for a small time step. Using the locally matched diffusion process above and (3.2.8), 
we update the transition probabilities in (3.2.7) as

$$
\left\{\begin{array}{l}
p_{d}=1 / 6-\frac{(\mu+\lambda K \Delta y) \sqrt{3 \Delta t}}{6 \sqrt{\sigma^{2}+\lambda K \Delta y}} \\
p_{m}=2 / 3 \\
p_{u}=1 / 6+\frac{(\mu+\lambda K \Delta y) \sqrt{3 \Delta t}}{6 \sqrt{\sigma^{2}+\lambda K \Delta y}} .
\end{array}\right.
$$

The alternative transition probabilities for the jump case stays the same as (3.2.9).

\section{Variance Price}

We calculate the time-consistent Variance premium principle for a $T$-year term life insurance. We do this for both the death and survival benefits based on the stylized health process. Note that in this numerical work, we do not solve the related Variance PDE, but we directly calculate the Variance premium for the shorter time steps starting with the terminal time $T$ state space and apply the backward iteration method to reach the time $t<T$ price.

It is important to examine the convergence of the Markov chain trinomial tree approximation to the analytical time-consistent price. The time-consistent solution for the case of the Variance price was derived in (2.2.17) as $\pi^{\mathrm{v}}(t, y)=$ $\frac{X_{0} e^{r t}}{\gamma} \ln \mathbb{E}\left[\exp \left(\frac{\gamma}{X_{0} e^{r T}} f(y(T))\right) \mid y(t)=y\right]$. According to the Markov chain discretization, the payoff for the death benefit is 1 when $\tau_{0}<T-t$ and 0 in all other cases. The apposite is valid for the survival benefit where the payoff is 1 if $\tau_{0} \geq T-t$. If we assume $\mathbb{P}\left(\tau_{0}<T\right)=p$ as the probability of a Bernoulli event, which can be calculated by the equation (3.2.1), the analytical price will be obtained as

$$
\pi^{\mathrm{v}}(t, y)=\frac{X_{0} e^{r t}}{\gamma} \ln \mathbb{E}\left[e^{\frac{\gamma}{X_{0} e^{r T}} \mathbb{I}_{\left\{\tau_{0}<T-t\right\}}} \mid y(t)=y\right]=\frac{X_{0} e^{r t}}{\gamma} \ln \left(1-p+p e^{\frac{\gamma}{X_{0} e^{r T}}}\right),
$$

where for $\alpha=\gamma / X_{0} e^{r t}$, the simpler notation is $\pi^{\mathrm{v}}(t, y)=\frac{1}{\alpha} \ln \left[1-p+p \times \exp \left(\alpha e^{r(T-t)}\right)\right]$.

We calculate the time-consistent price approximation for both types of coverage, based on the following set of sample parameters: the drift $\mu=-0.2$, the diffusion coefficient $\sigma=0.4$, the initial value at time $t, y(t)=1$, the time duration $T-t=1$, annual discount rate $r=0.05$, the relative risk aversion per benchmark wealth level $\frac{\gamma}{X_{0}}=0.1$, and the jump arrival time rate $\lambda=0.03$, the expected jump size $K=0.7 y(t)=0.7$, and the upper bound of the $y(T)$ state space will be driven as $\left.y_{\max }=y(t)+3 \sigma \sqrt{(} T-t\right)=2.2$. The probability of the first hitting time of the level zero (lower bound of the state space of $y(T)$ ), or equivalently the individual's death probability, can be calculated by (3.2.1) as $P\left(\tau_{y(\tau)=0} \leq T-t \mid y(t)=1\right)=p=0.03375$. Similarly the alternative conditional hitting time probability when taking into account the sample space reduction by the upper bound 
$y_{\max }=2.2$ for $k=3$ will be

$$
P\left(\tau_{y(\tau)=0}<T-t \mid y(t)=1, y(\tau) \leq y_{\max }\right)=\frac{p}{P\left(y(\tau) \leq y_{\max }\right)}=\frac{0.03375}{0.99967}=0.033758,
$$

where clearly the survival probability is $q=1-p=0.96624$. Using the equation (3.2.12), the analytical time-consistent Variance price for the life insurance coverage will be $\pi_{\text {Death }}^{\mathrm{v}}(t, y)=0.03363$ and $\pi_{\text {Survival }}^{\mathrm{v}}(t, y)=0.92055$.

We provide a numerical approximation of the time-consistent Variance price operator for both death and survival benefits using simple diffusion and jump settings in discrete time. We use the transition probabilities in equations (3.2.7) and (3.2.9) and kill the process for the transitions leading to the boundary conditions in equations (3.2.3). We then implement the backward iteration method, whereby the time steps $\Delta t$ become smaller when increasing the number of iterations, and we examine whether our approximation converges to the analytical continuous-time limit of the price.

Figure 3.1 represents the convergence of the Markov chain trinomial tree approximation to the analytical time-consistent Variance premium for the diffusion case in which the number of time steps $(n)$ increases and the parameters are the same as above. Although we have no analytical solution for the obtained PIDE in the jump case in 3.1.6, the Variance price converges to the certain levels of 0.0499 and 0.9057 for the death and survival coverage, respectively. The difference in the price is reasonable as we have a one-sided downward jump in the health process.

We still observe some perturbation in the Markov chain approximation, but the level of the relative difference between the values (i.e. the typical error) decreases when the number of steps increase. Figlewski and Gao (1999) explain that the reason for the typical errors is the lack of coincidence between the theoretical boundary levels and the highest state in the Markov chain. In our case, there is a lack of coincidence for the position of the time $t$ Markov chain premium in the lattice model with the analytical price, which always cause over/under value. Applying this method to the Standard-Deviation principle will give the same convergence result for both the diffusion and jump cases.

\section{Cost-of-Capital Price}

We also compute the Markov chain approximation of the time-consistent Cost-of-Capital price for the above life insurance contract. The analytical solution of the Cost-of-Capital PDE for the diffusion case is given by the equation (2.3.6) under the risk-adjusted underlying process (2.3.20) as below,

$$
\pi^{\mathrm{V}}(t, y)=\mathbb{E}\left[e^{-r(T-t)} f(y(T)) \mid y(t)=y\right]=\mathbb{E}_{t}\left[e^{-r(T-t)} \mathbb{I}_{\left\{\tau_{0}<T-t\right\}}\right]=e^{-r(T-t)} \times p,
$$



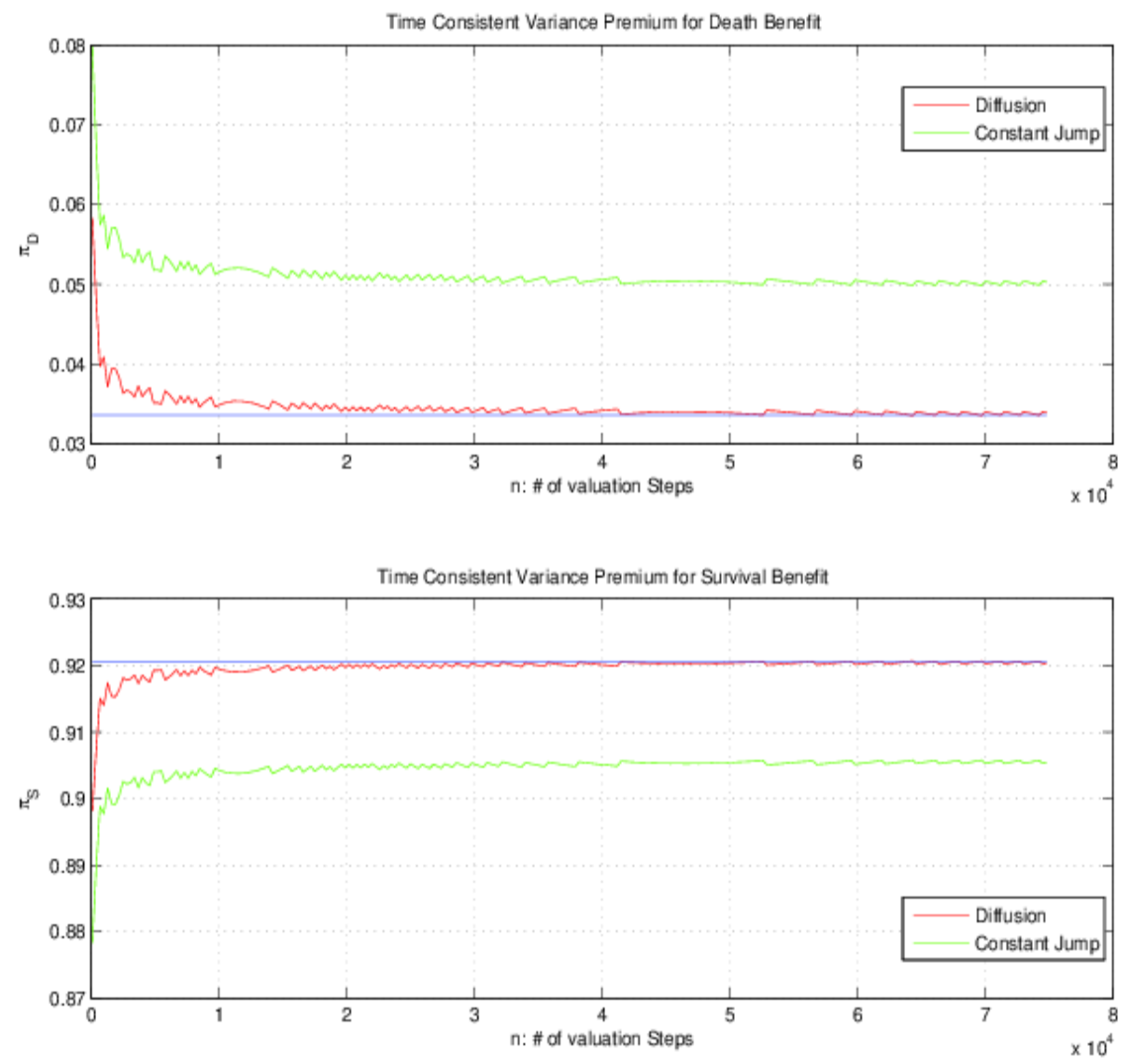

Figure 3.1: Markov Chain Simulation of the Time-Consistent Variance Premium for the Stylized Life Insurance Contract 
where $p=\mathbb{P}\left(\tau_{0}<T\right)$. There is no analytical solution for the jump-diffusion case. For the parameter values, we use the cost of capital $\delta=0.1$ instead of the relative risk aversion. In order to give a better picture of the approximation evolution, we choose a relatively high jump intensity $\lambda=0.1$ and probability level of the VaR, $1-q=0.999$. The rest of the parameters are the same as those that we used in Variance pricing.

We use (3.2.10) as the underlying process. Since the payoff for the death benefit decreases monotonically in $y$, we use $(a+\lambda J-\delta k b)$ as the downward adjustment for the drift rate. The adjustment calculates the upwind price of the insurance risk as the drift rate decreases more by $-\delta k b$, pushing the process more towards the zero level, which means a higher probability of death from the insurer's perspective. Using the equation (3.2.1), the probability of the first hitting time of the level zero (death probability) is computed as $p=0.04342$, where the conditional probability given the upper bound $y_{\text {max }}=2.2$, is $P\left(\tau_{0}<1 \mid y(t)=1, y(\tau) \leq 2.2\right)=0.043435$. On the other hand, since the survival benefit increases monotonically in $y$, we have to use $(a+\lambda J+\delta k b)$ as the upward adjustment for the drift rate, which gives a lower probability of hitting zero. This is interpreted as a higher price of the survival coverage for the insurer. Therefore, we obtain, $P\left(\tau_{0} \geq 1 \mid y(t)=1, y(\tau) \leq 2.2\right)=0.0 .98976$. By using the formulation in (3.2.13), we obtain the analytical time-consistent value of the Cost-of-Capital premium for the life insurance coverage as $\pi_{\text {Death }}^{\mathrm{c}}(t, y(t))=0.04132$ and $\pi_{\text {Survival }}^{\mathrm{c}}(t, y(t))=0.9416$.

Figure 3.2 illustrates the Markov chain approximation of the time-consistent value of the Cost-of-Capital premium for different number of valuation steps in the backward iteration method. The upper graph illustrates the premium of the death coverage under the diffusion and jump-diffusion process, while the lower graph shows the same premium for the survival coverage. We start the valuation with just $n=4$ steps and add four more steps to $n$ each time. In the above parameter set, the horizontal line is the analytical value of the time-consistent premium. In the case of death coverage modeled by a simple diffusion process, which increases the number of valuation steps, we observe a fast convergence of the Markov chain method to the analytical value.

However, for the jump-diffusion process, there is a downfall in the Markov chain approximation of the Cost-of-Capital premium on $n=100$. The reason for this dramatic reduction of the premium can be explained by the fact that, when the probability of the jump event at any time interval $(t, t+\Delta t)$ is less than the VaR probability threshold in that period, $\lambda \Delta t<q$, the $\mathrm{VaR}_{1-q}$ function is not able to capture the effect of the jump. Therefore, in the point where $\lambda \Delta t=q$ and after that, the premium jump cannot be reflected in VaR, and the Cost-of-Capital premium drops. This is a substantial weakness in the Costof-Capital premium principle when dealing with rare jump events and it fails to capture part of the premium jump in the final value. In our example, for $\lambda=0.1$ and $q=0.001$, this happens when $n \geq 100, \lambda \Delta t \leq 0.001$. After the drop point, the Markov chain approximation converges to a special level of the premium that is significantly higher than the 

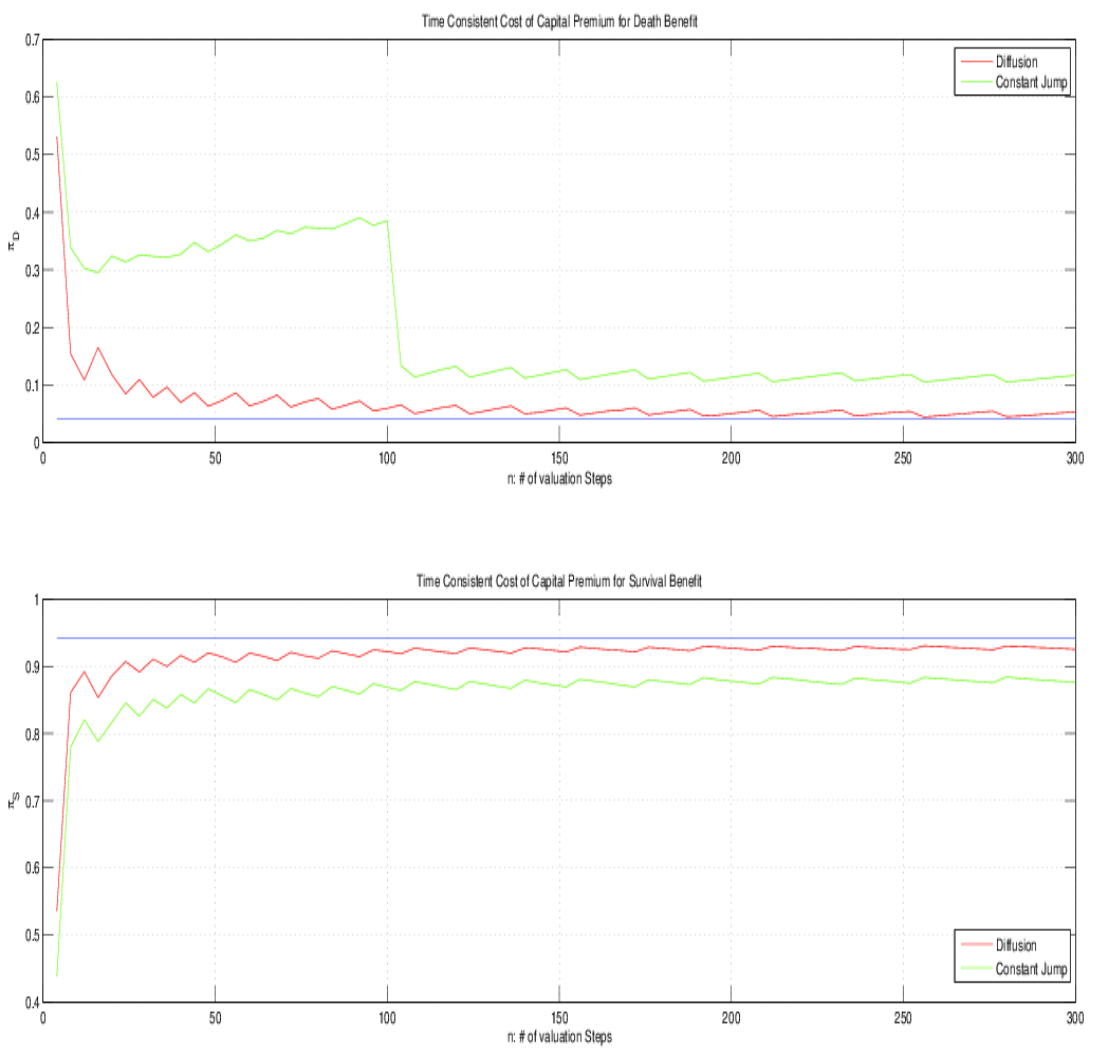

Figure 3.2: Markov Chain Simulation of the Time-Consistent Cost-of-Capital Premium for the Stylized Life Insurance Contract 
premium resulted by the simple diffusion process.

For the survival coverage, the Markov chain premium approximation obtained by the diffusion processes converges to the analytical value of the time-consistent Cost-ofCapital premium (horizontal line). In the jump case, we observe a normal convergence with a decreasing perturbation rate without any sudden increase or decrease in the premium, while the number of valuation steps increases. The reason for this is that we use a one-sided jump in our example that moves downwards and is located on the left hand side of the survival risk distribution. As a result, it is not able to stimulate the VaR function by means of the jump probability level $\lambda \Delta t$. Nevertheless, part of the jump effect is always captured by the expectation operator of the Cost-of-Capital principle and when comparing this to the diffusion case, this justifies the lower survival premium in the jump case in the second part of the Figure 3.2. 


\section{4}

\section{Market-Consistent Valuation under EIOPA Risk-margin and Time-consistency}

\section{This chapter is based on:}

Salahnejhad Ghalehjooghi, A. and Pelsser, A., (2016), Market-Consistent Actuarial Valuations with Applications to EIOPA Risk-margin and Time-consistent Pricing, under review in "Scandinavian Actuarial Journal".

Most life insurance and pension contracts are very long-dated liabilities that are not traded in the market. The supervisory requirement for a "Market-Consistent" valuation emphasizes the importance of pricing and risk management for such liabilities. To obtain a market-consistent price, we combine hedgeable financial risk with an (partially) unhedgeable actuarial risk and price the hybrid payoff using a "two-step market evaluation." In a general setting, the valuation process comprises the no-arbitrage price of pure financial risk, the value of partially hedged actuarial risk attributable to its correlation with financial risk (if available), and finally the value of pure actuarial risk through well-known actuarial premium principles. We implement a two-step valuation using a backward iteration method and obtain a time-consistent market-consistent (TCMC) price over the valuation period. We also provide a continuous-time limit of the TCMC price for the Variance and Standard-Deviation actuarial prices. We also provide a market-consistent version of the alternative pricing methods: the Best-Estimate pricing method typically used for pension liabilities and the EIOPA's Risk-margin method used under Solvency II to value life insurance liabilities. By comparing these prices with the TCMC price for a unit-linked contract, we show that the EIOPA Risk-margin method acts in the correct direction to reflect part of the uncertainty attributable to the future dynamics of unhedgeable risks, whereas BestEstimate pricing completely ignores that uncertainty. Because the Risk-margin method still ignores certain uncertainties, it is not fully time consistent and its gap with TCMC should not be ignored for long-dated contracts. 


\subsection{Market Consistent Valuation}

This section provides a general framework for a market-consistent valuation of insurance products or pension liabilities. We consider a class of contracts whose payoffs are contingent on the evolution of hedgeable financial risk(s) combined with unhedgeable (or partially hedgeable) actuarial risk(s). We first introduce the appropriate market-consistent valuation operator in a one-period setting. We then develop the framework for a multiperiod setting in which we construct the operator on the basis of the time-consistency property. For a contract with maturity $T$, the goal is to determine a market-consistent contract price at time $t \in[0, T)$.

\section{Setup and Assumption}

As a general setting, we start with $(\Omega, \mathscr{F}, \mathbb{P})$ as the underlying probability space with the filtered $\sigma$-algebra $\mathscr{F}_{t}$ on the finite interval $[0, T]$. Given the dual nature of the payoff, we form the required information flow using two separate $\sigma$-algebra consisting of $\left(\mathscr{F}_{t}^{S}\right)_{t \geq 0}$ for the financial information and $\left(\mathscr{G}_{t}^{A}\right)_{t \geq 0}$ for the actuarial information that the insurance company or the pension fund has available at time $t$. Essentially, our aim is to achieve an actuarial value conditional on the actuarial information represented by $\mathscr{G}^{A}$, which is generated through actuarial risk process(es) and not its financial value. We only use financial information to capture information on the traded financial risks in pursuit of market-consistency. Hence, we price the contract with respect to $\mathscr{G}^{A}$ and call the possible price/valuation operator " $\mathscr{G}^{A}$-conditional."

We denote the information flow of both risk categories at time $t$ by $\mathscr{G}_{t}=\sigma\left(\mathscr{G}_{t}^{A} \cup \mathscr{F}_{t}^{S}\right)$, where $\mathscr{G} \subset \mathscr{F}$. However later, given the required form of the valuation operator, we might need to use other versions of $\mathscr{G}$ where $\mathscr{G}^{A}$ and $\mathscr{F}^{S}$ have a different time-index representing the information flow in different points of time.

Suppose we have two stochastic processes $x_{t}$ as hedgeable pure financial risk and $y_{t}$ as non-traded unhedgeable insurance risk. The general payoff $G\left(x_{T}, y_{T}\right) \in L^{2}(\mathscr{F})$ is a mixed derivative of the financial and insurance risks in an incomplete market, whereas $G^{S}\left(x_{T}\right) \in$ $L^{2}(\mathscr{G})$ is a pure financial claim for which $L^{2}$ exhibits the space of the bounded random variables on the probability space. Let $\Pi_{\mathscr{G}}: L^{2}(\mathscr{F}) \rightarrow L^{2}(\mathscr{G})$ be the $\mathscr{G}$-conditional pricing operator.

We assume that the financial market is complete and arbitrage-free, which means that all risks conditional on actuarial information $\mathscr{G}^{A}$, only depends on financial risks and can be hedged (by trading in continuous-time). Hence, using the Fundamental Theorems of Asset Pricing (see, e.g., Delbaen and Schachermayer (1994)), there exists a unique martin- 
gale measure $\mathbb{Q}_{\mathscr{G}}$ for the financial risk process that is equivalent to $\mathbb{P}_{\mathscr{G}}$, such that

$$
\Pi_{\mathscr{G}}\left[G^{S}\right]=\mathbb{E}^{\mathrm{Q} \mathscr{G}}\left[G^{S}\right]
$$

Note that, the actuarial information may have some overlap with financial information such that it may make sense to use it even to price a pure financial payoff.

In contrast, we assume that actuarial risk is not (completely) hedgeable and is also rarely tradable. Thus, we cannot exploit a replicating portfolio argument for insurance derivatives. This turns to the fact that we cannot use an expectation operator to value risk and a secure arbitrage-free price. Of course, in the context of pure actuarial risks, many pricing operators called "actuarial premium principles" have been proposed. The following display recalls the three most well-known actuarial principles to price the discounted loss $H$ at maturity; see, e.g., Kaas et al. (2008) for the motivation and details.

$$
\begin{array}{rlrl}
\text { Variance Principle: } & \Pi[H]=\mathbb{E}[H]+\frac{1}{2} \alpha \operatorname{Var}[H], & & \alpha \geq 0 \\
\text { Standard-Deviation Principle: } & \Pi[H]=\mathbb{E}[H]+\beta \sqrt{\operatorname{Var}[H]}, & & \beta \geq 0 \\
\text { Cost-of-Capital Principle: } & \Pi[H]=\mathbb{E}[H]+\delta \operatorname{VaR}_{q}[H-\mathbb{E}[H]], & \delta \geq 0
\end{array}
$$

Most actuarial premium principles, including those previously noted, are a nonlinear function of discounted loss and impose an extra risk premium (also called "Risk Loading") on the "Best-Estimate" of future insurance losses. Both best-estimate and risk loading are calculated under the real-world measure $\mathbb{P}$. This postulates that actuarial pricing is an economic decision under uncertainty, whereas financial pricing is normally based on the risk-neutral valuation built using an equivalent martingale measure that utilizes a conditional expected value under a risk-adjusted underlying process. Goovaerts and Laeven (2008) discussed the no-arbitrage argument for the actuarial principles for pure insurance risks and the securitized version of the insurance products.

In itself, risk loading is another risk measure that plays the role of a buffer to cover the possible deviation of future losses from what is expected from the best-estimate of the losses. In the Variance principle, the loading is a positive proportion of the variance risk measure that applies to the discounted loss, whereas in the Standard-Deviation Principle, the standard deviation of the discounted loss measures the risk premium. Bühlmann (1970) discussed the properties of these two premium principles in detail. In the Cost-of-Capital principle, risk loading is measured using value-at-risk with a probability threshold $q$ (in Solvency II, equal to 0.995), where the unexpected loss is calculated as $\operatorname{VaR}_{q}[H-E(H)]$. A small probability $1-q$ always exists that risk loading (capital buffer) is needed to compensate for the real unexpected risk. Therefore, if the buffer capital is "provided" or "injected" by a third party (e.g., by shareholders of the insurance company), the lender requires a cost of capital $\delta$. The insurer then includes this cost of capital as the 
risk loading in price of the insurance risk to be paid by the policyholder.

As a reasonable pricing method, if we add a pure financial claim to a given general payoff $G\left(x_{T}, y_{T}\right)$, we expect that the pure financial part of the portfolio should be priced consistently with arbitrage-free pricing. Note that, although we assume that the market containing only financial payoffs $G^{S}$ is complete, the market given by general $\mathscr{F}$-measurable payoffs $G$ is incomplete. Moreover, the payoffs replicable by perfectly liquid assets do not carry risks more than the market risk. We formalize this concept in the following definition.

Definition 4.1.1. An actuarial pricing operator $\prod_{g}$ conditional on the actuarial information $\mathscr{G}^{A}$ and the financial information $\mathscr{F}^{S}$ is market-consistent if, for any financial derivative $G^{S}\left(x_{T}\right)$ and any general claim $G\left(x_{T}, y_{T}\right)$, we have

$$
\Pi_{\mathscr{G}}\left(G+G^{S}\right)=\Pi_{\mathscr{G}}[G]+\mathbb{E}_{\mathscr{G}}^{\mathrm{Q}}\left[G^{S}\right]
$$

The market-consistency definition postulates that, given the actuarial information, the actuarial price of a general payoff plus a pure financial payoff equals the actuarial price of the general payoff plus the arbitrage-free price of the pure financial payoff. This establishes a generalized notion of "translation invariance" for the $\mathscr{G}$-conditional valuation operator with respect to the pure financial risk. This implies that if any risk exists that is hedgeable (even in the payoff $G$ ), it must be hedged through a market-consistent valuation. Hence, a market-consistent valuation cannot be improved through hedging. The similar representation of the market-consistency can be found in Kupper et al. (2008), Malamud et al. (2008), or Pelsser and Stadje (2014).

\section{Two-step Actuarial Valuation in One-period Setting}

Pelsser and Stadje (2014) proposed that the market-consistent value of a contingent payoff can be constructed using a two-step market evaluation method. Using this market evaluation method, the price is developed by splitting the no-arbitrage financial price operator, $\mathbb{E}^{\mathrm{Q}}$ (which prices a hedgeable pure financial payoff), and an actuarial premium principle, $\Pi^{\mathbb{P}}$ (which prices a general (partially) unhedgeable payoff). From now on, we call this operator a "two-step actuarial operator", or simply "two-step operator" or "two-step valuation".

In a one-period setting, to value the mixed position $G\left(y_{T}, x_{T}\right)$ at time $t<T$, a conceptual representation of the two-step actuarial operator is defined as follows ${ }^{1}$ :

\footnotetext{
${ }^{1}$ See example (3.4) in Pelsser and Stadje (2014) for examples of two-step actuarial operators under the previously described representation.
} 
Definition 4.1.2. An actuarial premium principle $\prod_{\mathscr{G}}: L^{2}(\mathscr{F}) \rightarrow L^{2}(\mathscr{G})$ is a two-step market evaluation if

$$
\Pi_{t}\left[G\left(y_{T}, x_{T}\right)\right]=\mathbb{E}^{\mathbb{Q}}\left[\Pi^{\mathbb{P}}\left[G\left(y_{T}, x_{T}\right) \mid \sigma\left(\mathscr{G}_{t}^{A}, \mathscr{F}_{T}^{S}\right)\right] \mid \sigma\left(\mathscr{G}_{t}^{A}, \mathscr{F}_{t}^{S}\right)\right] .
$$

This equation formulates the function of the two-step valuation. In the inner step (i.e., the first step of the two-step evaluation), the $\sigma\left(\mathscr{G}_{t}^{A}, \mathscr{F}_{T}^{S}\right)$-conditional actuarial operator $\Pi^{\mathbb{P}}$ is executed, whereas the financial process $x_{T}$ is a $\mathscr{F}_{T}^{S}$-measurable variable. Hence, the only randomness comes from the actuarial risk factor (insurance process) at time $T, y_{T}$, conditional on $\mathscr{G}_{t}^{A}$, and $x_{T}$ stays constant. Let $y_{t}$ and $x_{t}$ be stochastic processes with a Markov property. They exclusively reflect all information available at $\mathscr{G}_{t}^{A}$ and $\mathscr{F}_{t}^{S}$, respectively, and are used instead for conditioning. Applying the inner operator, turns the term $\Pi^{\mathbb{P}}\left[G\left(y_{T}, x_{T}\right) \mid \sigma\left(\mathscr{G}_{t}^{A}, \mathscr{F}_{T}^{S}\right)\right]$ into the function $G^{S}\left(t, T, y_{t}, x_{T}\right)$ which is therefore a random variable measurable with respect to $\sigma\left(\mathscr{G}_{t}^{A}, \mathscr{F}_{T}^{S}\right)$. Now, applying the outer conditional expectation $\mathbb{E}^{\mathrm{Q}}$ with respect to $\sigma\left(\mathscr{G}_{t}^{A}, \mathscr{F}_{t}^{S}\right)$, the only source of randomness in $G^{S}$ is $x_{T}$. This shows that the structure of the two-step actuarial evaluation in (4.1.4) is well defined.

Hence, a simple description of the two-step actuarial valuation in a one-period setting over $[t, T]$ is as follows:

- In the first (inner) step, we assume that we know all financial information up to and including time $T$, whereas our knowledge of actuarial information is up to time $t<T$ (i.e., we know $\left.y_{t}\right)$. We calculate the actuarial value of the payoff $G\left(y_{T}, x_{T}\right)$ under the real-world measure $\mathbb{P}$ given $\left(y_{T}, x_{t}\right)$.

$$
\Pi^{\mathbb{P}}\left[G\left(y_{T}, x_{T}\right) \mid\left(y_{t}, x_{T}\right)\right]
$$

The result of this step turns the general payoff $G\left(y_{T}, x_{T}\right)$ into a function exhibited by $G^{S}\left(y_{t}, x_{T}\right)$.

- Because looking from time $t, G^{S}\left(y_{t}, x_{T}\right)$ is random only on the financial risk $x_{T}$, it can be perfectly hedged given the completeness of the financial market and the noarbitrage argument. Hence, the second (outer) step can be performed through the conditional expectation under the risk-adjusted measure $\mathbb{Q}$, when we condition on $x_{t}$.

$$
\mathbb{E}^{\mathbb{Q}}\left[G^{S}\left(y_{t}, x_{T}\right) \mid\left(y_{t}, x_{t}\right)\right]
$$

Note that, the second step is still implemented given the actuarial information provided by $y_{t}$.

Hence, in the two-step actuarial valuation, we use both sources of information that we have in hand from $x_{T}$ and $y_{T}$. A simplified form of the two-step market valuation for the actuarial operator $\Pi$ can be defined as follows: 
Definition 4.1.3. Let $y_{t}$ and $x_{t}$ be Markov processes with respect to $\mathscr{G}_{t}^{A}$ and $\mathscr{F}_{t}^{S} . A \mathscr{G}_{-}$ conditional premium principle $\Pi$ is a two-step actuarial operator if

$$
\Pi_{t}\left[G\left(y_{T}, x_{T}\right)\right]=\mathbb{E}^{\mathbb{Q}}\left[\Pi^{\mathbb{P}}\left[G\left(y_{T}, x_{T}\right) \mid\left(y_{t}, x_{T}\right)\right] \mid\left(y_{t}, x_{t}\right)\right] .
$$

In the inner step, under the actuarial operator $\Pi$ and conditional on $y_{t}$ and $x_{T}$, the only randomness is through the actuarial risk process at time $T, y_{T}$. Then, applying the outer conditional expectation $\mathbb{E}^{\mathbb{Q}}$ conditional on $x_{t}$, the only source of randomness is $x_{T}$. We emphasize that, at each time step or sub-interval, two valuation operators need to be applied.

A simplified exhibition of the two-step method can be provided through a binomial discretization of the two state variables $x$ and $y$. At a typical time step $(t, t+\Delta t)$, every state $\left(x_{t}, y_{t}\right)$ of the payoff at time $t$ will develop to four different states of the world at time $t+\Delta t$, as follows

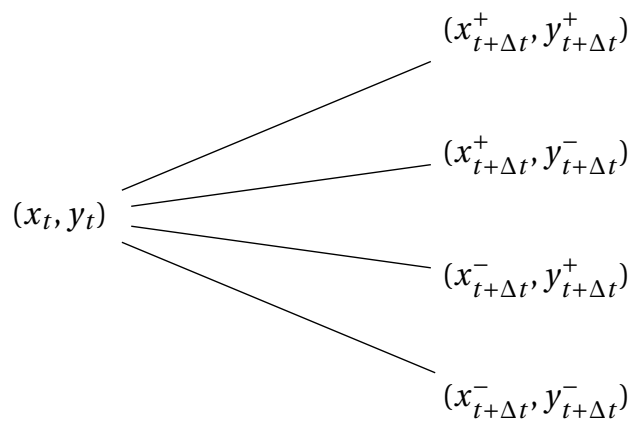

Subsequently, in Section 4.2, we return to this quadrinomial discretization to implement the two-step valuation through the binomial discretization and obtain a continuous-time limit of the two-step valuation. To compute the price, we pretend that, first, $x_{t}$ evolves and ends in two different states at $t+\Delta t$. Only then, given each state of $x_{t+\Delta t}$, does the process $y_{t}$ move. This leads to the following pattern.

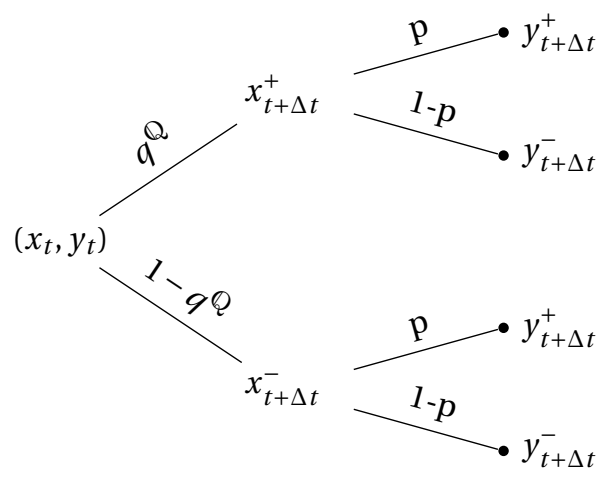


where $q^{\mathbb{Q}}$ is the risk-adjusted ${ }^{2}$ probability of the "up" state for financial risk and $p$ is the probability of the "up" state for actuarial risk. In a two-step valuation, given each state of $x_{t+\Delta t}$ (i.e., we know whether it is $x^{+}$or $x^{-}$) in the inner step (actuarial valuation step), we perform the actuarial valuation for nodes $y_{t+\Delta t}^{+}$and $y_{t+\Delta t}^{-}$. Then, in the outer step (financial valuation step), we have two states of the world that only depend on $x_{t+\Delta t}$, where we compute the price under binomial risk-neutral probabilities $\left(q^{\mathbb{Q}}\right)$.

In a two-step valuation, given each state of $x_{t+\Delta t}$ (i.e., we know whether it is $x^{+}$or $x^{-}$) in the inner step (actuarial valuation step), we perform the actuarial valuation for the nodes

$y_{t+\Delta t}^{+}$and $y_{t+\Delta t}^{-}$. Then, in the outer step (financial valuation step), we have two states of the world that only depend on $x_{t+\Delta t}$, where we compute the price under binomial riskneutral probabilities.

\section{Two-step Actuarial Valuation in Multi-period Setting}

Given the regulatory requirements or for reporting purposes, insurance companies and pension funds need to "re-valuate" their liabilities at regular intervals. Suppose we are at time zero and want to value a contingent payoff at time $T$. At a middle time $0<s<T$, an economic shock, a non-economic decision, or an evolutionary finding can completely change the state and trend of the financial and actuarial risk drivers. We believe that such a condition has a significant effect on the re-valuation of liabilities and must be considered at time zero (present time) in the valuation method. Our aim is to achieve this requirement when constructing the market-consistent actuarial pricing operator.

As previously noted, for long-term liabilities, the one-period setting is not flexible enough to reflect the possible middle-time changes in underlying risk drivers conditional on new information. This setting normally constructs the price by projecting the risk drivers using information at the start time and, accordingly, through determined initial parameter values. In the price/valuation operators, such as the Cost-of-Capital principle that includes the risk measures on a one-year scale, including VaR, this problem becomes highly important.

We reconstruct the two-step actuarial valuation using two different multi-period pricing settings:

- EIOPA pricing framework,

- Time-consistent pricing.

\section{Two-step Actuarial Valuation under EIOPA Standard}

The technical provisions for most insurance liabilities must be valuated as the summation of the best-estimate and the risk-margin. Because the main source of technical pro-

\footnotetext{
${ }^{2}$ Also called the "risk neutral" probability
} 
visions is insurance premiums, this must also be considered in the pricing phase. The premium principles in Eq. (4.1.2) are non-linear examples of these pricing operators in a one-period setting in which risk loading on top of the expected value is the risk-margin for the price. The Technical Specifications for the Preparatory Phase (Part 1) (2014) released by the European Insurance and Occupational Pension Authority (EIOPA) provides advice on the calculation of the risk-margin on top of the best-estimate for long-term liabilities ${ }^{3}$. The risk-margin component is an adjustment to cover the uncertainty arising from the unhedgeable part of liabilities. Unhedgeable risk can be covered by "buffer capital" provided by external stakeholders, such as shareholders of the company. In return, capital providers ask for a cost-of-capital (on top of the risk-free rate) to compensate for their investment in a risky position. Because the risk-margin is measured using a one-year VaR operator, it is consistent with the Cost-of-Capital premium principle for pricing a long-term liability. We use a multi-period pricing operator method. The EIOPA framework advises that the confidence level of the VaR be set to $99.5 \%$ and the cost-of-capital to $6 \%$.

Articles TP.5.5-TP.5.10, TP.5.64, and TP.5.68 of Technical Specifications for the Preparatory Phase (Part 1) (2014) specifically explain the calculation of the risk-margin over the valuation period. Suppose we only have the actuarial risk $y_{t}$ with payoff $f\left(y_{T}\right)$ for $t<T$. The EIOPA risk-margin price at time $t$ for a contract with payoff at time $T>t$, can be formulated by $\Pi_{t}^{E}$ as follows:

$$
\Pi_{t}^{E}\left[f\left(y_{T}\right)\right]=h\left(y_{t}\right)+\delta \sum_{k=1}^{T-t} \operatorname{VaR}_{q}\left[h\left(y_{t+k}\right)-h\left(y_{t}\right) \mid \mathbb{B E}\left(y_{t+k-1}\right)\right],
$$

where for $k=0,1,2, \ldots, T-t$

$$
h\left(y_{t+k}\right)=\mathbb{E}\left[f\left(y_{T}\right) \mid y_{t+k}\right]
$$

is the best-estimate final payoff given the information available at time $t+k$, and

$$
\operatorname{BE}\left(y_{t+k-1}\right)=\mathbb{E}^{\mathbb{P}}\left[y_{t+k-1} \mid y_{t}\right]
$$

shows the best-estimate of $y_{t+k+1}$ given its value at time $t, y_{t} . \Pi_{t}^{E}$ is the conditional Costof-Capital operator with available information at time $t<T$. For now we ignore the discounting, and come back to it later.

The base value of the price is still the one-period best-estimate of the payoff at time $T$ computed by the conditional expectation $\mathbb{E}\left[f\left(y_{T}\right) \mid y_{t}\right]=f\left(y_{t}\right)$. The summation term over annual time-steps from $t+1$ until maturity $T$ can be interpreted as follows. For each time-step ( $s$, we consider the one-year VaR along the best-estimate path of $y$. Hence, the VaR-operator is conditioned on $\operatorname{BE}\left(y_{t+k-1}\right)$. Using this projected value of $y_{t+k-1}$ at each

\footnotetext{
${ }^{3}$ For more general regulations, see the EU Delegated Regulation for the insurance and reinsurance business (Solvency II) in (EC Delegated Regulation 2015/35 (2015))
} 
time step as a starting point, we then consider the impact of a $99.5 \%$ worst-case shock in non-market risks $y_{t+k}$ on the best-estimate of the payoff at time $t+k$, represented by $f\left(y_{t+k}\right)$. This is the projected buffer-capital for time $t+k$. Over this buffer-capital, we must pay the cost-of-capital $\delta$ to capital providers. The discounted sum of all of these cost-of-capital payments is the "EIOPA Risk-margin". Equivalently, this formulates the risk measurement as follows: over the valuation period $[t, T]$ for each point in time $t+k \leq$ $T$, we take the best-estimate value of the underlying risk driver one year earlier $t+k-1$ as the available information. Based on that, we calculate the VaR of the best-estimate of the payoff $f\left(y_{t+k}\right)$ as the risk-margin of that one year. Therefore, when $k=0$, using the information available at $t$, we calculate the VaR of the best-estimate payoff, $f\left(y_{T}\right)$, at time $t+1$. Then, we walk forward where $k=1$ and receive new information at time $t+1$. Therefore, our judgment on the best-estimate payoff can be updated using this new information and we calculate the VaR of the best-estimate payoff at time $t+2$ using the available information at $t+1$, which is $\operatorname{BE}\left(y_{t+1}\right)$. Walking along the best-estimate path of $y$, for each point in time $t+k$, we measure the one-year conditional VaR on the bestestimate payoff at $t+k+1$ until we reach $t+k+1=T$.

Although the EIOPA Risk-margin pricing operator is calculated in a multi-period setting, it is not a time-consistent pricing operator. The EIOPA Risk-margin pricing operator considers the uncertainty arising from non-market risks $y_{T}$ on the best-estimate price. However, there is a "second-order" effect, which is the uncertainty arising from the nonmarket risks $y_{T}$ on future buffer capital. Therefore, EIOPA's Risk-margin pricing operator ignores the "capital-on-capital" effect that a full time-consistent operator considers. The same fact holds for the other risk measures, such as Standard-Deviation and Variance operators. Further reasoning on this can be found in Pelsser (2011). To improve the marketconsistent actuarial price, we include the time-consistency property in the two-step actuarial operator by construction through the backward iteration of the one-period operators explained as follows.

Now, we construct a two-step actuarial valuation in equation (4.1.5) with the EIOPA risk-margin operator $\Pi_{t}^{E}$ in Eq. (4.1.6). The resulting market-consistent actuarial price for a general payoff $G\left(y_{T}, x_{T}\right)$ has the following form:

$$
\Pi_{t}\left[G\left(y_{T}, x_{T}\right)\right]=\mathbb{E}^{\mathbb{Q}}\left[e^{-r(T-t)} \times \Pi_{t}^{E}\left[G\left(y_{T}, x_{T}\right) \mid\left(y_{t}, x_{T}\right)\right] \mid\left(y_{t}, x_{t}\right)\right]
$$

with the inner step operator

$$
\Pi_{t}^{E}\left[G\left(y_{T}, x_{T}\right) \mid\left(y_{t}, x_{T}\right)\right]=h\left(y_{t}, x_{T}\right)+\delta \sum_{k=1}^{T-t} \operatorname{VaR}_{q}^{\mathbb{P}}\left[h\left(y_{t+k}, x_{T}\right)-h\left(y_{t}, x_{T}\right) \mid\left(\operatorname{BE}\left(y_{t+k-1}\right), x_{T}\right)\right]
$$

where for $k=0,1,2, \ldots, T-t$ 


$$
h\left(y_{t+k}, x_{T}\right)=\mathbb{E}^{\mathbb{P}}\left[G\left(y_{T}, x_{T}\right) \mid\left(y_{t+k}, x_{T}\right)\right] .
$$

is the best-estimate final payoff conditioned on the actuarial information at time $t+k$.

In the inner step, we first calculate the best-estimate value of the payoff given the value of the financial risk $x_{T}$ and actuarial risk $y_{t}$. Then, for each year $k$, we add the summation of the one-year VaR values of the best-estimate payoff $h\left(y_{t+k}, x_{T}\right)$, conditional on the known financial state variable $x_{T}$ and the best-estimate value of the underlying actuarial process one year earlier, $\mathrm{BE}\left(y_{t+k-1}\right)$. In fact, at each point in time $t+k$, we look forward to the final payoff and consider its best-estimate given the actuarial information at $t+k$. Each VaR value is basically calculated using a 99.5\% shock on the underlying actuarial risk for one year, given its best-estimate one year earlier, $t+k-1$. The output of the inner step is again, by construction, a payoff that depends only on $x_{T}$ as the source of randomness and is valuated by the conditional expectation under the risk-adjusted measure $\mathbb{Q}$, given $x_{t}$. Note that, $\mathrm{BE}\left(y_{t+k-1}\right)$ is also obtained given $y_{t}$ and, hence, is conditional on the pair $\left(\mathbb{B E}\left(y_{t+k-1}\right), x_{T}\right)$. The conditional expectation $\mathbb{E}^{\mathbb{P}}\left[G\left(y_{T}, x_{T}\right) \mid\left(y_{t}, x_{T}\right)\right]$ can come out of the VaR operator given the translation-invariance property. ${ }^{4}$

\section{Time-Consistent Two-step Actuarial Valuation}

The re-valuation requirement for the insurance liabilities, is consistent with the concept of the time-consistency property of the valuation operator. Under a time-consistent price operator, if position $A$ is riskier than position $B$ at some time in the future, then it is guaranteed to be riskier at any time prior to that point. Therefore, if $\rho_{t}$ denotes the price of $A$ and $B$ at time $t$, under time-consistency for $T>0, \rho_{T}(A)>\rho_{T}(B)$, and then $\forall t<T$, $\rho_{t}(A)>\rho_{t}(B)$. As a result, the value of the time- $T$ liability at time zero should be equal to the price obtained as if we value the liability at a middle time $0<s<T$, and then value the time- $s$ value of the liability at time zero. This can be translated as the following:

Definition 4.1.4. A conditional valuation operator $\left(\Pi_{t}\right)$ is time-consistent if and only if, for all $0 \leq t<s \leq T$ and any measurable non-negative payoff $f(y(T))$,

$$
\Pi_{t}[T, f(y(T))]=\Pi_{t}\left(s, \Pi_{s}[T, f(y(T))]\right) .
$$

This "recursive" form of the time-consistency definition is used to construct the timeconsistent actuarial operator in a multi-period setting. Time-consistency is a generalization of the "no-arbitrage" arguments widely discussed in the financial pricing literature, and is constructed by the "tower property" of the conditional expectation operator, ${ }^{5}$ which can now be extended to non-linear operators. A formal definition of the time-consistency

\footnotetext{
${ }^{4}$ Technically saying that $\mathbb{E}^{\mathbb{P}}\left[G\left(x_{T}, y_{t+k}\right) \mid\left(x_{T}, \mathbb{B E}\left(y_{t+k-1}\right)\right)\right]$ is not $\sigma\left(\mathscr{F}_{T}^{S}, \mathscr{G}_{t+k-1}^{A}\right)$-measurable.

${ }^{5}$ Note that the conditional expectation is time consistent.
} 
can be found in Föllmer and Penner (2006), Cheridito and Stadje (2009), and Acciaio and Penner (2011).

We apply the time-consistency property to the two-step actuarial valuation to expand market-consistency, at least in a finite number of points, over the entire period $[0, T]$ in a dynamic setting. In fact, we are interested in preserving the market-consistency for all possible middle points in the valuation period. This can be achieved by applying the "Backward Iteration" method proposed by Jobert and Rogers (2008) to the two-step operator. If the valuation period $[0, T]$ is divided into a set of sub-intervals, backward iteration constructs a time-consistent valuation by connecting and re-valuating the one-period valuation (in our case, the two-step actuarial valuation) over the sub-intervals in a backward manner, starting from $T$. See, for example, Pelsser and Salahnejhad (2016) for an overview of how time-consistency can be obtained for well-known actuarial premium principles through the backward iteration method. For the two-step actuarial valuation, the idea is generally described as follows.

Suppose we would like to value a time- $T$ payoff at time zero. The representation in equation (4.1.5) does not necessarily imply that the two-step actuarial valuation must be applied in a one-period valuation setting over $(0, T)$. We divide the time interval $[0, T]$ using a discrete set of points $\{0, \Delta t, 2 \Delta t, \ldots, T-\Delta t, T\}$ into a number $n=\frac{T}{\Delta t}$ of sub-intervals of the form $(t, t+\Delta t)$. The backward iteration procedure starts from time $T$ over the last sub-interval $(T-\Delta t, T)$ to value the payoff $G\left(T, x_{T}, y_{T}\right)$ at time $T-\Delta t$. Using the backward iteration method, we calculate the price process of a contract at time $0 \leq t<T$, where the price at $t+\Delta t$ for any $t$ is considered as a new payoff. Let the one-period $\mathscr{G}$-conditional actuarial price at time $T-\Delta t$, be denoted by $\pi(T-\Delta t, x, y)$. Using the two-step actuarial valuation in equation (4.1.5), we have

$$
\pi_{\mathscr{G}}\left(T-\Delta t, x_{T-\Delta t}, y_{T-\Delta t}\right)=\mathbb{E}^{\mathbb{Q}}\left[\Pi^{\mathbb{P}}\left[G\left(T, x_{T}, y_{T}\right) \mid y_{T-\Delta t}, x_{T}\right] \mid y_{T-\Delta t}, x_{T-\Delta t}\right] .
$$

Then, we move one step backward in time and over the sub-interval $(T-2 \Delta t, T-\Delta t)$, we assume $\pi_{\mathscr{G} A}(T-\Delta t, x, y)$ as a new payoff. We apply (4.1.5) again as $\pi_{\mathscr{G}_{A}}\left(T-2 \Delta t, x_{T-2 \Delta t}, y_{T-2 \Delta t}\right)=\mathbb{E}^{\mathbb{Q}}\left[\Pi^{\mathbb{P}}\left(\pi\left(T-\Delta t, x_{T-\Delta t}, y_{T-\Delta t}\right) \mid y_{T-2 \Delta t}, x_{T-\Delta t}\right) \mid y_{T-2 \Delta t}, x_{T-2 \Delta t}\right]$.

We continue this backward iteration of the one-period valuation for all sub-intervals $(t, t+\Delta t)$, to finally reach the time step $(0, \Delta t)$, where we obtain the contract price at time zero. Again note that, we fully apply the two-step valuation in each time step $(t, t+\Delta t)$. The first step for the payoff, conditional on the financial risk, can be considered as a pure insurance risk for the real-world measure $\mathbb{P}$. The second step is an arbitrage-free valuation of the financial derivative in the equivalent martingale measure $\mathbb{Q}$.

The general time-consistent two-step valuation operator for the typical time step $(t, t+$ 
$\Delta t)$ is

$\pi\left(t, x_{t}, y_{t}\right)=\mathbb{E}^{\mathbb{Q}}\left[\Pi^{\mathbb{P}}\left[\pi\left(t+\Delta t, x_{t+\Delta t}, y_{t+\Delta t}\right) \mid y_{t}, x_{t+\Delta t}\right] \mid y_{t}, x_{t}\right]$

with terminal condition

$$
\pi\left(T, x_{T}, y_{T}\right)=G\left(T, x_{T}, y_{T}\right) .
$$

This general representation is of the simultaneous time-consistent and market-consistent "TCMC" actuarial price when, in the general payoff, there is only one univariate state variable for each pair of the financial and actuarial risk. To derive the price in a theoretical setting, one can increase the number of intervals of the form $(t, t+\Delta t)$ in the limit by taking the limit of this valuation equation when $\Delta t \rightarrow 0$. As a result, the price will simultaneously have time-consistency and market-consistency throughout the valuation period $[0, T]$.

The important application point is that, as we mentioned in the introduction and based on Pelsser and Stadje (2014), the market-consistency that resulted by applying the two-step valuation holds even if we apply backward iteration to a finite number of predictable middle points in time over $[0, T]$ without taking the limit for $\Delta t$. Hence, for example, if we are interested in calculating the market-consistent price of a 20 -year unit-linked contract, dividing the period $[0,20]$ into any number of sub-intervals $\left(n=\frac{T}{\Delta t}=1,2,3, \ldots\right.$ ) and applying the two-step valuation preserves the result as a market-consistent price. Note that the price produced from a finite number of sub-intervals in this framework is an approximation of the continuous-time limit of a TCMC price, as previously mentioned.

\section{Time-Consistency Risk Premium}

Different operators may offer different prices for the same contingent payoff. Suppose we exhibit the one-period actuarial value of risk at time $t$ by $\Pi_{t}$, and the time-consistent actuarial valuation driven by the backward iteration by $\Pi_{t}^{\mathrm{TC}}$. Both operators are constructed using the same actuarial premium principle and all other parameters are equal. The possible fundamental difference between these two prices is only the result of enforcing the time-consistency property mentioned in definition 4.1.4. We call this price difference the “Time-Consistency Risk Premium” (TCRP) and show it as

$$
\text { Time-Consistency Risk Premium: } \quad k^{\mathrm{TC}}(t, T)=\Pi_{t}^{\mathrm{TC}}-\Pi_{t}
$$

In case there exists an analytical solution for the time-consistent actuarial valuation (by the continuous-time limit of the time-consistent operator when $\Delta t \rightarrow 0$ ), $k^{\mathrm{TC}}$ can also be obtained analytically. In applications, practitioners use an approximations of the timeconsistent price (e.g., they work with $\Delta t=$ one year) that gives an approximated TCRP: $\hat{k}^{\mathrm{TC}}(t, T)=\Pi_{t}^{\hat{\mathrm{TC}}}-\Pi_{t}$. Clearly, if we calculate the one-period and time-consistent actuarial values under the two-step actuarial valuation operator, then $k^{T C}$ will be obtained for a 
market-consistent price.

\subsection{Two-step Pricing through Binomial Discretization}

We provide a simple illustration of the construction of the two-step actuarial valuation by a binomial discretization of the actuarial and financial processes. Suppose that actuarial risk $y_{t}$ follows the diffusion process

$$
\mathrm{d} y_{t}=a\left(t, y_{t}\right) \mathrm{d} t+b\left(t, y_{t}\right) \mathrm{d} W_{t}^{a}
$$

and financial risk can be modeled in a Black and Scholes (1973) economy with the logarithm of the stock price process $x_{t}=\ln S_{t}$ under a real-world measure $\mathbb{P}$ as follows

$$
\mathrm{d} x_{t}=\left(\mu\left(t, x_{t}\right)-\frac{1}{2} \sigma^{2}\left(t, x_{t}\right)\right) \mathrm{d} t+\sigma\left(t, x_{t}\right) \mathrm{d} W_{t}^{f}
$$

where $W_{t}^{a}$ and $W_{t}^{f}$ are correlated standard Brownian motions through coefficient $\rho$. Because the Black-Scholes economy is complete and arbitrage-free, there exists a unique martingale measure $\mathbb{Q}$ under which $x_{t}$ is given by

$$
\mathrm{d} x_{t}=\left(r-\frac{1}{2} \sigma^{2}\left(t, x_{t}\right)\right) \mathrm{d} t+\sigma\left(t, x_{t}\right) \mathrm{d} W_{t}^{f, \mathbb{Q}}
$$

and the pricing operator is a conditional expectation with adjusted probabilities.

Suppose that both underlying processes, $y_{t}$ and $x_{t}$, in (4.2.1) and (4.2.2), are discretized under a real-world measure $\mathbb{P}$ through a binomial tree with "up" and "down" probabilities equal to $p=1 / 2$. However, under measure $\mathbb{Q}$, we should discretize $x_{t}$ using adjusted probabilities such that the drift in the process becomes equal to $\left(r-\frac{1}{2} \sigma^{2}(t, x)\right)$. Hence, $y_{t}$ and $x_{t}$ will be discretized during period $(t, t+\Delta t)$ as follows

$$
\begin{aligned}
& y_{t+\Delta t}=y_{t}+a \Delta t+\left\{\begin{array}{lll}
+b \sqrt{\Delta t} & \text { with P-prob. } & \frac{1}{2} \\
-b \sqrt{\Delta t} & \text { with P-prob. } \frac{1}{2}
\end{array}\right. \\
& x_{t+\Delta t}=x_{t}+\left(\mu-\frac{1}{2} \sigma^{2}\right) \Delta t+\left\{\begin{array}{lll}
+\sigma \sqrt{\Delta t} & \text { with } \mathbb{Q} \text {-prob. } & \frac{1}{2}\left(1-\frac{\mu-r}{\sigma} \sqrt{\Delta t}\right) \\
-\sigma \sqrt{\Delta t} & \text { with } \mathbb{Q} \text {-prob. } & \frac{1}{2}\left(1+\frac{\mu-r}{\sigma} \sqrt{\Delta t}\right)
\end{array}\right.
\end{aligned}
$$

where we assume that $a, b, \mu$, and $\sigma$ are constant. The quantity $(\mu-r) / \sigma$ that modifies the probabilities from $\mathbb{P}$ to $\mathbb{Q}$ is the Radon-Nikodym exponent of $\mathrm{dQ} / \mathrm{dP}$ and is called the "market-price of financial risk."

The two processes $x_{t}$ and $y_{t}$ can be jointly discretized in a "Quadrinomial" tree under the real-world measure $\mathbb{P}$ as illustrated in Figure 4.1. The joint probabilities are computed such that the correlation between processes $x$ and $y$ equals $\rho$, and the marginal proba- 


\begin{tabular}{|c|c|c|}
\hline \multirow[b]{2}{*}{ State: } & \multirow{2}{*}{\multicolumn{2}{|c|}{$y_{t+\Delta t}$}} \\
\hline & & \\
\hline \multirow{2}{*}{$x+$} & $(\underline{1+\rho})$ & $(\underline{1-\rho})$ \\
\hline & 4 & 4 \\
\hline$x_{t+\Delta t}$ & & \\
\hline \multirow{2}{*}{$x-$} & $(\underline{1-\rho})$ & $(1+\rho)$ \\
\hline & 4 & 4 \\
\hline
\end{tabular}

Figure 4.1: Joint probabilities of the Quadrinomial discretization for $x_{t}$ and $y_{t}$.

bilities for each individual process equal the binomial $\mathbb{P}$-probabilities of $1 / 2$. The signs "+" and "-" in front of $x$ and $y$ represent the "up" and "down" states on the binomial tree, respectively.

Given this quadrinomial discretization, we seek to obtain the market-consistent Variance price of the general position $G\left(T, x_{T}, y_{T}\right)$ using the two-step valuation. In a multiperiod setting under the time-consistency argument, we use the equation (4.1.11) and substitute for the Variance principle from (4.1.2a). We use $\pi(t+\Delta t)$ instead of $\pi(t+$ $\left.\Delta t, x_{t+\Delta t}, y_{t+\Delta t}\right)$ to shorten the notation. Hence, the time-consistent two-step Variance operator is

$$
\pi\left(t, y_{t}, x_{t}\right)=e^{-r \Delta t} \mathbb{E}^{\mathrm{Q}}\left[\mathbb{E}^{\mathbb{P}}\left[\pi(t+\Delta t) \mid y_{t}, x_{t+\Delta t}\right]+\frac{1}{2} \alpha \operatorname{Var}^{\mathbb{P}}\left[\pi(t+\Delta t) \mid y_{t}, x_{t+\Delta t}\right] \mid y_{t}, x_{t}\right]
$$

with $\pi(T)=G\left(T, x_{T}, y_{T}\right)$ as the terminal condition. Looking ahead from time $t$, we have four possible outcomes for $\pi(t+\Delta t)=\left\{\pi_{++}, \pi_{+_{-}}, \pi_{-+}, \pi_{--}\right\}$. In this shorthand notation, the first and second subscripts denote the state of the financial and actuarial processes, respectively.

The two-step valuation procedure is as follows.

- First (Inner) step: Suppose we know that the financial process at $t+\Delta t$ can be two possible states, $x+$ or $x-$. Conditional on the value $x_{t+\Delta t}$, only pure insurance risk remains, which we can price using the Variance principle. Hence, in the one-period quadrinomial tree, conditional on $x+$ or $x$-, (as the inner step) we can explicitly calculate the Variance price of the $\pi(t+\Delta t)$ as follows

$$
\pi^{\mathrm{v}}(t+\Delta t \mid x \pm):=\mathbb{E}\left[\pi^{\mathrm{v}}(t+\Delta t) \mid x \pm\right]+\frac{1}{2} \frac{\gamma}{X_{0} e^{r(t+\Delta t)}} \operatorname{Var}\left[\pi^{\mathrm{v}}(t+\Delta t) \mid x \pm\right]
$$


with

$$
\begin{aligned}
\mathbb{E}\left[\pi^{\mathrm{v}}(t+\Delta t) \mid x+\right] & =\left(\frac{1+\rho}{2}\right) \pi_{++}^{\mathrm{v}}+\left(\frac{1-\rho}{2}\right) \pi_{+-}^{\mathrm{v}} \\
\mathbb{E}\left[\pi^{\mathrm{v}}(t+\Delta t) \mid x-\right] & =\left(\frac{1-\rho}{2}\right) \pi_{-+}^{\mathrm{v}}+\left(\frac{1+\rho}{2}\right) \pi_{--}^{\mathrm{v}} \\
\operatorname{Var}\left[\pi^{\mathrm{v}}(t+\Delta t) \mid x+\right] & =\left(\frac{1-\rho^{2}}{4}\right)\left(\pi_{++}^{\mathrm{v}}-\pi_{+-}^{\mathrm{v}}\right)^{2} \\
\operatorname{Var}\left[\pi^{\mathrm{v}}(t+\Delta t) \mid x-\right] & =\left(\frac{1-\rho^{2}}{4}\right)\left(\pi_{-+}^{\mathrm{v}}-\pi_{--}^{\mathrm{v}}\right)^{2} .
\end{aligned}
$$

As a result, $\pi^{\mathrm{v}}(t+\Delta t \mid x \pm)$ is a payoff only depending on financial states $x \pm$. Note that in the inner step, we are still acting on the real-world probability $\mathbb{P}$ that allows us to use the quadrinomial probabilities in Figure 4.1. In fact, in the first step, we used the "pure actuarial" Variance principle to find the Variance price (at time $t+\Delta t$ ) of the insurance risk, conditional on the realization of the financial risk.

- Second (Outer) step: $\pi^{\mathrm{v}}(t+\Delta t \mid x \pm)$ is now a derivative only depending on the hedgeable financial risk $x$ with binomial realizations $x \pm$. Hence, in the second step, we do the arbitrage-free valuation for the binomial tree as

$$
\begin{aligned}
\pi^{\mathrm{V}}\left(t, x_{t}, y_{t}\right) & =e^{-r \Delta t} \mathbb{E}^{\mathrm{Q}}\left[\pi^{\mathrm{V}}(t+\Delta t \mid x \pm)\right] \\
& =e^{-r \Delta t}\left(\frac{1}{2}\left(1-\frac{\mu-r}{\sigma} \sqrt{\Delta t}\right) \pi^{\mathrm{V}}(t+\Delta t \mid x+)+\frac{1}{2}\left(1+\frac{\mu-r}{\sigma} \sqrt{\Delta t}\right) \pi^{\mathrm{V}}(t+\Delta t \mid x-)\right)
\end{aligned}
$$

where we used adjusted probabilities under the $\mathbb{Q}$ measure in Equation (4.2.4b).

Note that, in the first step, for $\rho=1$ or $\rho=-1$, the financial and insurance markets become perfectly correlated and, in these cases, the conditional variance goes to zero. A similar approach can be applied to Standard-Deviation and Cost-of-Capital principles in (4.1.2), as well as the other well-known actuarial premium principles.

\section{Continuous-time Limit of the Market-Consistent Variance Price}

We are interested in the continuous-time limit of the two-step actuarial valuation when $\Delta t \rightarrow 0$ for all sub-intervals of the form $(t, t+\Delta t)$ during the valuation period $[0, T]$. Theoretically, this means that we repeat the backward iteration infinitely many times, starting from $T$ and ending at $t=0$.

Suppose in Eq. (4.1.11) that $\pi\left(t+\Delta t, x_{t+\Delta t}, y_{t+\Delta t}\right)$ is twice continuously differentiable with respect to $x$ and $y$. The Taylor expansion of $\pi$ around the point $\left(y_{t}, x_{t}\right)$ can be written 
as

$$
\begin{aligned}
\pi\left(t+\Delta t, y_{t}+h_{y}, x_{t}+h_{x}\right)=\pi\left(t+\Delta t, y_{t}, x_{t}\right)+h_{y} \pi_{y}\left(t+\Delta t, y_{t}, x_{t}\right)+h_{x} \pi_{x}\left(t+\Delta t, y_{t}, x_{t}\right) \\
+\frac{1}{2} h_{y}^{2} \pi_{y y}\left(t+\Delta t, y_{t}, x_{t}\right)+\frac{1}{2} h_{x}^{2} \pi_{x x}\left(t+\Delta t, y_{t}, x_{t}\right)+h_{x} h_{y} \pi_{x y}\left(t+\Delta t, y_{t}, x_{t}\right)
\end{aligned}
$$

where $h_{y}=a \Delta t \pm b \sqrt{\Delta t}$ and $h_{x}=\left(\mu-\frac{1}{2} \sigma^{2}\right) \Delta t \pm \sigma \sqrt{\Delta t}$ are binomial random increments with realizations $\left\{h_{y+}, h_{y-}\right\}$ and $\left\{h_{x+}, h_{x_{-}}\right\}$respectively. In the notations, (only) subscripts on $\pi$ denote partial derivatives. Depending on the values of $h_{y}$ and $h_{x}$, the Taylor expansion basically calculates one of the four states $\left\{\pi_{++}, \pi_{+-}, \pi_{-+}, \pi_{--}\right\}$at time $t+\Delta t$. For example,

$$
\pi_{+-}=\pi\left(t+\Delta t, y_{t}+h_{y-}, x_{t}+h_{x+}\right)
$$

In the inner step in Eq. (4.2.6), conditional on the state of $x$, we substitute the Taylor expansion in (4.2.8) for each corresponding state of $\pi\left(t+\Delta t, y_{t}+h_{y}, x_{t}+h_{x}\right)$. Substituting for all four states of $\pi$ at time $t+\Delta t$ in (4.2.6) and gathering all terms in ascending order of $\Delta t$, we obtain

$$
\begin{aligned}
\pi^{\mathrm{v}}(t+\Delta t \mid x+) & =\pi^{\mathrm{v}}\left(t+\Delta t, y_{t}, x_{t}\right)+\left[\sigma \pi_{x}^{\mathrm{v}}+\rho b \pi_{y}^{\mathrm{v}}\right] \sqrt{\Delta t} \\
& +\left[a \pi_{y}^{\mathrm{v}}+\left(\mu-\frac{\sigma^{2}}{2}\right) \pi_{x}^{\mathrm{v}}+\frac{b^{2}}{2} \pi_{y y}^{\mathrm{v}}+\frac{\sigma^{2}}{2} \pi_{x x}^{\mathrm{v}}+\rho b \sigma \pi_{x y}^{\mathrm{v}}+\frac{\alpha b^{2}}{2}\left(1-\rho^{2}\right)\left(\pi_{y}^{\mathrm{v}}\right)^{2}\right] \Delta t \\
& +\mathscr{O}(\Delta t \sqrt{\Delta t}) \\
\pi^{\mathrm{v}}(t+\Delta t \mid x-) & =\pi^{\mathrm{v}}\left(t+\Delta t, y_{t}, x_{t}\right)-\left[\sigma \pi_{x}^{\mathrm{v}}+\rho b \pi_{y}^{\mathrm{v}}\right] \sqrt{\Delta t} \\
& +\left[a \pi_{y}^{\mathrm{v}}+\left(\mu-\frac{\sigma^{2}}{2}\right) \pi_{x}^{\mathrm{v}}+\frac{b^{2}}{2} \pi_{y y}^{\mathrm{v}}+\frac{\sigma^{2}}{2} \pi_{x x}^{\mathrm{v}}+\rho b \sigma \pi_{x y}^{\mathrm{v}}+\frac{\alpha b^{2}}{2}\left(1-\rho^{2}\right)\left(\pi_{y}^{\mathrm{v}}\right)^{2}\right] \Delta t \\
& +\mathscr{O}(\Delta t \sqrt{\Delta t})
\end{aligned}
$$

where all derivative terms are functions of $\left(t+\Delta t, y_{t}, x_{t}\right)$, which we suppress to shorten the notation. Note that this conditional expression of $\pi$ is random with respect to the $\sqrt{\Delta t}$ term.

In the outer step, we substitute these polynomials in Eq. (4.2.7) and again rearrange the $\Delta t$ terms in ascending order. With some simplification, we obtain

$$
\begin{aligned}
& e^{r \Delta t} \pi^{\mathrm{v}}\left(t, y_{t}, x_{t}\right)=\pi^{\mathrm{v}}\left(t+\Delta t, y_{t}, x_{t}\right) \\
+ & {\left[\left(a-\rho b \frac{\mu-r}{\sigma}\right) \pi_{y}^{\mathrm{v}}+\left(r-\frac{\sigma^{2}}{2}\right) \pi_{x}^{\mathrm{v}}+\frac{b^{2}}{2} \pi_{y y}^{\mathrm{v}}+\frac{\sigma^{2}}{2} \pi_{x x}^{\mathrm{v}}+\rho b \sigma \pi_{x y}^{\mathrm{v}}+\frac{\alpha b^{2}}{2}\left(1-\rho^{2}\right)\left(\pi_{y}^{\mathrm{v}}\right)^{2}\right] \Delta t+\mathscr{O}(\Delta t \sqrt{\Delta t}) . }
\end{aligned}
$$


We use the Taylor series of $e^{r \Delta t}=1+r \Delta t+\mathscr{O}\left(\Delta t^{2}\right)$ and divide both sides by $\Delta t$

$$
\begin{aligned}
& \frac{\pi^{\mathrm{v}}\left(t, y_{t}, x_{t}\right)-\pi^{\mathrm{v}}\left(t+\Delta t, y_{t}, x_{t}\right)}{\Delta t}+r \pi^{\mathrm{v}}\left(t, y_{t}, x_{t}\right)= \\
& \left(a-\rho b \frac{\mu-r}{\sigma}\right) \pi_{y}^{\mathrm{v}}+\left(r-\frac{\sigma^{2}}{2}\right) \pi_{x}^{\mathrm{v}}+\frac{b^{2}}{2} \pi_{y y}^{\mathrm{v}}+\frac{\sigma^{2}}{2} \pi_{x x}^{\mathrm{v}}+\rho b \sigma \pi_{x y}^{\mathrm{v}}+\frac{\alpha b^{2}}{2}\left(1-\rho^{2}\right)\left(\pi_{y}^{\mathrm{v}}\right)^{2}+\mathscr{O}(\sqrt{\Delta t}) .
\end{aligned}
$$

If we take the limit when $\Delta t \rightarrow 0$, the fraction on the left-hand side converges to the derivative of $\pi^{\mathrm{v}}$ with respect to $t$ (i.e., $-\pi_{t}^{\mathrm{v}}\left(t, x_{t}, y_{t}\right)$ ). Hence, we obtain the following partial differential equation (pde) as a representation of the continuous-time limit of the TCMC Variance price

$\pi_{t}^{\mathrm{v}}+\left(a-\rho b \frac{\mu-r}{\sigma}\right) \pi_{y}^{\mathrm{v}}+\left(r-\frac{1}{2} \sigma^{2}\right) \pi_{x}^{\mathrm{v}}+\frac{1}{2} b^{2} \pi_{y y}^{\mathrm{v}}+\frac{\sigma^{2}}{2} \pi_{x x}^{\mathrm{v}}+\rho b \sigma \pi_{x y}^{\mathrm{v}}+\frac{1}{2} \frac{\gamma}{X_{0} e^{r t}}\left(1-\rho^{2}\right)\left(b \pi_{y}^{\mathrm{v}}\right)^{2}-r \pi^{\mathrm{v}}=0$

where we suppressed (again) the dependence of the $\pi$ terms to $\left(t, y_{t}, x_{t}\right)$ to lighten the notation, and substituted for $\alpha=\gamma / X_{0} e^{r t}$.

This pde clearly reflects the characteristics of the market-consistency and its impact on price $\pi$. The pde shows that the financial risk process $x_{t}$ has an arbitrage-free drift term $\left(r-\frac{1}{2} \sigma^{2}\right)$ from the measure $\mathbb{Q}$. The actuarial process $y_{t}$ is evaluated using the adjusted drift term, where $\rho b(\mu-r) / \sigma$ deducted from $a$ can be interpreted as a partial hedge cost that affects the price. The partial hedge cost is a proportion of the correlated part of the diffusion coefficient $b$, where the ratio is the market price of financial risk, $(\mu-r) / \sigma$. This is consistent with change of the measure to $\mathbb{Q}$, for the "financial" Brownian motion $W^{f}$. Furthermore, a non-linear term is added that reflects the "local unhedgeable variance" $\left(1-\rho^{2}\right)\left(b \pi_{y}^{\mathrm{v}}\right)^{2}$ of actuarial risk. The rest of the pds terms correspond to the elements of the Feynman-Kac pde for a linear price operator. Unfortunately, there is no explicit solution for the non-linear pde in (4.2.12), which calls for numerical methods to provide the solution.

As a special case, suppose the payoff has no explicit dependence on financial risk $x$ (while still present in the market); however, the actuarial risk through $\rho \neq 0$ is connected to the market index with a constant market price of risk, $(\mu-r) / \sigma$. The pde in Eq. (4.2.12) will be modified as

$$
\pi_{t}^{\mathrm{v}}+\left(a-\rho b \frac{\mu-r}{\sigma}\right) \pi_{y}^{\mathrm{v}}+\frac{1}{2} b^{2} \pi_{y y}^{\mathrm{v}}+\frac{1}{2} \frac{\gamma}{X_{0} e^{r t}}\left(1-\rho^{2}\right)\left(b \pi_{y}^{\mathrm{v}}\right)^{2}-r \pi^{\mathrm{v}}=0
$$

The pde shows that, through correlation to the financial market, we can still hedge part of the actuarial risk. Note that, in the previous special case, the market with actuarial risk is incomplete and there is no martingale representation for the price of the risk. Hence, 
instead of $\pi_{x}$, holding the $\rho b \pi_{y} / \sigma$ proportion of $x$ can hedge the position.

By using the log-transformation $\exp \left(\frac{\gamma}{X_{0} e^{r t}} \pi^{\mathrm{v}}(t, y)\right)$, the pde in Eq. (4.2.13) can be solved as follows

$$
\pi^{\mathrm{V}}(t, y)=\frac{X_{0} e^{r t}}{\gamma\left(1-\rho^{2}\right)} \ln \mathbb{E}^{\mathbb{V}}\left[e^{\frac{\gamma\left(1-\rho^{2}\right)}{X_{0} e^{r t}} f\left(y_{T}\right)} \mid y_{t}=y\right]
$$

where $\mathbb{E}^{\mathbb{V}}$ is the expectation under the risk-adjusted measure for $y_{t}$ with the modified drift rate. Derivation of this solution is provided in Pelsser and Salahnejhad (2016). Henderson (2002) and Musiela and Zariphopoulou (004b) also derived this solution for the exponential indifference pricing.

\section{Continuous-time Limit of the Market-consistent Standard-Deviation Price}

Suppose that instead of the Variance price, we use the Standard-Deviation premium principle. Hence, in the inner step, the first part of Eq. (4.2.6) is modified as follows

$$
\pi^{\mathrm{v}}(t+\Delta t \mid x \pm):=\mathbb{E}\left[\pi^{\mathrm{v}}(t+\Delta t) \mid x \pm\right]+\beta \sqrt{\Delta t} \sqrt{\operatorname{Var}\left[\pi^{\mathrm{v}}(t+\Delta t) \mid x \pm\right]}
$$

and the remaining part calculates the conditional expectations, and the variances remain the same. Note that the factor $\sqrt{\Delta t}$ is multiplied by the standard deviation to solve the dimensionality problem that arises from the different time scales produced by expectations and the standard deviation. For more details on this, see Pelsser and Salahnejhad (2016).

We repeat the same method by substituting the Taylor expansion of $\pi\left(t+\Delta t, y_{t}+\right.$ $\left.h_{y}, x_{t}+h_{x}\right)$ in Eq. (4.2.8) into the outer step of the valuation in Eq. (4.2.7). We follow the same procedure from Eqs. (4.2.9)-(4.2.11) and take the limit when $\Delta t \rightarrow 0$. We obtain the following pde for the Standard-Deviation premium principle,

$$
\pi_{t}^{\mathrm{v}}+\left(a-\rho b \frac{\mu-r}{\sigma} \pm b \beta \sqrt{1-\rho^{2}}\right) \pi_{y}^{\mathrm{v}}+\left(r-\frac{1}{2} \sigma^{2}\right) \pi_{x}^{\mathrm{v}}+\frac{b^{2}}{2} \pi_{y y}^{\mathrm{v}}+\frac{\sigma^{2}}{2} \pi_{x x}^{\mathrm{v}}+\rho b \sigma \pi_{x y}^{\mathrm{v}}-r \pi^{\mathrm{v}}=0
$$

where the sign of $\pm b \beta \sqrt{1-\rho^{2}}$ is given by the sign of $\pi_{y}$. By construction, this pde is the continuous-time limit of the TCMC Standard-Deviation price. Although we changed the pricing operator, the drift term of process $x,\left(r-\sigma^{2} / 2\right)$, shows that the financial part is still priced under the no-arbitrage argument using the two-step valuation. Compared with the Variance price in the previous section, the actuarial risk $y_{t}$ is priced differently when there is no more local unhedgeable variance. Instead, market-consistency affects the actuarial risk through two different modifications in the drift term. The first is the same hedge cost in the Variance price, $\rho b \frac{\mu-r}{\sigma}$, which partially hedges the actuarial risk through its connection to the market price of the risk. Moreover, there is an "upwind" risk adjustment $\pm b \beta \sqrt{1-\rho^{2}}$ that is a proportion of the actuarial diffusion $b$ and increases the price to a 
higher and more prudential level (from the insurer's point of view) than the real-world measure $\mathbb{P}$. Note that, if payoff $f\left(x_{T}, y_{T}\right)$ is a monotonically increasing function of $y_{T}$, the drift is adjusted upward through $+b \beta \sqrt{1-\rho^{2}}$, and downward through $-b \beta \sqrt{1-\rho^{2}}$ if the payoff is a monotonically decreasing function of $y_{T}$.

The pde for Standard-Deviation price corresponds to the two-dimensional form of the Feynman-Kac formula with the solution

$$
\pi^{\mathrm{V}}\left(t, x_{t}, y_{t}\right)=\mathbb{E}^{\mathbb{S}}\left[e^{-r(T-t)} f\left(x_{T}, y_{T}\right)\right]
$$

The solution represents that the continuous-time limit of the TCMC Standard-Deviation price of the mixed financial and actuarial payoff is a linear operator under the new riskadjusted measure $\mathbb{S}$. This measure modifies the drift of the both financial and the actuarial processes exhibited in Eq. (4.2.16). 



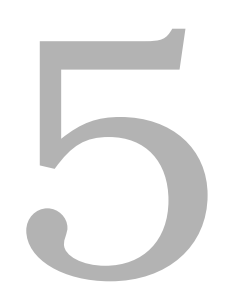

\section{Market-Consistent Pricing for Unit-linked \\ Contract}

\section{This chapter is based on:}

Salahnejhad Ghalehjooghi, A. and Pelsser, A., (2016), Market-Consistent Actuarial Valuations with Applications to EIOPA Risk-margin and Time-consistent Pricing, under review in "Scandinavian Actuarial Journal".

In this chapter we implement the two-step actuarial operator for a simple unit-linked contract with the equity and mortality, respectively as the underlying financial and actuarial risk drivers. We provide some insights into formulation of the EIOPA risk-margin and timeconsistent price with the two-step actuarial operator under independence assumption. To perform the numerical calculations we adapt a customized version of the Least Square Monte Carlo (LSMC) method and simulate the three market-consistent prices (the two above and the best-estimate price) that we discussed in the previous chapter. We show that the two-step actuarial valuation captures partial (or perfect) hedging as we observe that all three prices converge to one adjusted Best-Estimate price when the correlation between financial and actuarial risks increases. 


\subsection{Market-Consistent Valuation for Unit-linked Contract}

We consider a simple unit-linked contract for a cohort of policyholders with age $x$. Each policyholder participates in the contract by buying a unit of it at start time $t=0$. We assume that the contract has no guarantee. We define the payoff as a factorized combination of the financial and actuarial risks

$$
G\left(S_{T}, T_{x}\right)=S_{T} \times N_{x}(T)
$$

where $T$ is the maturity time, $S_{T}$ is the market value of the investment asset made by the premiums, and $N_{x}(T)$ is the number of survivors of the original cohort who reach age $x+T$. We assume that the cohort $N_{x}$ is known at the start of the contract. At maturity, the market value of the investment asset will be paid to survivors. If $N_{x}(T)=\mathbb{1}_{T_{x} \geq T}$, the contract is specialized for an individual of age $x$, with the remaining lifetime random variable $T_{x}$. Normally, in the unit-linked contracts, $T$ is the retirement age minus age ${ }^{1}$.

To model the payoff and implement the two-step actuarial valuation, we need to model both financial risk $S_{t}$ and actuarial risks $T_{x}$.

\section{Risk Drivers}

In the payoff function in Eq. (5.1.1), $S_{T}$ is a financial risky asset following Geometric Brownian Motion (GBM)

$$
d S_{t}=\mu_{S} S_{t} d t+\sigma_{S} S_{t} d W_{t}^{S}
$$

Here, $\mu_{S}$ and $\sigma_{S}$ are constant and $W_{t}^{S}$ is a standard Brownian motion defined on filtration $\mathscr{F}_{t} S$ on the time interval $[0, T]$ under the real-world measure $\mathbb{P}$. In the complete market, under the unique equivalent martingale measure $\mathbb{Q}$ and a constant continuously compounded interest rate $r, S_{t}$ follows

$$
d S_{t}=r S_{t} \mathrm{~d} t+\sigma_{S} S_{t} \mathrm{~d} \tilde{W}_{t}^{S}
$$

with the solution

$$
S_{t}=S_{0} \exp \left(\left(r-\frac{1}{2} \sigma_{S}^{2}\right) t+\sigma_{S} \tilde{W}_{t}^{S}\right)
$$

where $S_{0}$ is the initial value of the investment and $\tilde{W}_{t}^{S}$ is the $\mathbb{Q}$-standard Brownian motion.

For the actuarial risk, we start by defining some notations. We denote the survival and death probability of $(x)$ using ${ }_{t} p_{x}=\mathbb{P}\left(T_{x}>t\right)$ and ${ }_{t} q_{x}=1-{ }_{t} p_{x}$, respectively. The forceof-mortality of the individual aged $x$ at time $t$ is defined as $\mu(x+t)=-\frac{d}{d t} \ln \left({ }_{t} p_{x}\right)^{2}$.

\footnotetext{
${ }^{1}$ For this case, we may assume that $T=70-x$

${ }^{2}$ Considering the stochastic evolution of mortality risk through time, a more precise concept is "the remaining lifetime at the beginning of the calendar year $t$ " for which the notation is $T_{x}(t)$.
} 
Through dynamic mortality, we assume that ${ }_{t} p_{x}$ is random in any future time $t$ and, effectively, $\mu(x+t)$ as an index of mortality risk follows a stochastic process. To model the evolution of the survival probability and to consider the projection of the future mortality/longevity index, we use the model introduced by Lee and Carter (1992). In the LeeCarter model, for an integer age $x$ and calendar year $t$, the force-of-mortality (intentionally denoted using different notation comparing to static force-of-mortality), $\mu_{x}(t)$, is assumed to be given by

$$
\ln \mu_{x}(t)=\alpha_{x}+\beta_{x} \kappa_{t}
$$

where $\kappa_{t}$ is the general level of mortality, $\alpha_{x}$ is the average age-specific mortality, and $\beta_{x}$ is the age-specific sensitivity of the mortality to a change in $\kappa_{t}$. Given an alternative point of view, $\kappa_{t}$ can be interpreted as a latent process to model the longevity trend, specified by $\kappa_{0}=0$ and

$$
\mathrm{d} \kappa_{t}=\mu_{\kappa} \mathrm{d} t+\sigma_{\kappa} \mathrm{d} W_{t}^{\kappa}
$$

with the solution $\kappa_{t}=\kappa_{0}+\mu_{\kappa} t+\sigma_{\kappa} W_{t}^{\kappa}$ and $W^{\kappa}$ a standard Brownian motion under measure $\mathbb{P}^{3}$ The best-estimate for $\kappa_{t}$ process at time zero is $\mathbb{E}\left(\kappa_{t}\right)=\kappa_{0}+\mu_{\kappa} t$.

The general procedure for using the Lee-Carter model starts by choosing the realized mortality rates of the past calendar years. The process continues with calibrating the parameters of the stochastic mortality model, $\alpha_{x}$ and $\beta_{x}$. It is noteworthy to mention that, in the notation, if we assume the present $t=0$, the notation $t$ is representative of the past "calendar times" $\left\{t_{0}, t_{0}+1, \ldots, 0\right\}$ in the model. Once the age-specified Lee-Carter parameters are estimated, the future force-of-mortality can be projected for individual $(x)$. In that sense, $t>0$ is the notation of the future time. In this chapter, we assume that we have the estimated parameters of the Lee-Carter model through the most recent realizations of the mortality rates in the Netherlands. ${ }^{4}$ We then concentrate on the simulation of the future mortality rates.

Conditional on $\kappa_{t}$, the remaining lifetime the policyholders are assumed to be independent. Moreover, the force-of-mortality of an individual of age $x+t$ at future time $t$ is given by

$$
\begin{aligned}
\mu_{x}(t) & =\exp \left(\alpha(x+t)+\beta(x+t) \kappa_{t}\right) \\
& =\exp \left(\alpha(x+t)+\beta(x+t) \mu_{\kappa} t+\beta(x+t) \sigma_{\kappa} W_{t}^{\kappa}\right)
\end{aligned}
$$

Note that the parameters $\alpha, \beta: \mathbb{R}_{+} \rightarrow \mathbb{R}$ are assumed to be piecewise constant on the intervals $[t, t+1)$. This means that for all $u \in[0,1), \mu_{x+u}(t+u)=\mu_{x}(t)$. Because we may valuate

\footnotetext{
${ }^{3}$ Note that $\kappa$ can also be modeled as follows
}

$$
\kappa_{t_{k+1}}=\mu_{\kappa}+\kappa_{t_{k}}+\varepsilon_{t_{k}: t_{k+1}},
$$

with $\varepsilon_{t_{k}}: t_{k+1}$ i.i.d. $N\left(0, \sigma_{\kappa}^{2}\right)$, which is the time-series model used in Lee and Carter (1992).

${ }^{4}$ We use the mortality rates between 1960-2006 for males and females. 
the price of a product for a group of participants, let $N_{x}(t)$ denote the cohort of policyholders of age $x+t$ at time $t$. We assume that the death events of the group members are independent. Then, conditional on $\kappa_{t}$ and $N_{x}(t), N_{x}(t+1)$ has a binomial distribution with parameters $N_{x}(t)$ and success probability

$$
\exp \left(-\mu_{x}(t)\right)=\exp \left(-\exp \left(\alpha(x+t)+\beta(x+t)\left(\kappa_{t}\right)\right)\right)
$$

Note that, $\kappa_{t}$ is the underlying process of the remaining lifetime random variable $T_{x}$. Moreover, for the Lee-carter model, we define actuarial filtration as $\mathscr{G}_{t}^{A}=$ $\sigma\left(\left\{T_{x} \leq s\right\}, \kappa_{s}, s \leq t\right)$, where $\kappa_{s}$ is part of the information set. We only focus on systemic risk, where the idiosyncratic mortality risk should be a second-order effect compared with the effect of a longevity trend. This assumption could be motivated by observing the realized mortality tables previously mentioned.

In the next two subsections, we implement the market-consistent price of the payoff in Eq. (5.1.1) using the two-step actuarial valuation under EIOPA risk-margin and timeconsistent pricing operators. As is shown, the formulation only depends on the form of the payoff function, rather than the risk drivers.

\section{Market-Consistent Price under the EIOPA Standard}

We apply the two-step EIOPA operator in Eqs. (4.1.7) and (4.1.8) to the unit-linked payoff in Eq. (5.1.1). The market-consistent EIOPA risk-margin price at time $t<T$ for a cohort of age $x$ is as follows:

$$
\Pi_{\mathscr{G}_{t}}\left[S_{T}\right]=e^{-r(T-t)} \mathbb{E}^{\mathrm{Q}}\left[\Pi_{t}^{E}\left[S_{T} \times N_{x}(T) \mid S_{T}, \kappa_{t}\right] \mid S_{t}, \kappa_{t}\right]
$$

where

$$
\begin{aligned}
\Pi_{t}^{E}\left[S_{T} \times N_{x}(T) \mid\left(S_{T}, \kappa_{t}\right)\right]=\mathbb{E}^{\mathbb{P}}\left[S_{T} \times N_{x}(T) \mid S_{T}, \kappa_{t}\right] \\
\quad+\delta \sum_{k=1}^{T-t} \operatorname{VaR}_{q}^{\mathbb{P}}\left[f\left(S_{T}, N_{x}(k)\right)-\mathbb{E}^{\mathbb{P}}\left[S_{T} \times N_{x}(T) \mid S_{T}, \kappa_{t}\right] \mid S_{T}, \operatorname{BE}\left(\kappa_{t+k-1}\right)\right] .
\end{aligned}
$$

and that

$$
f\left(S_{T}, N_{x}(k)\right)=\mathbb{E}^{\mathbb{P}}\left[S_{T} \times N_{x}(T) \mid S_{T}, \kappa_{t+k}\right]
$$

is the best-estimate of the final payoff at $T$, given the actuarial information at time $t+k$. As we always consider the final payoff estimation, the discount factor for all summation terms is still $e^{-r(T-t)}$ and can be applied in the outer step. The formula asserts the fact that, to valuate any risk measure depending on the survival event at time $t+k$, we have full information about the longevity trend before and including the earlier time at $t+k-1$. 


\section{Formulation under Independence}

Suppose at time $t=0$, when the value of the underlying process $\kappa_{0}$ is known, we would like to price a contract with maturity $T$ for an individual aged $x$ instead of the cohort. Under the independence assumption, the operator in equation (5.1.9) can be factorized for the financial risk driver $S_{T}$ and simplified as follows

$$
\begin{aligned}
\Pi_{\mathscr{G}_{0}^{A}}^{E}\left[S_{T} \mathbb{1}_{T_{x}>T}\right] & =e^{-r T} \mathbb{E}^{\mathrm{Q}}\left[S_{T} \mid S_{0}\right] \times \\
& {\left[\mathbb{E}^{\mathbb{P}}\left[\mathbb{1}_{\left\{T_{x}>T\right\}} \mid \kappa_{0}\right]+\delta \sum_{k=1}^{T} \operatorname{VaR}_{q}^{\mathbb{P}}\left[\mathbb{E}^{\mathbb{P}}\left[\mathbb{1}_{\left\{T_{x}>T\right\}} \mid \kappa_{k}\right]-\mathbb{E}^{\mathbb{P}}\left[\mathbb{1}_{\left\{T_{x}>T\right\}} \mid \kappa_{0}\right] \mid \operatorname{BE}\left(\kappa_{k-1}\right)\right]\right] } \\
& =S_{0} \times\left[{ }_{T} \bar{p}_{x}+\delta \sum_{k=1}^{T}\left(\operatorname{VaR}_{q}^{\mathbb{P}}\left[\mathbb{E}^{\mathbb{P}}\left[\mathbb{1}_{\left\{T_{x}>T\right\}} \mid \kappa_{k}\right] \mid B E\left(\kappa_{k-1}\right)\right]-{ }_{T} \bar{p}_{x}\right)\right] \\
& =S_{0} \times\left[{ }_{T} \bar{p}_{x}+\delta \sum_{k=1}^{T}\left(\operatorname{VaR}_{q}^{\mathbb{P}}\left[{ }_{k} \bar{p}_{x} \times{ }_{T-k} p_{x+k}-{ }_{T} \bar{p}_{x}\right]\right)\right] \\
& =S_{0} \times\left[{ }_{T} \bar{p}_{x}+\delta \sum_{k=1}^{T}{ }_{k} \bar{p}_{x} \times\left(\operatorname{VaR}_{q}^{\mathbb{P}}\left[{ }_{T-k} p_{x+k}\right]-{ }_{T-k} \bar{p}_{x+k}\right)\right] .
\end{aligned}
$$

where $"{ }_{t} \bar{p}_{x}$ " denotes the deterministic value of the survival probability when " ${ }_{t} p_{x}$ " is its stochastic version.

In the second equation, $S_{0}$ is obtained according to the martingale property for $S_{T}$ under the risk-neutral measure $\mathbb{Q}$. Moreover, the expectation $\mathbb{E}^{\mathbb{P}}\left[\mathbb{1}_{\left\{T_{x}>T\right\}} \mid \kappa_{0}\right]$ is obviously equal to the deterministic value of the survival probability ${ }_{T} \bar{p}_{x}$. Note that, given $B E\left(\kappa_{k-1}\right)$, ${ }_{T} \bar{p}_{x}$ can come out of the VaR operator given the translation invariance property. Standing at $t=0$, the conditional expected value $\mathbb{E}^{\mathbb{P}}\left[\mathbb{1}_{\left\{T_{x}>T\right\}} \mid \kappa_{k}\right]$ under the VaR operator is a random survival probability. However, conditioning on $\mathbb{B E}\left(\kappa_{k-1}\right)$ means that we have part of the information on $\kappa_{t}$, up to and including $k-1$. Knowing $\kappa_{k}$ also imposes that, given that an individual aged $x$ is alive at time $k$ (with probability ${ }_{k} \bar{p}_{x}$ ), the random part is the survival probability of an individual aged $x+k$ up to $T-k$ years. Therefore, the term under VaR can be written as the product ${ }_{k} \bar{p}_{x} \times{ }_{T-k} p_{x+k}$ in the third equality. The fourth equality can be obtained by the product rule for probabilities and the scale invariance property of VaR.

In fact, this conditional VaR represents an imaginary situation as if we only follow the best-estimate path for $\kappa_{t}$ until $\mathrm{BE}\left(\kappa_{k-1}\right)$ and look into the horizon of the final payoff and measure a one-year risk on the expectation of the payoff. The EIOPA risk-margin price suggests that the difference between the 99.5\%-quantile of the survival probability until maturity and the average survival probability is the annual risk margin, and considers the summation of these differences during the valuation period as the risk loading for the actuarial price. Note that, in general, we do not know the distribution of the conditional 
payoff " $\mathbb{1}_{\left\{T_{x}>T\right\}} \mid \kappa_{k}$ " to calculate the quantile. However, using the Lee-Carter model, we can always simulate future mortality/longevity functions and calculate it numerically.

\section{Time-consistent Market-consistent Price}

Now, we turn to TCMC valuations of the simple unit-linked contract. For every time step $(t, t+\Delta t)$, we perform the backward iteration of the valuation in Eq. (4.1.11), whereas the procedure starts from maturity time $T$ by Eq. (4.1.10). Although it is practical to take $\Delta t=1$ (1-year period) in applied situations, we need a benchmark that can be obtained through the analytical solution of the price in continuous-time to check the result of the numerical procedure.

\section{Analytical Solution under Independence}

The analytical solution will be a continuous-time limit of the time-consistent two-step actuarial valuation in (4.1.11) when $\Delta t \rightarrow 0$. We use the independent $S_{t}$ and $\kappa_{t}$ to separate the financial and actuarial operators as $\pi_{T-\Delta t}=e^{-r \Delta t} \mathbb{E}^{\mathbb{Q}}\left[S_{T} \mid S_{T-\Delta t}\right] \times \Pi^{\mathbb{P}}\left[f\left(\kappa_{T}\right) \mid \kappa_{T-\Delta t}\right]$ to obtain the actuarial price at time $T-\Delta t$. Then, for the period $(T-2 \Delta t, T-\Delta t)$, we apply the TCMC operator in (4.1.11) as follows

$$
\begin{aligned}
\pi_{T-2 \Delta t} & =e^{-r \Delta t} \mathbb{E}^{\mathbb{Q}}\left[\Pi^{\mathbb{P}}\left[\pi_{T-\Delta t} \mid \kappa_{T-2 \Delta t}, S_{T-\Delta t}\right] \mid S_{T-2 \Delta t}\right] \\
& =e^{-r \Delta t} \mathbb{E}^{\mathbb{Q}}\left[\Pi^{\mathbb{P}}\left[e^{-r \Delta t} \mathbb{E}^{\mathbb{Q}}\left[S_{T} \mid S_{T-\Delta t}\right] \times \Pi^{\mathbb{P}}\left[f\left(\kappa_{T}\right) \mid \kappa_{T-\Delta t}\right] \mid \kappa_{T-2 \Delta t}, S_{T-\Delta t}\right] \mid S_{T-2 \Delta t}\right] \\
& =e^{-r \Delta t} \mathbb{E}^{\mathbb{Q}}\left[e^{-r \Delta t} \mathbb{E}^{\mathbb{Q}}\left[S_{T} \mid S_{T-\Delta t}\right] \times \Pi^{\mathbb{P}}\left[\Pi^{\mathbb{P}}\left[f\left(\kappa_{T}\right) \mid \kappa_{T-\Delta t}\right] \mid \kappa_{T-2 \Delta t}\right] \mid S_{T-2 \Delta t}\right] \\
& =e^{-2 r \Delta t} \mathbb{E}^{\mathbb{Q}}\left[\mathbb{E}^{\mathbb{Q}}\left[S_{T} \mid S_{T-\Delta t}\right] \mid S_{T-2 \Delta t}\right] \times \Pi^{\mathbb{P}}\left[\Pi^{\mathbb{P}}\left[f\left(\kappa_{T}\right) \mid \kappa_{T-\Delta t}\right] \mid \kappa_{T-2 \Delta t}\right] \\
& =S_{T-2 \Delta t} \Pi^{\mathbb{P}}\left[\Pi^{\mathbb{P}}\left[f\left(\kappa_{T}\right) \mid \kappa_{T-\Delta t}\right] \mid \kappa_{T-2 \Delta t}\right]
\end{aligned}
$$

where the third equality obtained as $\mathbb{E}^{\mathrm{Q}}\left[S_{T} \mid S_{T-\Delta t}\right]$ is $S_{T-\Delta t}$-measurable, the fourth equality is provided attributable to independence, and the last equality is clear from the tower property and the martingale property of $S_{t}$ under the risk-neutral measure $\mathbb{Q}$.

If we repeat this valuation procedure in a backward manner for all time intervals $(t, t+\Delta t)$ in $[0, T]$ ending in $t=0$ and take the limit when $\Delta t \rightarrow 0$, we obtain the analytical solution of the TCMC actuarial valuation in (5.1.11). Pelsser and Salahnejhad (2016) showed that, in continuous-time when $\Delta t \rightarrow 0$, for some well-known actuarial premium principles $\Pi$, the value of the nested actuarial operator

$$
\pi(t, \kappa(t))=\Pi^{\mathbb{P}}\left[\Pi^{\mathbb{P}}\left[f\left(\kappa_{t+2 \Delta t}\right) \mid \kappa_{t+\Delta t}\right] \mid \kappa_{t}\right]
$$

has an analytical solution. They showed that for the Cost-of-Capital price operator, $\Pi^{c}=$ $\mathbb{E}\left[f\left(\kappa_{T}\right) \mid \kappa_{t}\right]+\delta \sqrt{T-t} \operatorname{VaR}_{q}\left[f\left(\kappa_{T}\right)-\mathbb{E}\left[f\left(\kappa_{T}\right)\right] \mid \kappa_{t}\right]$, the analytical solution of $\pi\left(t, \kappa_{t}\right)$ is as 
follows

$$
\pi^{\mathrm{c}}(t, \kappa(t))=\mathbb{E}^{\mathbb{C}}\left[f\left(\kappa_{T}\right) \mid \kappa_{t}\right]
$$

where $\mathbb{E}^{\mathbb{C}}\left[. \mid \kappa_{t}\right]$ denotes the conditional expectation at time $t$ with respect to the "riskadjusted" process $\kappa^{\mathbb{C}}$ defined as

$$
d \kappa_{t}^{\mathbb{C}}=\left(\mu_{\kappa} \pm \delta k \sigma_{\kappa}\right) d t+\sigma_{\kappa} d W_{t}^{\mathbb{C}}
$$

and $k=\Phi^{-1}(1-q)$ for standard normal distribution function $\Phi$.

Having this theoretical result, the continuous-time limit of the two-step Cost-ofCapital price in equation (5.1.11) at time zero, $\pi_{0}^{\mathrm{c}}$, for the payoff function $S_{T} \times f\left(\kappa_{T}\right)=$ $S_{T} \times \mathbb{1}_{\left\{T_{x}>T\right\}}$ will be

$$
\pi_{0}^{\mathrm{c}}=S_{0} \times \mathbb{E}^{\mathbb{C}}\left[\mathbb{1}_{\left\{T_{x}>T\right\}} \mid \kappa_{0}\right]=S_{0} \times{ }_{T} p_{x}{ }^{\mathbb{C}}
$$

where ${ }_{T} p_{x}{ }^{\mathbb{C}}$ is the survival probability of an individual aged $x$ for $T$ years later.

Now, consistent with the "actuarial prudence", we can give a more explicit representation of the risk-adjusted $\kappa^{\mathbb{C}}$ process in (5.1.15). As the payoff $f\left(\kappa_{t}\right)=\mathbb{1}_{\left\{T_{x}>t\right\}}$ and $\Pi^{c}$ are both monotonic decreasing functions of the underlying process $\kappa_{t}$, the risk-adjusted process for $\kappa_{t}$ will be as follows,

$$
d \kappa_{t}^{\mathbb{C}}=\left(\mu_{\kappa}-\delta k \sigma_{\kappa}\right) d t+\sigma_{\kappa} d W_{t}^{\mathbb{C}} .
$$

where $-\delta k \sigma_{\kappa}$ is a negative value and shifts down the mortality trend $\left(\kappa_{t}\right)$ which means that an insurer who is worried about people living longer than expected, will measure higher survival probabilities for. This is consistent with the idea behind the risk-loading in actuarial pricing for the naturally prudent insurer.

$\pi^{\mathrm{c}}$ is, by construction, a TCMC analog of the one-period market-consistent Cost-ofCapital price $\pi_{0}$ for the simple unit-linked contract.

Moreover, Pelsser and Salahnejhad (2016) proved that if, instead of the Cost-of-capital price, we use the Standard-Deviation actuarial principle of the form

$$
\Pi^{s}=\mathbb{E}\left[f\left(\kappa_{T}\right) \mid \kappa_{t}\right]+\beta \sqrt{T-t} \sqrt{\operatorname{Var}\left[f\left(\kappa_{T}\right) \mid \kappa_{t}\right]},
$$

the TCMC Standard-Deviation price $\pi^{\mathrm{s}}$ will converge to the same limit as that of the Costof-Capital price $\pi^{\mathrm{c}}$ in (5.1.14)

$$
\pi_{0}^{\mathrm{s}}=S_{0} \times{ }_{T} p_{x}{ }^{\mathrm{S}}
$$

whereas a slightly different risk-adjusted process for the underlying risk driver $\kappa_{t}^{\mathbb{S}}$ is as follows

$$
d \kappa_{t}^{\mathbb{S}}=\left(\mu_{\kappa}-\beta \sigma_{\kappa}\right) d t+\sigma_{\kappa} d W_{t}^{\mathbb{S}} .
$$


and $-\beta \sigma_{\kappa}$ is again consistent with the actuarial prudence in pricing.

This implies that, for independent actuarial and financial risks, we can use the Standard-Deviation price instead of the market-consistent Cost-of-Capital price of the products similar to unit-linked contract, with factorized payoff structure.

The most impressive result is that the TCMC actuarial price achieved by both the Costof-Capital and the Standard-Deviation premium principle in (5.1.14) and (5.1.16) correspond to the "best-estimate" price of the unit-linked contract in (5.1.1), where the underlying actuarial risk process, $\kappa_{t}$, needs to be adjusted through (5.1.15) or (5.1.17). The risk adjustment of the longevity trend $\kappa_{t}$ intuitively implies the concept of "actuarial prudence" for pricing, where the premium offered by the insurer uses an adjusted drift to make risk loading on top of the expected loss as a more conservative assessment of risk. According to the risk-adjusted process in (5.1.17), when the payoff $f(\kappa)$ is monotonically increasing at $\kappa$, the drift rate is adjusted upwards $\left(\mu_{\kappa}+\beta \sigma_{\kappa}\right)$. For a negative $\mu_{\kappa}$, this will offer a more expensive price for products containing a survival benefit. The opposite occurs when $f(\kappa)$ monotonically decreases at $\kappa$, adjusting the drift downward $\left(\mu_{\kappa}-\beta \sigma_{\kappa}\right)$ and, accordingly, offering a higher price for the payoff that promises to pay on the event of death. Therefore, the risk is always adjusted upward, providing a market-consistent price that is higher than the real-world expected value $\mathbb{E}^{\mathbb{P}}[f(\kappa)]$.

\section{Numerical Results}

In this section, we provide and compare the numerical methodology and results of the market-consistent valuation for the simple unit-linked contract under EIOPA risk-margin and time consistent price.

\section{Numerical Method}

Within this paper, we discussed four different market-consistent prices (using the twostep operator):

1. Best-estimate (Discounted expected) value

2. One-period actuarial price

3. EIOPA actuarial price

4. Time-consistent actuarial price.

The first two prices are in the one-period setting and the second two are constructed in the multi-period setting. The numerical method to conduct the one-period valuation is standard and straightforward, and we only focus on the EIOPA and time-consistent actuarial 
prices. Given the multi-period setting and the conditional structure of the two-step valuation, we must repeat numerous conditional valuation operators for each middle time-step given the state of the underlying processes in previous steps. Theoretically, this means that to perform the calculations at each time step, for every scenario at time $t-1$, we need enough new scenarios at time $t$ to calculate the conditional values. This is translated to build a nested structure of projections for each risk driver involved in the payoff.

We consider a unit-linked product with 30 years to maturity. Suppose to implement the backward iterations and repeat the two-step valuation on an annual basis, we start the simulation at time zero with only three scenarios for each underlying processes $\kappa_{t}$ and $S_{t}$. If we naively apply a nested simulation of the underlying processes at each time step, the calculation will explode. After 30 steps, we have $3^{30}=2.059 \times 10^{14}$ scenarios for each process, if we start the calculation from the maturity time. Note that if $S_{T}$ and $\kappa_{T}$ are dependent, in the first step, we have to make $\left(3^{30}\right)^{2}=4.24 \times 10^{28}$ calculations. We must also repeat the valuation in the backward iteration procedure that creates a higher number of calculations. For the EIOPA price, as we move only on the best-estimate scenario of the underlying risk driver, the previously noted number decreases. However, we still must repeat the projections and calculations for different time steps. Note that for the EIOPA price, as we have only one best-estimate realization to estimate the value, using only three scenarios is inadequate and we must work with much more scenarios. For a long-term valuation period, this scheme is not efficient to execute the calculations in application.

Another technique for addressing this type of valuation is constructing a Markov grid using the finite difference interval. An efficient special case of this method can be implemented by constructing a "recombining trinomial tree." We discretize both $S-T$ and $\kappa_{T}$ into a finite number of states at maturity, where they are both located in their special boundary region. Each state at time $t$ will be connected through three nodes to three different states one step earlier, $t-1$. Equivalently, each state at time $t-1$ is connected to three states at time $t$, which makes it possible to calculate the conditional operator for them at time $t-1$ given this state at $t-1$. Suppose we divide each underlying process into 1,000 states (i.e., scenarios) at time $T$. Applying the two-step valuation for the first time step $(T-1, T)$, for each state of $S_{T}$ in an outer step (i.e., assuming $S_{T}$ known), we have 1,000 trinomial computations of the inner actuarial operator. Hence, moving backward over 30 years, for each time step we repeat $10^{6}$ computations, which accumulates to $3 \times 10^{7}$. Perhaps in a low dimension such as a two-factor model, the finite difference method can achieve the result in a reasonable time. However, in a higher dimension in which an application must work with a portfolio of assets and liabilities, the calculation is not feasible.

To decrease the calculation volume and, in particular, address the extra calculations in the backward iteration method, a useful method is the "Least-Square Monte-Carlo" (LSMC) method. This method is also called "Regression Now", as it uses regression mod- 
els. LSMC was proposed and used by Carriere (1996) and Longstaff and Schwartz (2001) to price different types of American options. The method is widely used in the dynamic valuation of the contingent claims and payoffs using path-dependent risk drivers. LSMC postulates that the conditional expectation of the payoffs can be calculated using the crosssectional information of the underlying risk drivers (i.e., state variables).

To price the unit-linked contract using LSMC, we expect that the conditional expectation of any general payoff $G\left(T, S_{T}, \kappa_{T}\right)$ can be obtained through a series of basis functions of $S_{T}$ and $\kappa_{T}$ as

$$
\mathbb{E}\left[G\left(T, S_{T}, \kappa_{T}\right) \mid \kappa_{t}, S_{t}\right]=f\left(S_{T}, \kappa_{T}\right)=\sum_{k=0}^{\infty} a_{k} e_{k}\left(\kappa_{t}, S_{t}\right)
$$

where $e_{k}(x)$ denotes different types of basis functions, such as

- Polynomials: $1, x, x^{2}, \ldots$

- Fourier basis: $1, \cos (x), \cos (2 x), \ldots$.

The target function $f\left(S_{T}, \kappa_{T}\right)$ at (5.1.18) can be approximated through a finite number of terms in the series that turns it into a regression line

$$
f\left(S_{T}, \kappa_{T}\right) \approx f_{k}\left(S_{T}, \kappa_{T}\right)=\sum_{k=0}^{K-1} a_{k} e_{k}\left(\kappa_{t}, S_{t}\right) .
$$

The fitted value is an estimation of the conditional expectation and can be used to accurately estimate conditional expectations for different forms of $G$.

\section{Approximation of the Conditional VaR in the EIOPA Price}

We turn to the calculation of the EIOPA risk-margin price. Recalling Eq. (5.1.9), the EIOPA price contains a series of one-year conditional VaR given information one year earlier. Under each VaR operator, there is also a conditional expectation of the payoff, $f\left(S_{T}, N_{x}(k)\right)=\mathbb{E}^{\mathbb{P}}\left[S_{T} \times N_{x}(T) \mid S_{T}, N_{t+k}\right]$, given the risk at time $t<T$. Because we do not know the distribution of the survival probability (and, accordingly, the number of survivors $N_{x}(t)$ ), we also do not know the theoretical value of the quantile. To numerically calculate the conditional VaR, the natural solution is to "re-simulate" the underlying risk driver from the state given. For the EIOPA price, this means that in Eq. (5.1.9), we should renew the simulation of $\kappa_{t+k}$ and the number of survivors for every $\mathbb{B E}\left(\kappa_{t+k-1}\right)$, and then simply calculate the appropriate quantile. Doing so imposes a high simulation process load that affects the efficiency of the numerical scheme. The same fact holds for the conditional expectation $f\left(S_{T}, N_{x}(k)\right)$.

A more efficient calculation of these conditional operators in the EIOPA price can be executed using the LSMC method. We start with estimating $f$ in Eq. (5.1.9b) using the 
LSMC method. We simulate $N$ scenarios of the pair $\left(S_{t}, \kappa_{t}\right)$ over the finite number of points for $t=1,2, \ldots, T$. At time $T$, we calculate the payoff $S_{T} \times N_{x}(T)$ (alternatively, $S_{T} \times \mathbb{1}_{T_{x}>T}$ for an individual policy). Note that in the Lee-Carter model, $N_{x}(T)$ is not only a function of $\kappa_{T}$ but also a function of all previous values of $\kappa_{t}$ for $t \leq T$. Therefore, at each point in time $t$, conditioning on $N_{x}(t-1)$ (which contains information on all $\kappa$ values up to and including $t-1)$ as an underlying risk driver is more accurate than conditioning on $\kappa_{t-1}$. Therefore, for every $k=\{1,2, \ldots, T\}$, we obtain an estimation of the conditional expected payoff $f$ by using $N_{x}(k)$ in the basis function instead of $\kappa_{k}$, as follows

$$
\widehat{f}\left(S_{T}, N_{x}(k)\right)=\widehat{\mathbb{E}}^{\mathbb{P}}\left[S_{T} \times N_{x}(T) \mid S_{T}, \kappa_{1}, \kappa_{2}, \ldots, \kappa_{k}\right]=\sum_{m=0}^{M-1}{\widehat{a_{m}}}^{\{k\}} e_{m}\left(S_{T}, N_{x}(k)\right)
$$

where, given $N$ scenario sequences of the underlying risk drivers, the coefficient vector $\widehat{\vec{a}}^{\{k\}}$ is estimated under the least square argument as follows

$$
\widehat{\vec{a}}^{\{k\}}=\underset{\vec{a}^{\{k\}}}{\arg \min } \sum_{i=1}^{N}\left[\left(G_{i}\left(S_{T}, N_{x}(T)\right)-\sum_{k=0}^{K-1} a_{m}^{\{k\}} e_{m}\left(S_{T}(i), N_{x}^{(i)}(k)\right)\right)^{2}\right]
$$

where $G_{i}, S_{T}(i)$, and $N_{x}^{(i)}(k)$, respectively, are the $i$ th realizations of payoff $G$ and state variables $S_{T}$ and $N_{x}(k)$.

To calculate the conditional VaR, one can assume that for a short period (in our case, a one-year horizon), the number of survivors $N_{x}(t)$ is normally distributed. Thus, its conditional $q$-quantile can be approximated by its mean and standard deviation. Regarding each $k$, the $q$ quantile must be conditioned on the best-estimate of $\kappa_{k-1}$. We should calculate the standard deviation of $\left(N_{x}(k) \mid \mathrm{BEE}\left(N_{x} k-1\right)\right)$. We compute the LSMC estimators of the conditional mean and second moment of $N_{x}(k)$ as follows

$$
\begin{array}{ll}
E 1: & \widehat{\mathbb{E}}^{\mathbb{P}}\left[N_{x}(k) \mid N_{x}(k-1)\right]=\sum_{m=0}^{M-1}{\widehat{b_{m}}}^{\{1, k\}} e_{m}\left(N_{x}(k-1)\right) \\
E 2: & \widehat{\mathbb{E}}^{\mathbb{P}}\left[N_{x}(k)^{2} \mid N_{x}(k-1)\right]=\sum_{m=0}^{M-1}{\widehat{b_{m}}}^{\{2, k\}} e_{m}\left(N_{x}(k-1)\right)
\end{array}
$$

which gives the conditional standard deviation Std-Dev $\left(N_{x}(k) \mid \mathbb{B E}\left(N_{x}(k-1)\right)\right)=$ $\sqrt{E 2-(E 1)^{2}}$. Finally, based on the value in Eq. (5.1.9b), the estimation of the conditional $99.5 \%$-quantile is

$$
\begin{aligned}
& \widehat{\operatorname{VaR}}_{99.5 \%}^{\mathbb{P}}\left[f\left(S_{T}, N_{x}(k)\right)-\mathbb{E}^{\mathbb{P}}\left[S_{T} \times N_{x}(T) \mid S_{T}, N_{x}(0)\right] \mid S_{T}, \operatorname{BE}\left(N_{x}(k-1)\right)\right] \\
& \quad=\widehat{f}\left(S_{T}, \operatorname{BE}\left(N_{x}(k)\right)+2.58 \times \operatorname{Std}-\operatorname{Dev}\left(N_{x}(k) \mid \operatorname{BE}\left(N_{x}(k-1)\right)\right)\right)-\widehat{f}\left(S_{T}, \operatorname{BE}\left(N_{x}(k)\right)\right)
\end{aligned}
$$


where $2.58=\Phi^{-1}(99.5 \%)$. This formula measures the difference between the conditional expected payoff at time $k$ when we give a $99.5 \%$ shock to the underlying actuarial driver (here, $N_{x}(k)$ ) and the same value without a shock. The shock gives reference to the bestestimate of the actuarial driver one year earlier, at $k-1$ or $\mathrm{BE}\left(N_{x}(k-1)\right)$. By construction, this value is an estimation of the conditional value-at-risk for the risk margin in year $k$. Obviously, we use Eq. (5.1.9b) to calculate the summation of these values for each $k$ over the valuation period as the total EIOPA risk margin. Finally, for each year $k$, we take the average over the $S_{T}$ values conditional on $S_{0}$. This is the financial mean under the $\mathbb{Q}$ measure and is counted as the outer step in the two-step valuation.

\section{Time-consistent valuation by LSMC}

We explain the implementation of the LSMC method to calculate the time-consistent price of the unit-linked contract. To compare the EIOPA price, we consider the same risk-margin calculation for the unit-linked contract, for which we repeat the conditional one-period two-step actuarial valuation. This valuation consists of the one-year expected value plus the one-year conditional VaR risk margin for each year in a backward procedure. ${ }^{5}$ The one-year conditional VaR is previously calculated in Eq. (5.1.22) for each $k=\{1,2, \ldots, T\}$.

The numerical procedure to calculate the two-step actuarial valuation using LSMC and to implement the backward iteration is as follows.

1. Start with the time step $(T-1, T)$, apply the regressions for the first/inner step, and estimate the regression coefficients $a_{k}^{(1, T)}$ as follows ${ }^{6}$

$$
\widehat{\mathbb{E}}^{\mathbb{P}}\left[S_{T} \times N_{x}(T) \mid S_{T}, \kappa_{1}, \kappa_{2}, \ldots, \kappa_{T-1}\right]=\sum_{k=0}^{K-1}{\widehat{a_{k}}}^{(1, T)} e_{k}\left(S_{T}, N_{x}(T-1)\right)
$$

2. Recalling Eq. (5.1.9b), add the one-year risk-margin estimation that we calculated in Eq. (5.1.22) for year $k=T$ to this conditional expected value and calculate the discounted conditional premium that depends on $N_{x}(T-1)$ and $S_{T}$ as follows

$$
\begin{aligned}
& \pi^{\mathrm{s}}\left(S_{T}, N_{x}(T-1)\right)=e^{-r \Delta t} \times \\
& {\left[\widehat{\mathbb{E}^{\mathbb{P}}}\left[S_{T} \times N_{x}(T)\right]+\delta \widehat{\operatorname{VaR}}_{99.5 \%}^{\mathbb{P}}\left[f\left(S_{T}, N_{x}(k)\right)-\mathbb{E}^{\mathbb{P}}\left[S_{T} \times N_{x}(T) \mid S_{T}, N_{x}(0)\right] \mid S_{T}, \mathbb{B E}\left(N_{x}(k-1)\right)\right]\right] .}
\end{aligned}
$$

\footnotetext{
${ }^{5}$ Note that this time-consistent price is not an analogue for the Cost-of-Capital and/or the Standard-Deviation price.

${ }^{6}$ Using only the last available $\kappa_{t-1}$ will result in the following alternative estimations for Eqs. (5.1.24).
}

$$
\widehat{\mathbb{E}}^{\mathbb{P}}\left[S_{T} \times N_{x}(T) \mid S_{T}, \kappa_{T-\Delta t}\right]=\sum_{k=0}^{K-1}{\widehat{a_{k}}}^{(1, T)} e_{k}\left(S_{T}, \kappa_{T-\Delta t}\right)
$$


Given the actuarial information at time $T-1$ and the financial information at time $T$, this is the conditional actuarial value of the payoff in the inner step at time $T-1$.

3. Apply the regression for the second/outer step

$$
\pi^{\mathrm{s}}\left(S_{T-1}, N_{x}(T-1)\right)=\widehat{\mathbb{E}^{\mathrm{Q}}}\left[\pi^{\mathrm{s}}\left(S_{T}, N_{x}(T-1)\right) \mid S_{T-1}, \kappa-1, \kappa_{2}, \ldots, \kappa_{T-1}\right]=\sum_{k=0}^{K-1} \widehat{b}_{k}^{(T)} e_{k}\left(S_{T-1}\right)
$$

where $\pi^{\mathrm{s}}\left(S_{T-1}, N_{x}(T-1)\right)$ is the value obtained from the outer step, provided in the form of a new $N \times 1$ vector of the payoff assigned to time $T-1$ that will be used to perform a similar two-step valuation, one step backward in $(T-2, T-1)$.

4. Repeat the calculation of the one-year expected value and risk margin in steps 2-4 for all time steps of the form $(k, k+1)$ in a backward manner to reach time zero. ${ }^{7}$

For each time step, the EIOPA price is calculated using three regressions: two regressions for the conditional standard deviation Std-Dev $\left(N_{x}(k) \mid \mathbb{B E}\left(N_{x}(k-1)\right)\right.$ ) (Eqs. (5.1.21a)-(5.1.21b)) and one regression for the conditional expected payoff in the inner step (Eq. (5.1.19)). ${ }^{8}$ The time-consistent price contains five regressions for each one-year time step: three regressions that are the same as the EIOPA one-year risk margin, one regression for the conditional expected value (Eq. (5.1.24)), and one regression for the expectation under $\mathbb{Q}$ in the outer step (Eq. (5.1.26)). Therefore, for a $T$-year contract, we need $3 T$ regressions for EIOPA and $5 T$ regressions for the time-consistent price to run. ${ }^{9}$

For independent financial and actuarial risks, the numerical implementation can be shortened for the time-consistent price by using the analytical results. For example, the value of $S_{T}$ at time $t$ can be factorized from the two-step valuation and then the regression is only needed for the actuarial risk drivers in the inner step.

\section{Results}

The one-period actuarial value and the EIOPA price are not time consistent. Thus, we do not recommend them as proper values in the market-consistent pricing frame. However, we provide them to compare with the time-consistent price and measure the TCRP and the loading we need on top of the EIOPA price to achieve time consistency. The discounted expected value by the tower property is always time consistent and market consistent. Thus, another interesting comparison for practitioners is that between the best-estimate value

\footnotetext{
${ }^{7}$ Technically, in the last period, we have only a single value of $N_{x}(0)$. Thus, instead of the regression in equation (5.1.26), the price is only an actuarial value (mean + standard deviation loading) over the $\pi^{\mathrm{s}}\left(S_{1}, \kappa_{0}\right)$ vector.

${ }^{8}$ The outer step is calculated by taking a simple average over $S_{T}$.

${ }^{9}$ Clearly, if we calculate both prices together, only the two extra regressions are needed for the time-consistent price.
} 
and the time-consistent actuarial price that shows the degree to which the risk loading in the premium principle adds to the best-estimate in the time-consistent setting. The parameters are as follows: initial cohort $N_{x}=1000$, initial asset price $S_{0}=100$, risk-free interest rate $r=4 \%$, volatility $\sigma_{S}=15 \%$, and Lee-Carter model parameters as $\kappa_{0}=-24.5637$, $\mu_{\kappa}=-0.8089$, and $\sigma_{\kappa}=1.473^{10}$.

Figure 5.1 presents the result of the market-consistent pricing for a cohort of age $x=50$, which consists of the best-estimate, EIOPA risk-margin, and time-consistent price over different maturities $T \in\{1,2, \ldots, 30\}$. The equity and mortality risks are assumed to be independent. The graph shows that all prices decrease when maturity increases, indicating that the effect of the discount rate $r=4 \%$ is stronger than the mortality for the age group $x=50$. Additionally, for short-term liabilities, the difference between these marketconsistent prices is negligible, whereas for the longer term (15 years and longer), the gap widens through maturity. In fact, both EIOPA risk-margin and time-consistent actuarial prices measure the unhedgeable uncertainty related to the projected mortality on top of the best-estimate value. This gap is the risk loading that becomes larger when the maturity increases-a stylized fact that a longer time horizon is more uncertain. Because the best-estimate value is time consistent, the gap between its value and the time-consistent price is the time-consistent risk margin of the unhedgeable actuarial risk (or risk loading). This is the part of the price that we could not hedge under the market-consistent valuation. Moreover, the time-consistent price dominates the EIOPA price, where the difference is the capital-on-capital effect. This effect increases for long-dated contracts and, accordingly, is important to consider. If we divide the best-estimate price by $S_{0}=100$, the result shows the expected survivors for the cohort of age $x=50$ under the Lee-Carter model, from 997.5 for $T=1$ to 678.5 for $T=30$.

\section{Effect of Dependence on the Market-consistent Price}

We also study the effect of a possible dependence between mortality/longevity and equity risks on the market-consistent contract price for the different maturities. We choose four different levels of the correlation between $\kappa_{t}$ and $S_{t}$ as $\rho=\{0,0.50,0.75,1\}$, where $r h o=0$ shows independence and $\rho=1$ shows perfect hedging for actuarial risk attributable to the dynamics of financial risk in the complete market. In a formal manner, the effect of the dependence between financial and actuarial risk is reflected as a co-variance factor $\pi_{x y}$ in Eqs. (4.2.12) and (4.2.16) with positive coefficients " $\rho b \sigma$ " when $\rho>0$. However, note that if $\rho<0$, then $\pi_{x y}$ is also negative, which makes the entire term $\rho b \sigma \pi_{x y}$ and the covariance effect on price always positive. In Figure 5.2, for each correlation level (in graphs $(a)-(d)$ ), we provide the values of the three different prices previously discussed for the

\footnotetext{
${ }^{10}$ These parameters are estimated on the basis of mortality data aggregated for "men and women" of the Netherlands during the calendar years 1960-2006 (47 years).
} 


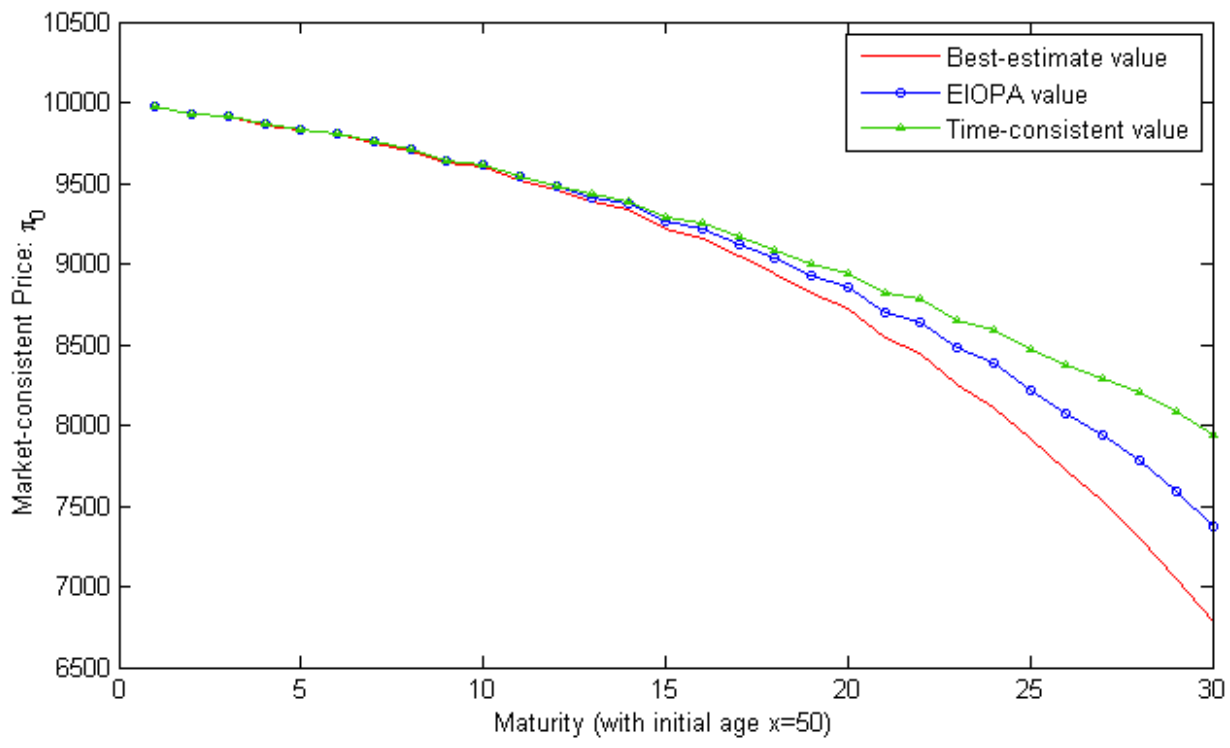

Figure 5.1: Comparison of the market-consistent actuarial valuation for the best-estimate, EIOPA risk-margin and time-consistent prices over different maturities $(T=1,2, \ldots, 30)$ for a cohort of age $x=50$. 

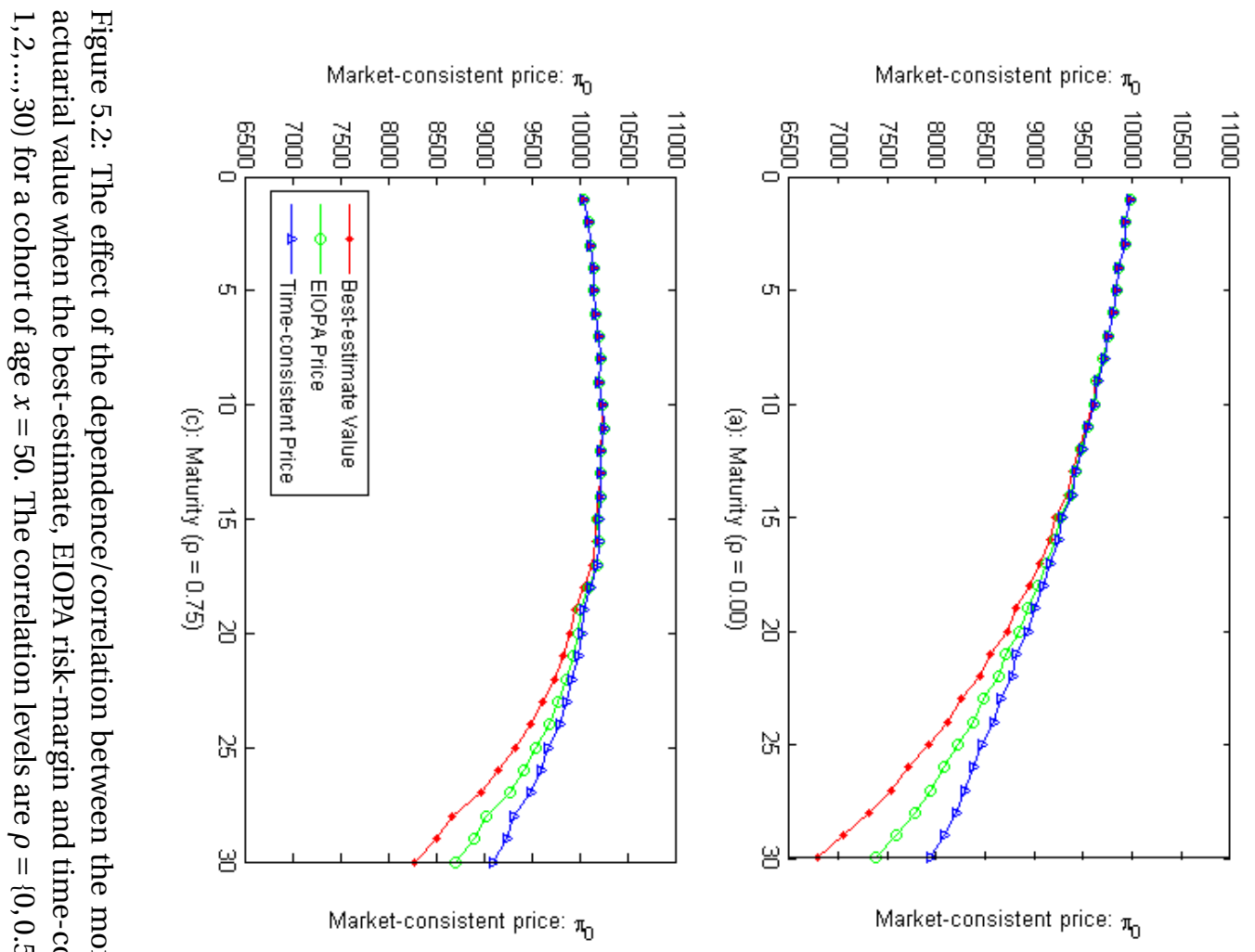

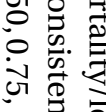
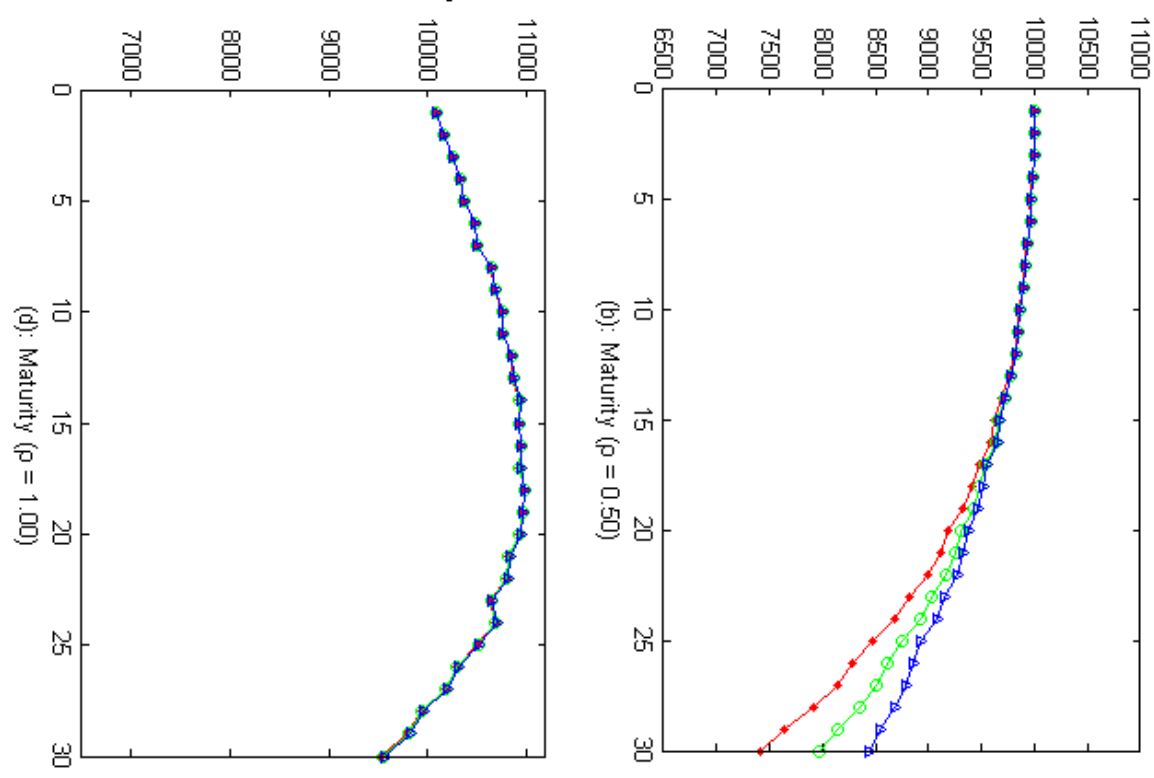

\&

ज 
cohort of age $x=50$ along different maturities.

In general, all prices illustrate that a larger correlation results in a higher marketconsistent price. This relationship reflects the extra price attributable to the co-variance factor in the price dynamics. Moving from the independent risks $(\rho=0)$ to the perfect hedge position $(\rho=1.00)$, the market-consistent prices for a 30-year unit-linked contract increase by $40.5 \%, 29.4 \%$, and $20.5 \%$, respectively, for the best-estimate, EIOPA, and timeconsistent values. Price growth is the result of three different effects. The first effect is an upward price movement from a change in the drift of financial risk in the $\mathbb{Q}$ measure. The second effect is the co-variance effect that, as discussed, increases the price. The third effect is the elimination of uncertainty as measured by the $99.5 \%$-quantile (risk-margin) that reduces the price. When the (absolute value of the) correlation increases, the drift adjustment and co-variance effect (the first two effects) fight against the uncertainty elimination effect (the third effect), and eventually win to increase the overall market-consistent actuarial price.

The results in Figure 5.2 are very important because they show that both EIOPA riskmargin and time-consistent prices approach the best-estimate when the correlation increases. This can be seen by following the sequence of the graphs from (a) to (d) in Figure 5.2 , and by observing the gap for the 30 -year maturity. Specially, in a perfect hedge situation, when $\rho=1$, both prices correspond exactly to the best-estimate price for all maturities (Graph $(\mathrm{d})$ ) because no unhedgeable risk remains. This adjusted best-estimate price has a different drift and dynamics relative to the original best-estimate price with $\rho=0$. Basically, we could show that for two different price operators (EIOPA and time-consistent prices), when financial and actuarial risks are perfectly correlated, the market-consistent price perfectly hedges actuarial risk on the basis of the information for financial risk in the complete market. This is good numerical evidence that the two-step actuarial operator in Definition 4.1.2 can guarantee market consistency for any price operator. 



\title{
6
}

Market-Consistent Valuation of the Participating Pension Contract

\author{
This chapter is based on: \\ Salahnejhad Ghalehjooghi, A. and Pelsser, A., (2016), Time-Consistent and Market- \\ Consistent Actuarial Valuation of the Participating Pension Contract, under review in \\ "ASTIN Bulletin".
}

Pension policies impose very long-dated liabilities that are not fully traded and hedgeable in the market. Due to existence of the actuarial risk in their payoff, they are normally priced by non-linear premium principles. Such long-term positions make the issuer's valuation and risk management vulnerable to the dynamics of meddle-time fluctuations. To reflect the effect of the meddle-time evolution on the price, we require time-consistency while the premium principles are not originally time-consistent. On the other hand, the regulator calls for market-consistent valuation for such liabilities, while they are not fully tradable. This study provides a time-consistent and market-consistent pricing method for a participating life/pension scheme with guaranteed interest rate. We consider a hybrid combination of the actuarial risk and hedgeable financial risks in the final payoff and the profit-sharing mechanism over the life of the contract. We achieve market-consistency by the "two-step actuarial valuation" proposed by Pelsser and Stadje (2014) and timeconsistency by repeating the one-period valuation procedure by a "backward iteration" in the sub-intervals over the valuation period. We use the Least-Square Monte-Carlo (LSMC) method to implement the backward iteration. We report the results by comparing the above price to the expected value of the discounted payoff and measure the relative risk loading and time-consistency risk premium. We also study the effect of the stochastic interest rate on the price of the contract as compared to the deterministic one. 


\subsection{Model and Setup}

The "participating policy" (i.e., with profit) is a practical, familiar form of pension contracts that has most of the main attributes of pension policies. These attributes include periodical crediting of a policy interest with a guaranteed rate, which is the link of the policy to the financial market.

We choose a well-structured version of the participating policy proposed by Grosen and Jorgensen (2000). According to the payoff and crediting mechanism, the policy is, by construction, linked to the Dutch pension payoff. In the Grosen-Jorgensen setting, at time $t=0$ a cohort of the policyholders buy one unit of the contract with nominal value $P_{0}$ for a single price $V_{0}$. The insurance company invests all its money in the financial market, and at the end of every year $t$ is committed to credit policy interest rate $r_{P}(t)$ that cannot be less than a guaranteed rate $r_{G}$. At the time of maturity $T$, the policy ends by paying a single value $P_{T}$ to each policyholder. We explain the liability dynamics and evolution of the policy reserve under the Grosen-Jorgensen setting in this section.

There is no actuarial risk involved in Grosen-Jorgensen's crediting mechanism. However, in the real world, apart from the financial factors, the longevity risk affects the evolution and maturity of the policy reserve on the liability sideof the balance sheet, through the number of survivors. To address this, we modify the Grosen-Jorgensen crediting mechanism to a hybrid financial and actuarial dynamic by incorporating the number of survivors $N_{x}(t)$ at time $t$ in formation of the policy reserve and the payoff, with reference to the starting cohort $N_{x}(0)$.

As the main focus of this study is implementing a market-consistent framework to price the participating pension contract, we skip the surrender option for the sake of simplicity. Later, in the numerical phase in Section 6.4, we calculate the price in two different situations where the risk-free interest rate is constant and stochastic. Thus, we analyze the effect of stochastic interest rate on the price of the contract.

\section{Risk Drivers}

The three main underlying risk drivers involved in the contract will be modeled as follows:

- Mortality/longevity (Lee-Carter): We model the mortality/longevity risk with the forecasting mortality Lee-Carter model. For an integer age $x$ in calendar year $t$, the Lee-Carter model assumes the following dynamics for the force-of-mortality $\mu_{x}(t)$

$$
\ln \mu_{x}(t)=\alpha_{x}+\beta_{x} \kappa_{t}
$$

that makes $\mu_{x}(t)$ a stochastic function with the underlying process $\kappa(t)$, which fol- 
lows a Brownian motion with drift as

$$
\mathrm{d} \kappa_{t}=\mu_{\kappa} \mathrm{d} t+\sigma_{\kappa} \mathrm{d} W_{t}^{\kappa}
$$

where $W_{t}^{\kappa}$ is the standard Brownian motion under the real world probability measure $\mathbb{P}$, and $\mu_{\kappa}$ and $\sigma_{\kappa}$ are constant. In the Lee-Carter model, $\kappa_{t}$ is the latent process to model the longevity trend over time, $\alpha_{x}$ is the average age-specific mortality, and $\beta_{x}$ is the age-specific sensitivity of the mortality to a change in $\kappa_{t}$.

Although applications of the model only use a discrete setting with a constant parameter over each year, the model can theoretically be applied to continuous-time dynamics.

- Investment Asset (Black-Scholes): We model the investment asset at time t, $A_{t}$, by a two-factor Geometric Brownian Motion (GBM)

$$
\mathrm{d} A_{t}=\mu_{A} A_{t} \mathrm{~d} t+\sigma_{A} A_{t} \mathrm{~d} \tilde{W}_{t}^{A} .
$$

where $\mu_{t}^{A}$ is the stochastic growth rate, $\sigma_{A}$ is the constant volatility, and $W_{t}^{A}$ is a standard Brownian motion under the real-world measure $\mathbb{P}$. As we assume that the financial market is complete and arbitrage-free, there exists a unique equivalent martingale measure $\mathbb{Q}$ according to the second fundamental theorem of asset pricing, for which $A_{t}$ follows the risk-adjusted GBM below

$$
\mathrm{d} A_{t}=r_{t} A_{t} \mathrm{~d} t+\sigma_{A} A_{t} \mathrm{~d} W_{t}^{A} .
$$

with the solution

$$
A_{t}=A_{0} \exp \left(\left(r_{t}-\frac{1}{2} \sigma_{A}^{2}\right) t+\sigma_{A} W_{t}^{A}\right),
$$

where $A_{0}$ is the initial value of the investment, $W_{t}^{A}$ is standard Brownian motion under $\mathbb{Q}$, and $r_{t}$ is the continuously compounded stochastic interest rate (also called short rate).

- Interest rate (Hull-White): We use the one-factor Hull-white short rate model for the term structure of the stochastic interest rate described by the following stochastic differential equation

$$
\mathrm{d} r_{t}=\left[\theta(t)-a r_{t}\right] \mathrm{d} t+\sigma_{r} \mathrm{~d} W_{t}^{r}
$$

where $\theta(t)$ is the time varying parameter chosen in such a way that the model fits the initial term structure in the market. $a$ and $\sigma_{r}$ are positive constants, and $W_{t}^{r}$ is the standard Brownian motion under the risk-neutral measure $\mathbb{Q}$. 
The three underlying risk drivers have the following correlation matrix

$$
\vec{\rho}=\left(\begin{array}{ccc}
1 & \rho_{\kappa, r} & \rho_{\kappa, A} \\
\rho_{\kappa, r} & 1 & \rho_{A, r} \\
\rho_{\kappa, A} & \rho_{A, r} & 1
\end{array}\right)
$$

where all $\left|\rho_{A, r}\right|<1$. If we assume the longevity risk is independent from $A_{t}$ and $r_{t}$, we set $\rho_{\kappa, A}$ and $\rho_{\kappa, r}$ equal to zero.

\section{Liability Dynamics and Payoff}

Consider pricing a policy with maturity $T$ at time $t=0$. Let $r_{P}(t)$ be the policy interest rate, $A_{t}$ be the "market value of the assets," and $P_{t}$ be the "policy reserve." The difference $B_{t}=A_{t}-P_{t}$ is called the "bonus reserve" ${ }^{1}$. The financial value of the policy reserve is then,

$$
P_{t}=P_{t-1} \times\left(1+r_{P}(t)\right) \quad, \quad t=1,2, \ldots, T
$$

which is equivalent to $P_{0}$ as $P_{t}=P_{0} \prod_{i=1}^{t}\left(1+r_{P}(i)\right)$.

\section{Grosen-Jorgensen Mechanism}

Under the Grosen-Jorgensen setting, $P_{t}$ is a pure financial driver and the crediting mechanism of the policy interest rate at each year $t$ is a function of $A_{t}, r_{G}$, and the ratio of the management decision on the buffer, $B_{t} / P_{t}$. If the management determines a "target buffer ratio" $\gamma$, and if $B_{t} / P_{t}>\gamma$, the fund will distribute a positive fraction $\alpha$ of the excessive amount of the buffer ratio above $r_{G}$ in form of the policy interest rate. ${ }^{2}$ Since, in any year $t$ (i.e., the time interval $(t-1, t)$ ), the fund management typically decides $r_{P}(t)$ based on the state of the fund in the previous year (i.e., $P_{t-1}$ and $B_{t-1}$ ), the analytical formula for $r_{P}(t)$ is ${ }^{3}$

$$
r_{P}(t)=\max \left\{r_{G}, \alpha\left(\frac{B_{t-1}}{P_{t-1}}-\gamma\right)\right\} .
$$

Thus, the equation (6.1.8) can be updated as

$$
\begin{aligned}
P_{t} & =P_{t-1}\left(1+\max \left\{r_{G}, \alpha\left(\frac{B_{t-1}}{P_{t-1}}-\gamma\right)\right\}\right) \\
& =P_{t-1}\left(1+r_{G}+\max \left\{0, \alpha\left(\frac{A_{t-1}-P_{t-1}}{P_{t-1}}-\gamma\right)-r_{G}\right\}\right)
\end{aligned}
$$

\footnotetext{
${ }^{1} P_{t}+B_{t}$ constitute the liability side of the balance sheet at time $t$.

${ }^{2} \alpha$ is called the "distribution ratio," the realistic value of which is $20-30 \%$. Additionally, the usual value of $\gamma$ is around $10-15 \%$ (Grosen and Jorgensen (2000)).

${ }^{3}$ By crediting $r_{P}(t)$ at year $t$, we mean that the interest rate is credited for the period of $(t-1, t)$, and hence is determined at time $t-1$.
} 
In this mechanism, the bonus interest rate is an option element with the strike value $r_{G}$, whereas $P_{t}$ is a path-dependent process with respect to $A_{t}$; as a result, we cannot find an analytical solution for the value. We can also rewrite the Eq. (6.1.10) as

$$
P_{t}=P_{t-1}\left(1+\max \left\{r_{G}, \alpha\left(\frac{A_{t-1}}{P_{t-1}}-(1+\gamma)\right)\right\}\right)
$$

where the index $A_{t-1} / P_{t-1}$ is the well-known "funding ratio" of the pension fund, and accordingly $1+\gamma$ is the "target funding ratio". 4 The above formulation is in fact a dynamic "profit-sharing" mechanism that allocates potential extra profit based on the ratio of the assets and liabilities every year. Note that, this profit-sharing mechanism is driven only by the financial risks and not by actuarial risk.

\section{Hybrid Payoff and Funding Mechanism}

To capture the fact that the liability side also depends on the actuarial risk, we add the mortality/longevity factor to the Grosen-Jorgensen setting.

We start with forming the hybrid payoff. Let $N_{x}(t)$ denote the cohort of the policyholders of age $x+t$ at time $t$, with the initial cohort $N_{x}(0)$ of the policyholders who bought the policy at time $t=0$. Each policyholder receives benefits of $P_{T}^{(h)}$ only if he/she is alive at maturity $T$ (i.e., at age $x+T$ ), and zero otherwise. This arises from the implicit mechanism of insurance in the pension policy where a cohort pays the premium, but less people receive the benefits ${ }^{5} . P_{T}^{(h)}$ is the hybrid version of the policy reserve, $P_{T}$, made by the hybrid crediting mechanism that will be explained a bit later in this section. The discounted general payoff at time $T$ is then

$$
G\left(P_{T}, r_{T}, \kappa_{T}\right)=\left(e^{-\int_{0}^{T} r_{t} d t}\right) \times P_{T}^{(h)} \times N_{x}(T) .
$$

This general payoff is a function of the underlying risk drivers $A_{T}, P_{T}$, and $r_{T}$ for financial risks, and $\kappa_{T}$ for longevity risk in the Lee-Carter model, as described in subsection 6.1. However, note that at maturity $T$, the value of $P_{T}$ will be known by $A_{T-1}$ and $P_{T-1}$. Thus, we omitted $A_{T}$ in the payoff function and used only $P_{T}$. Moreover, the payoff represents a European style contract without the surrender option before maturity.

Apart from the final payoff, we are also interested to know how mortality/longevity risk is involved in the funding and crediting mechanism. We look at this problem from the funding management point of view. In the Grosen-Jorgensen setting, where the crediting

\footnotetext{
${ }^{4}$ This crediting mechanism and the fund evolution is also called the "Indexation Ladder" in the literature.

${ }^{5}$ In most countries, this is a realistic case where those who do not survive until retirement age (or their beneficiaries) cannot benefit from the money they deposit to the fund. The money goes towards compensating the retirement salary of those who live longer.
} 
mechanism is purely made of the financial dynamics, in each period of time $(t-1, t)$, if the funding ratio at the start of the period exceeds the target funding ratio $1+\gamma$, the managers will consider paying $\alpha$ part of the extra benefit to the remaining policyholders if the resulting interest is higher than the guaranteed interest rate, $r_{G}$. Additionally, at maturity $T$, the total value of the policy reserve is $N_{x}(T) \times P_{T}$. Therefore, path-dependency is only due to the asset value and interest rate and not based on the number of survivors over the life of the contract. Pricing under the Grosen-Jorgensen mechanism implicitly postulates that the fund managers only take into account the time-zero expectation of the survivors at maturity $T$ and do not change this perception based on the realization of $N_{x}(t)$ over the contract term.

The estimation or perception of the number of survivors at maturity, $N_{x}(T)$, depends on the realization of the time- $t$ number of survivors, $N_{x}(t)$ over the contract period (which itself is based on the state of the longevity trend $\kappa_{t}$ ). We measure this time- $t$ perception by the best-estimate of $N_{x}(T)$ at time $t$, denoted by $B E_{t}\left(N_{x}(T)\right)$, which is defined as its conditional expectation given the information available at time $t$,

$$
B E_{t}\left(N_{x}(T)\right)=\mathbb{E}\left[N_{x}(T) \mid N_{x}(t)\right]
$$

In fact, going forward over the valuation period to time $t$, the best-estimate value of $N_{x}(T)$ can be updated with the new information of the underlying process $\kappa_{t}$ and $N_{x}(t)$.

Suppose that the fund manager wants to calculate the policy interest rate at time $t$. Apart from the financial dynamics in the Grosen-Jorgensen setting, if at time $t$, $B E_{t}\left(N_{x}(T)\right)$ is higher than $B E_{0}\left(N_{x}(T)\right)$ (the initial perception of the fund manager on the terminal number of survivors), the situation will be more risky. In this situation, the fund manager will expect that the final collective policy reserve for the whole pension fund has to be divided among more survivors at time $T$, which means that the individual policy reserve at maturity, $P_{T}$ has to be less than the Grosen-Jorgensen policy reserve. This can be seen from the payoff formula in Eq. (6.1.12) where, if $N_{x}(T)$ increases, $P_{T}$ has to decrease so that the overall liability remains the same. This may convince the fund managers to promise a lower policy interest rate $r_{P}$. In order to do so, they may pursue a more conservative policy for profit-sharing either by setting a higher target funding ratio $(1+\gamma)$ or reducing the distribution ratio $(\alpha)$. This will automatically result in a lower bonus interest rate in excess of $r_{G}$. On the other hand, if $B E_{t}\left(N_{x}(T)\right)<B E_{0}\left(N_{x}(T)\right)$, the updated estimation of the final survivors at maturity may convince the fund manager to set a more liberal profit-sharing policy by changing $1+\gamma$ or $\alpha$. The reason is that he will expect less people at maturity to get the policy reserve $P_{T}$; this is translated into a higher policy reserve with a higher promised interest rate.

Using these facts, we can construct a hybrid crediting mechanism for the participating policy where the estimation of the final number of survivors affects crediting and profit 
sharing over the life of the contract. Under the hybrid mechanism, we assume that each survivor of the terminal cohort (at maturity $T$ ), $N_{x}(T)$, has an equal share of the fund's total assets and policy reserve. We define the hybrid funding ratio at time $t$ as

$$
\text { Hybrid funding ratio }=\frac{B E_{0}\left(N_{x}(T)\right) A_{t}}{B E_{t}\left(N_{x}(T)\right) P_{t}}=\frac{\mathbb{E}\left[N_{x}(T) \mid N_{x}(0)\right]}{\mathbb{E}\left[N_{x}(T) \mid N_{x}(t)\right]} \times \frac{A_{t}}{P_{t}} .
$$

where at each point of time $t$, the Grosen-Jorgensen funding ratio, $A_{t} / P_{t}$, is modified by the ratio of the time-zero best-estimate of the final survivors to its time- $t$ best-estimate.

By this hybrid funding ratio, we implicitly focus on the final survivors $N_{x}(T)$, against whom the fund bears liability. During the contract period at any time $t$, the final survivors own the "best-estimate" value of $N_{x}(T)$ multiplied by the value of the asset $A_{t}$ or policy reserve $P_{t}$. The initial asset value and policy reserve for the whole cohort is then $B E_{0}\left(N_{x}(T)\right) \times A_{0}$ and $B E_{0}\left(N_{x}(T)\right) \times P_{0}$, respectively. However, as the investment asset evolves only through the dynamics of the financial market, and not through the mortality/longevity trend, we only update the best-estimate of the final cohort for the policy reserve; and we maintain our best estimate at time $t=0$ for the asset side.

Let us denote all the quantities related to the hybrid mechanism with a superscript “(h)”. The modified formulation for hybrid policy interest rate is

$$
r_{P}^{(h)}(t)=\max \left\{r_{G}, \alpha\left(\frac{B E_{0}\left(N_{x}(T)\right)}{B E_{t-1}\left(N_{x}(T)\right)} \times \frac{A_{t-1}}{P_{t-1}}-(1+\gamma)\right)\right\} .
$$

The hybrid funding ratio introduces a hybrid profit-sharing mechanism (year-on-year) dependent on two main components: the "investment component" and the "technical component". The investment component is a pure financial quantity that corresponds to the same funding ratio in the Grosen-Jorgensen setting, and the technical component is an actuarial ratio of the best-estimate number of survivors mentioned above as $B E_{0}\left(N_{x}(T)\right) / B E_{t-1}\left(N_{x}(T)\right)$. Hence, both investment and technical components are dynamic processes and make the payoff a path-dependent process with respect to both investment assets and longevity trend through the above mechanism.

Figure 6.1 illustrates five simulated paths of the market value of the invested assets $A_{t}$ and the hybrid policy reserve for a 20 -year pension contract with a guaranteed interest rate $r_{G}=2 \%$. Clearly, comparing to Grosen-Jorgensen mechanism, the above hybrid mechanism smooths the policy interest rate more in the sense of having lower volatility.

In Eq. (6.1.15), we can rewrite the target funding ratio $1+\gamma$ as a product of the technical component and a new "hybrid target buffer ratio", $1+\gamma_{t-1}^{(h)}$,

$$
1+\gamma=\frac{B E_{0}\left(N_{x}(T)\right)}{B E_{t-1}\left(N_{x}(T)\right)} \times 1+\gamma_{t-1}^{(h)}
$$

This shows that even if $\gamma$ in the managerial protocol may be determined as a constant 


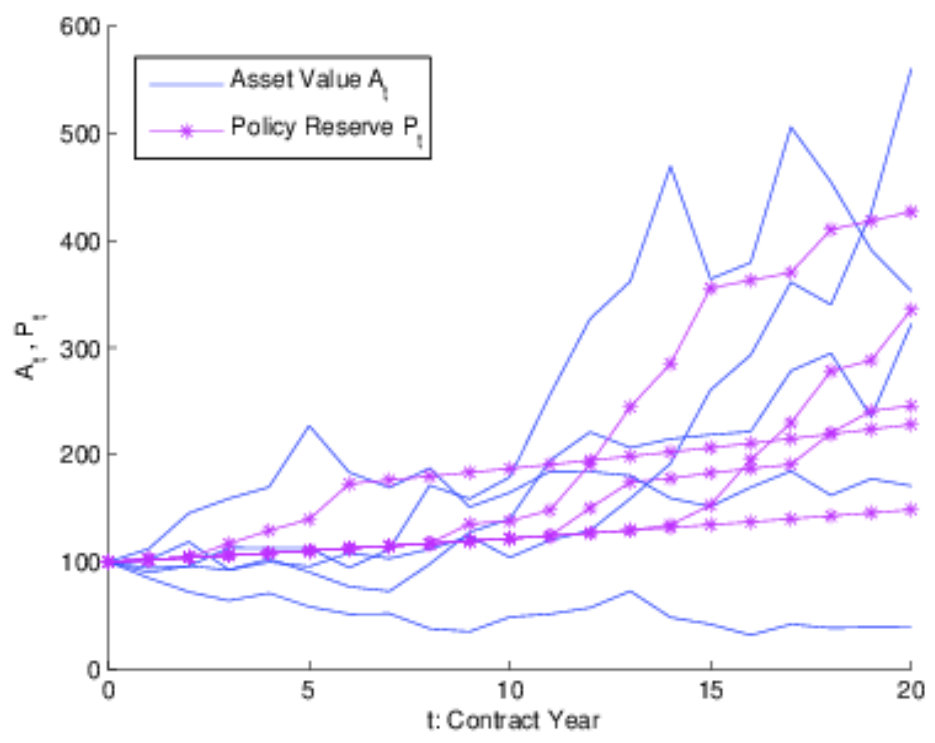

Figure 6.1: Simulation of the Asset Value $A_{t}$ and Policy Reserve $P_{t}$ of the Pension Contract. Parameter set: $S_{0}=P_{0}=100, r=4 \%, \sigma_{S}=0.15, r_{G}=2 \%$.

value, technically at any time $t$ it can be transformed into a dynamic process $\gamma_{t-1}^{(h)}$ under the hybrid crediting mechanism. Eq. (6.1.15) can be rewritten as follows

$$
r_{P}^{(h)}(t)=\max \left\{r_{G}, \alpha \times \frac{B E_{0}\left(N_{x}(T)\right)}{B E_{t-1}\left(N_{x}(T)\right)}\left(\frac{A_{t-1}}{P_{t-1}}-\left(1+\gamma_{t+1}^{(h)}\right)\right)\right\} .
$$

where one can also consider $\alpha \times\left(B E_{0}\left(N_{x}(T)\right) / B E_{t-1}\left(N_{x}(T)\right)\right)$ as a new "dynamic distribution ratio" under the hybrid profit-sharing mechanism.

Finally, the recursive formulas for the policy reserve in Eq. (6.1.11) is modified for the hybrid policy reserve as

$$
\begin{aligned}
P_{t}^{(h)} & =P_{t-1}^{(h)} \times\left(1+r_{P}^{(h)}(t)\right) \\
& =P_{t-1}^{(h)} \times\left(1+\max \left\{r_{G}, \alpha\left(\frac{A_{t-1}}{P_{t-1}^{(h)}}-\left(1+\gamma_{t-1}^{(h)}\right)\right)\right\}\right) .
\end{aligned}
$$

where at time $t-1$ by considering Eq. (6.1.17), the hybrid policy reserve, $P_{t-1}^{(h)}$ is obtained from the financial policy reserve as follows

$$
P_{t-1}^{(h)}=\left(\frac{B E_{t-1}\left(N_{x}(T)\right)}{B E_{0}\left(N_{x}(T)\right)}\right) \times P_{t-1}
$$


Example 6.1.1. Suppose $N_{x}(t)$ follows a geometric Brownian motion with a constant drift $a<0$ and volatility. Hence, it will be log-normally distributed with mean $N_{x}(0) \exp (a T)$. Accordingly, the technical component (i.e., the ratio of the best-estimate of the terminal cohort) in Eq. (6.1.14) can be rewritten as

$$
\begin{aligned}
\frac{\mathbb{E}\left[N_{x}(T) \mid N_{x}(0)\right]}{\mathbb{E}\left[N_{x}(T) \mid N_{x}(t)\right]} & =\frac{N_{x}(0) \times e^{a(T)}}{N_{x}(t) \times e^{a(T-t)}} \\
& =\frac{N_{x}(0) \times e^{a(t)}}{N_{x}(t)} .
\end{aligned}
$$

The log-normal cohort exhibits clear interpretation of the technical component, which is the ratio of the expected number of survivors to the realized number of survivors at time $t$.

For the log-normal cohort, using Eq. (6.1.19), $r_{P}^{(h)}(t)$ can be rearranged as

$$
r_{P}^{(h)}(t)=\max \left\{r_{G}, \alpha \frac{N_{x}(0) \times e^{a(t-1)}}{N_{x}(t-1)}\left(\frac{A_{t-1}}{P_{t-1}}-(1+\gamma)\right)\right\}
$$

where we implicitly assumed that $1+\gamma^{(h)}=\left(N_{x}(0) \times e^{a(t-1)} / N_{x}(t)\right)(1+\gamma)$.

The hybrid crediting mechanism adds some extra insights to the Grosen-Jorgensen setting. Suppose in year $t$, the realized number of survivors is larger than the expected number of survivors; $N_{x}(t)>N_{x}(0) \times e^{a(t-1)}$. This deviation can be interpreted as a "local realization of the longevity risk." Then, the technical component will act on the investment component of the funding ratio as a diminishing factor that, on average, decreases the policy interest rate closer to $r_{G}$. This means that when there is a sign of the longevity risk in the portfolio, the hybrid mechanism in Eq. (6.1.21) will impose a "conservative crediting" on the policy interest rate. On the other hand, if the realized number of survivors is less than the expected number of them, $N_{x}(t)<N_{x}(0) \times e^{a(t-1)}$, the technical component is larger than 1 , and will act as an enlarging factor of the investment component, result in a higher policy interest rate. In this situation, in comparison to the Grosen-Jorgensen mechanism, the hybrid setting creates more liberal reactions of the fund to interest crediting.

\subsection{Pricing Framework}

The payoff in Eq. (6.1.12) is an example of the wide-variety of derivatives that are a combined function of the underlying financial and actuarial risks. We consider the mortality/longevity (with underlying risk process $\kappa_{t}$ ) as an unhedgeable (or partially hedgeable) actuarial risk, where the no-arbitrage argument cannot be applied due to market incompleteness. Instead, we use one of the actuarial premium principles to price it. The premium principles are normally non-linear operators that do not possess the marketconsistency and time-consistency properties, and normally operate in $\mathrm{n}$ static way. On 
the other hand, the interest rate $\left(r_{t}\right)$ and investment asset $\left(A_{t}\right)$ (and accordingly the policy reserve $P_{t}$ ) are the financial risks that are assumed to be fully hedgeable and traded in an arbitrage-free and complete market. Hence, with the fundamental theorem of asset pricing, the price of the derivative made of $A_{t}$ and $r_{t}$, is a conditional expectation under the unique risk-neutral martingale measure $\mathbb{Q}$.

We would like to price the participating policy simultaneously under the marketconsistency and time-consistency properties.

\section{Two-step Actuarial Valuation}

In Chapters 4 and 5, we used the result of the paper by Pelsser and Stadje (2014) that shows any market-consistent operator can be constructed by a "two-step valuation" that splits the actuarial and no-arbitrage financial pricing operators for every valuation period. For a pricing operator that is time-consistent over only a finite number of points in the valuation period, the two-step valuation preserves the market-consistency on the same points. Using this property, we mixed the two-step actuarial valuation and backward iteration to introduce an applied representation for time-consistent and market-consistent price in discrete-time and continuous-time.

Let $(\Omega, \mathscr{F}, \mathbb{P})$ be the underlying probability space with the filtration $\left(\mathscr{F}_{t}\right)_{t \geq 0}$ over $[0, T]$. Suppose $\left(\mathscr{F}_{t}^{A}\right)_{t \geq 0},\left(\mathscr{F}_{t}^{r}\right)_{t \geq 0}$ and $\left(\mathscr{G}_{t}^{A}\right)_{t \geq 0}$ denote the filtrations generated by the policy reserve $P_{t}$, interest rate $r_{t}$, and mortality trend $\kappa_{t}$, which all are subsets of $\mathscr{F}$. We form the set $\mathscr{F}_{t}^{S}=\sigma\left(\mathscr{F}_{t}^{A} \cup \mathscr{F}_{t}^{r}\right)$ as the filtration for the combination of financial risk drivers $P_{t}$ and $r_{t}$ and $\mathscr{G}_{t}=\sigma\left(\mathscr{F}_{t}^{S} \cup \mathscr{G}_{t}^{A}\right)$, as the hybrid filtration generated by the hybrid payoff which provides the information flow of the both financial and actuarial risks together at time $t$.

\section{Two-step Operator}

Suppose we are at time $t$ and let $\pi_{t}$ be the price of the payoff in Eq. (6.1.12) conditional on the information available at time $t$. Salahnejhad and Pelsser (2016) consider the operator for only two risk drivers (one financial and the other actuarial). We extend the same framework for three risk drivers (two financial and one actuarial) where, the market-consistent price of the participating policy at time $t<T$ is obtained by the following two-step operator

$$
\pi_{t}\left(A_{T}, r_{T}, \kappa_{T}\right)=\mathbb{E}^{\mathbb{Q}}\left[\Pi^{\mathbb{P}}\left[\left(e^{-\int_{t}^{T} r_{s} d s}\right) \times P_{T}^{(h)} \times N_{x}(T) \mid \sigma\left(\mathscr{G}_{t}^{A} \cup \mathscr{F}_{T}^{S}\right)\right] \mid \sigma\left(\mathscr{G}_{t}^{A} \cup \mathscr{F}_{t}^{S}\right)\right]
$$

where $\Pi$ can act as one of the actuarial premium principles. It is easy to examine that the above two-step operator is market-consist.

The above two-step operator is a one-period valuation consist of an "Inner Step" and an "Outer Step". In the inner step, we assume that we know the full financial information 
related to the policy reserve and term structure of the interest rate up to and including maturity $T$, but our information regarding the mortality trend $\kappa$ is limited to time $t<T$. Hence, the only randomness comes from $\kappa_{T}$. Here, we use slightly different version of hybrid $\sigma$-algebra $\mathscr{G}$ where there is time difference between the financial filtration up to $T$ and the actuarial filtration up to $t<T$. We can show the hybrid filtration as $\mathscr{G}_{T, t}=\sigma\left(\mathscr{F}_{T}^{S} \cup \mathscr{G}_{t}^{A}\right)$. In the following, by fixing $A_{T}$ and $r_{T}$, we use $\Pi^{\mathbb{P}}$ as the actuarial premium principle under the real world measure $\mathbb{P}$ and calculate the actuarial part of the payoff conditional on $\mathscr{G}_{T, t}$. The result of the inner step will then be a function of only policy reserve $P_{T}$ and the short rate $r_{t}$ from $t$ up to $T$. We show the value by $G^{S}\left(t, T, P_{T}, r_{T}\right)$. As we assumed, $G^{S}$ is a fully hedgeable position tradable in the no-arbitrage and complete market. Hence, in the outer step, we use the no-arbitrage pricing operator and take the expectation conditional on the available operators on their financial information $\mathscr{F}_{t}^{S}$ under the unique risk-neutral measure $\mathbb{Q}$.

We assume that $A_{t}, r_{t}$, and $\kappa_{t}$ have Markov property and are adapted to the corresponding information flows $\mathscr{F}_{t}^{A}, \mathscr{F}_{t}^{r}$, and $\mathscr{G}_{t}^{A}$. Thus, for simplicity we will use the value of the processes instead of the related filtrations in the pricing operators. Nevertheless, the alternative notation (with use of filtrations) is also valid for all the two-step conditional operators.

Now, we also want to include the time-consistency to the two-step valuation in Eq. (6.2.1). Let us choose Standard-Deviation premium principle as the actuarial price operator $\Pi$ in the inner step. By recalling Eq. (6.2.1), the one-period Standard-Deviation actuarial price will be defined as,

$$
\begin{aligned}
\pi_{t}\left(A_{T}, r_{T}, \kappa_{T}\right)=\mathbb{E}^{\mathbb{Q}}\left[\mathbb{E}^{\mathbb{P}}\left[\left(e^{-\int_{T-1}^{T} r_{s} \mathrm{~d} s}\right) \times P_{T}^{(h)} \times N_{x}(T) \mid \kappa_{t}, A_{T}, r_{T}\right]\right. \\
\left.+\beta \sqrt{T} \sqrt{\operatorname{Var}^{\mathbb{P}}\left[\left(e^{-\int_{T-1}^{T} r_{s} \mathrm{~d} s}\right) \times P_{T}^{(h)} \times N_{x}(T) \mid \kappa_{t}, A_{T}, r_{T}\right]} \mid \kappa_{t}, A_{t}, r_{t}\right]
\end{aligned}
$$

where the coefficient $\sqrt{T}$ is multiplied to $\beta$ to match the dimensionality problem of the square root function. For more details, please see the related discussion in our paper (Pelsser and Salahnejhad, 2016) or Chapter 2 in this thesis.

We assume that the price is time-consistent on a finite predictable points of time $t \in$ $\{0,1,2, \ldots, T-1, T\}$. Hence, in a multi-period setting, we apply the backward iteration of the two-step actuarial valuation and we repeat the valuation on an annual basis ${ }^{6}$ starting from $[T-1, T]$ and ending up at $[0,1]$. Hence, the time-consistent and market-consistent

\footnotetext{
${ }^{6}$ As the estimations in Lee-carter model are normally piece-wise annually constant, we focus on annual partitioning over the valuation process. However, the representation is valid for a general case where $[0, T]$ is divided into a partitioning of a shorter or longer sub-intervals with length $\Delta t$ as $\{0, \Delta t, 2 \Delta t, \ldots ., T-\Delta t, T\}$.
} 
Standard-Deviation pricing operator for the participating policy payoff in Eq. (6.1.12) is

$$
\begin{aligned}
\pi_{t}\left(A_{t+1}, r_{t+1}, \kappa_{t+1}\right) & =\mathbb{E}^{\mathbb{Q}}\left[\mathbb{E}^{\mathbb{P}}\left[\left(e^{-\int_{t}^{t+1} r_{s} \mathrm{~d} s}\right) \pi_{t+1}\left(A_{t+1}, r_{t+1}, \kappa_{t+1}\right) \mid \kappa_{t}, A_{t+1}, r_{t+1}\right]\right. \\
+ & \left.\beta \sqrt{\operatorname{Var}^{\mathbb{P}}\left[\left(e^{-\int_{t}^{t+1} r_{s} d s}\right) \pi_{t+1}\left(A_{t+1}, r_{t+1}, \kappa_{t+1}\right) \mid \kappa_{t}, A_{t+1}, r_{t+1}\right]} \mid \kappa_{t}, A_{t}, r_{t}\right]
\end{aligned}
$$

with terminal condition

$$
\pi_{T}\left(A_{T}, r_{T}, \kappa_{T}\right)=\left(e^{-\int_{T-1}^{T} r_{s} \mathrm{~d} s}\right) \times P_{T}^{(h)} \times N_{x}(T)
$$

where $\beta>0$ is the loading coefficient. The operator is constructed under time-consistency argument, where the payoff applicable in each time-step of the form $(t, t+1)$ is the one-period two-step value obtained one time-step further in $(t+1, t+2)$. The integral $\left(\int_{t}^{t+1} r_{s} \mathrm{~d} s\right)$ represents the $t$-forward interest rate accumulated over the time-step $(t, t+1)$.

\section{(In)Dependence Structure}

Due to use of the conditional operators, the two-step valuation is by construction consistent with existence of a dependence structure between the financial and actuarial risk drivers. If there is such dependence, the actuarial risk will be partially hedged via the financial risk. This means that in Eq. (6.2.1) by knowing the full financial information $\mathscr{F}_{T}^{S}$, we know part of the actuarial information $\mathscr{G}_{T}^{A}$. Therefore, if in the inner step we fix the Markov process $P_{T}$ and $r_{T}$, we have some information to forecast the value of $\kappa_{T}$. As we assume $P_{T}$ and $r_{T}$ are fully hedgeable, a part of the position with underlying $\kappa_{T}$ can be hedged in the outer step by the arbitrage-free operator $\mathbb{E}^{\mathrm{Q}}$ due to the common information available in the financial market. We discussed this for the continuous-time limit of the time-consistent two-step Variance and Standard-Deviation operators in Chapter 2. We showed that the underlying actuarial process will be adjusted by a function of the correlation that plays the role of the hedge cost, to partially hedge the actuarial process in the market. The continuous-time time-consistent and market-consistent price is represented in their study by a partial differential equation which does not have an explicit analytical solution and must be solved numerically.

However, in reality it is difficult to find a justification that the level and shape of the term structure and/or value of the investment asset can affect the mortality trend or vice versa. In Chapter 5, we considered a unit-linked payoff with a similar structure to the Grosen-Jorgensen case in Eq. (6.1.11), where the final payoff $P_{T} \times N_{x}(T)$, is built based on a pure financial procedure. Due to the factorized structure of the payoff, for an independent policy reserve and mortality risks, the inner and outer operators in the two-step valuation can be split into the product of the operators as below

$$
\pi_{t}\left(P_{T}, \kappa_{T}\right)=e^{-r(T-t)} \mathbb{E}^{\mathbb{Q}}\left[P_{T} \mid \mathscr{F}_{t}^{S}\right] \times \Pi^{\mathbb{P}}\left[N_{x}(T) \mid \mathscr{G}_{t}^{A}\right]
$$


where there is no shared information generated by the two risk drivers. ${ }^{7}$ Earlier in Chapter 3 , we showed that in a continuous-time mortality model, applying time-consistency for the actuarial operator $\Pi^{\mathbb{P}}$ and considering the fact that the expectation operator $\mathbb{E}$ by construction is time-consistent, the two-step Standard-Deviation operator for the GrosenJorgensen payoff will be obtained as

$$
\pi_{t}\left(P_{T}, \kappa_{T}\right)=e^{-r(T-t)} \mathbb{E}^{\mathbb{Q}}\left[P_{T} \mid P_{t}\right] \times \mathbb{E}^{\mathbb{S}}\left[N_{x}(T) \mid \kappa_{t}^{\mathbb{S}}\right]
$$

where $\mathbb{E}^{\mathbb{S}}\left[. \mid \kappa_{t}^{\mathbb{C}}\right]$ is the conditional expectation with respect to the information available at time $t$ under the underlying risk-adjusted process

$$
\mathrm{d} \kappa_{t}^{\mathbb{S}}=\left(\mu_{\kappa}-\beta \sigma_{\kappa}\right) \mathrm{d} t+\sigma_{\kappa} \mathrm{d} W_{t}^{\mathbb{S}, \kappa} .
$$

For the hybrid mechanism, we set the independence assumption between the actuarial risk $\kappa_{t}$ and the financial risks $A_{t}$ and $r_{t}$, while $A_{t}$ and $r_{t}$ are correlated. Despite the independence assumption, the payoff in Eq. (6.1.12) cannot be factorized to a product of the financial and actuarial risks, as $P_{T}^{(h)}$ is a hybrid path-dependent function of $A_{t}$ and $\kappa_{t}$. Hence, giving an explicit solution for the time-consistent two-step Standard-Deviation price is not possible, and we have to calculate the price numerically.

\subsection{Numerical Method}

In this section, we explain the implementation of the numerical methods to calculate the time-consistent and market-consistent actuarial price of the participating contract. The numerical method is a combination of Monte-Carlo simulation and the regressionbased methods to calculate the values in the two-step actuarial operator and necessary conditional expectations. We focus on generating the scenarios of the payoff in Eq. (6.1.12) via simulation of the underlying risk drivers investment asset value $A_{t}$, interest rate $r_{t}$, and the longevity trend $\kappa_{t}$ when $t \in\{0,1, \ldots, T\}$. To do so, we calculate the hybrid policy interest rate $r_{P}^{(h)}(t)$, and the policy reserve $P_{t}^{(h)}$ via Eqs. (6.1.15) and (6.1.18), respectively. To achieve time-consistency, we use the backward iteration method to repeat the oneperiod two-step actuarial value over the valuation period with regression-based methods.

\footnotetext{
${ }^{7}$ According to Pelsser and Stadje (2014), this is valid for all payoffs with factorized form of the financial and actuarial derivatives.
} 
Simulation of $r_{P}^{(h)}$ and $P_{t}^{(h)}$

Using the correlation matrix in Eq. (6.1.7) and its Cholesky decomposition, we simulate a three-dimensional Brownian motion $\left(W_{t}^{\kappa}, W_{t}^{A}, W_{t}^{r}\right)^{\top}$ for $t \in 0,1, \ldots, T{ }^{8}$ Then, we use the dynamics in Section 6.1 to construct a sequence of the investment asset $A_{t}$, interest rate $r_{t}$, and longevity trend $\kappa_{t}$. For the interest rate $r_{t}$, we first generate the following OrnsteinUhlenbeck process

$$
d X_{t}=-a X_{t} \mathrm{~d} t+\sigma \mathrm{d} W_{t}^{r}
$$

where $X_{0}=0$ and $r_{t}=X_{t}+\alpha(t) . \alpha(t)$ can be determined by fitting $X_{t}$ to an initial term structure from the bond market and finally get the tractability parameter $\theta(t)$ in Eq. (6.1.6). See Pelsser (2000) for more details on transformation of $r_{t}$ and $X_{t}$ and obtaining the tractability parameter $\theta(t)$. The simulation will be performed for all three risks with replication $n$, each useful for one path, and the final output will be three $n \times(T+1)$ matrix for $A_{t}, r_{t}$ and $\kappa_{t}$.

To simulate the payoff in Eq. (6.1.12) we should generate $N_{x}(t)$ and $r_{P}^{(h)}(t)$. Recalling the Lee-Carter dynamic for the force-or-mortality in Eq. (6.1.1), the formulation for ${ }_{T} p_{x}(\vec{\kappa})$ will be

$$
\begin{aligned}
{ }_{T} p_{x}(\vec{\kappa}) & =\prod_{j=0}^{T-1} p_{x+j}\left(\kappa_{j}\right) \\
& =\exp \left(-\sum_{j=0}^{T-1} \mu_{x+j}\left(\kappa_{j}\right)\right) \\
& =\exp \left(-\sum_{j=0}^{T-1} \exp \left(\alpha_{x+j}+\beta_{x+j} \kappa_{j}\right)\right)
\end{aligned}
$$

As $N_{x}(t)=N_{x}(0) \times{ }_{t} p_{x}$ and the initial cohort $N_{x}(0)$ is known, $N_{x}(t)$ can be easily generated by using the simulation path of $\kappa_{t}$ process and calculating the survival probability with the Equation above. As a result, we will have a $n \times(T+1)$ matrix containing $n$ paths of $N_{x}(t)$.

To simulate $r_{P}^{(h)}(t)$, the core item is the technical component $B E_{0}\left(N_{x}(T)\right) / B E_{t}\left(N_{x}(T)\right)$ and the best-estimate value via the conditional expectation of $N_{x}(T)$ given the underlying processes/information at time $t$. Assuming $\kappa_{t}$ and $N_{x}(t)$ as Markov processes, they contain the full information up to and including time $t$. We use the shorter notation $\mathbb{E}_{t}\left[N_{x}(T)\right]$ instead of $\mathbb{E}\left[N_{x}(T) \mid N_{x}(t)\right]$. We assume that we have the estimated values of the agespecific parameters $a_{x}$ and $b_{x}$ in the Lee-Carter model for all possible ages.

\footnotetext{
${ }^{8}$ In case the actuarial risk $\kappa_{t}$ is independent of the financial risks $A_{t}$ and $r_{t}$, the simulation will be reduced to a two-dimensional Brownian motion and an independent one.
} 
The numerical implementation should be consistent with the implementation of the backward iteration of the one-period valuation. In the simulation stage above, each of the $n$ realization of $N_{x}(T)$ is built by one sequence of the underlying process $\kappa_{t}$ (or $N_{x}(t)$ ) that forms a random path for $N_{x}(t)$ over time. To establish the backward iteration for each typical time-step $(t-1, t)$, for $n$ realization of the state variable (e.g., $N_{x}(t)$ ), the numerical procedure must deliver another $n$ realization of the calculated value (e.g., $E_{t-1}\left[N_{x}(t)\right]$ ) that can be later used as the input for the time-step $(t-2, t-1) \cdot{ }^{9}$ Later, we will discuss this method in Section 6.3. Note that, we also need $n$ realization of $E_{t-1}\left[N_{x}(t)\right]$ to deliver $n$ replications of $r_{P}^{(h)}(t)$ and $P_{t}^{(h)}$.

We start from time $t=0$, where the expectation of the number of final survivors can be easily obtained by substituting the best-estimate of $\kappa_{t}$, i.e., $\mathbb{E}\left[\kappa_{t} \mid \kappa_{0}\right]=\kappa_{0}+\mu_{\kappa} t$ into Eq. (6.3.2) as a special $\kappa_{j}$ value. The simpler method is taking an average of $N_{x}(T)$ that implicitly is generated with information available at time $t=0$. We move to $t=1$, where in the numerical implementation we have $n$ realization of $N_{x}(T)$, and given $n$ values of $\kappa_{1}$ (or $N_{x}(1)$ ), we have to deliver $n$ values of $\mathbb{E}_{1}\left[N_{x}(T)\right]$. This means that for each given value of the underlying Markov process $\kappa_{1}$ or $N_{x}(1)$, we only have one realization of $N_{x}(T)$, while we need at least two observations to be able to calculate the average as the estimation of the conditional expectation. ${ }^{10}$ The efficient solution of this problem is delivered by the regression-based methods in the next subsection.

\section{Regression-based Methods}

Once again we use the regression-based techniques to implement the suitable numerical method to calculate the time-consistent and market-consistent price. Let $h\left(T, \kappa_{T}\right)$ be the payoff function contingent on $\kappa_{T}$. The target function is $f\left(t, \kappa_{t}\right)=\mathbb{E}\left[h\left(T, \kappa_{T}\right) \mid \kappa_{t}\right]$ for $0 \leq t \leq T$. We assume that the payoff $h$ is a square-integrable random variable given by $L^{2}\left(\Omega, \mathscr{G}_{t}, \mathbb{P}\right)$. The conditional expectation (i.e., $f\left(t, \kappa_{t}\right)$ can be obtained by an infinite series of the basis functions of $\kappa_{t}$

$$
\mathbb{E}\left[h\left(T, \kappa_{T}\right) \mid \kappa_{t}\right]=f\left(t, \kappa_{t}\right)=\sum_{k=0}^{\infty} a_{k} e_{k}\left(\kappa_{t}\right)
$$

with $e_{k}$ as the basis functions of the underlying risk driver at time $t<T$ and coefficients $a_{k} \in \mathbb{R}$.

The function $f$ can be estimated by a finite number of terms $K$ in the above summation as

$$
f\left(t, \kappa_{t}\right) \approx f_{K}\left(t, \kappa_{t}\right)=\sum_{k=0}^{K} a_{k} e_{k}\left(\kappa_{t}\right)
$$

\footnotetext{
${ }^{9}$ In particular, for the time-step $(0,1)$, we only need one value out of $n$ realizations as the backward iteration will not go further back.

${ }^{10}$ Other values of $N_{x}(T)$ are constructed based on the other value of $\kappa_{t}$ or $N_{x}(t)$.
} 
where the least-square estimator of $f\left(t, \kappa_{t}\right)$, denoted by $\widehat{f}$, can be calculated as a function of the least-square estimation of the vector of the regression coefficients $\widehat{\vec{a}}_{K}$ under the following argument,

$$
\widehat{\vec{a}}_{K}=\underset{\vec{a}_{K}}{\operatorname{argmin}} \sum_{i=1}^{n}\left[f_{i}\left(t, \kappa_{t}\right)-\sum_{k=0}^{K} a_{k} e_{k}\left(\kappa_{t}(i)\right)\right]^{2}
$$

where $f_{i}$ and $\kappa_{t}(i)$ denote the $i$ th realization of $f$ and $\kappa_{t}$ and $n$ is the total number of realizations.

The Eqs. (6.3.4) and (6.3.5) formulate a regression-based model to estimate the target function known as the "Least-Square Monte-Carlo (LSMC)" and/or "regression-now." The regression methods and the corresponding basis functions were proposed by Madan and Milne (1994) and later by Carriere (1996). Longstaff and Schwartz (2001) use this method to price American options. Glasserman and Yu (2004b) introduce another version of the regression-based methods called "regression-later estimator." In the regresslater method, one should first fit the payoff function with the underlying risk driver, both at time $T$ and then (using the additivity property of the expectation, take the expectation operator into the summation and) substitute for the analytical solution of the conditional expectation (if available). Unlike the regression-now method, regression later is modelindependent since by changing the pricing measure the regression coefficient and the fitting function does not change.

The both regression-based estimators are consistent with path-dependence of the hybrid policy reserve $P_{t}^{(h)}$, and possess key feature to operate corresponding the backward iteration procedure. Using the regression coefficients over each time-step, they can deliver a prediction of the target function (response variable) with $n$ replications from a vector of payoff (independent variable) with $n$ replications.

Now, we calculate $\mathbb{E}_{t}\left[N_{x}(T)\right]$ with the regression-based method in Subsection 6.3. For a general case of $0<t<T$, suppose that $N_{x}(T)$ can be estimated via polynomial basis functions of the underlying risk driver $\kappa_{T}$,

$$
\widehat{N}_{x}(T)=\sum_{k=0}^{K} \hat{a}_{k} \kappa_{T}^{k}
$$

Taking the conditional expectation, given $\kappa_{t}$, we have the estimation of the expectation of the conditional survivors as

$$
\begin{aligned}
\widehat{\mathbb{E}}\left[N_{x}(T) \mid \kappa_{t}\right] & =\mathbb{E}\left[\sum_{k=0}^{K} \hat{a}_{k} \kappa_{T}^{k} \mid \kappa_{t}\right] \\
& =\sum_{k=0}^{K} \hat{a_{k}} \mathbb{E}\left[\kappa_{T}^{k} \mid \kappa_{t}\right]
\end{aligned}
$$


However, as $\kappa_{T}$ is normally distributed, one can easily have the analytical solution of $\mathbb{E}\left[\kappa_{T}^{k} \mid \kappa_{t}\right]$. This is an application of the regression-later estimation for $\mathbb{E}_{t}\left[N_{x}(T)\right]$ and can be repeatedly used in the backward iteration. As the fitting function and regression coefficients remains the same, the $R^{2}$ of the regression should be very high for thsi estimation, so that we make sure the payoff $\left(N_{x}(T)\right)$ is fitted very well and is strongly close to its real values.

Another alternative to estimate $\mathbb{E}_{t}\left[N_{x}(T)\right]$ is using the regression-now method with either of the following models,

$$
\widehat{\mathbb{E}}\left[N_{x}(T) \mid \kappa_{t}\right]=\sum_{k=0}^{K} \hat{a}_{k} \kappa_{t}^{k}
$$

where $\kappa_{t}$ is used directly as the underlying risk driver to directly estimate $\mathbb{E}_{t}\left[N_{x}(T)\right]$, or

$$
\widehat{\mathbb{E}}\left[N_{x}(T) \mid \kappa_{t}\right]=\sum_{k=0}^{K} \hat{b_{k}} N_{x}^{k}(t) .
$$

where $\hat{b_{k}}$ is the estimation of the new regression coefficients for the regressors $N_{x}^{k}(t)$.

Note that, based on Eq. (6.3.2), $N_{x}(t)$ is by construction a function of all $\kappa_{j}$ values for $0<j<t$ over $(0, T)$, while $\kappa_{t}$ only reflects part of the available information to estimate $N_{x}(T)$. The same reasoning applies to the regression-later formula in Eq. (6.3.6), where $\kappa_{T}$ as the independent variable in the polynomial only possesses the last piece of the information used for $N_{x}(T)$. In this sense, we think the regression-now method in Eq. (6.3.10) is superior to the other options mentioned here. However, in a partitioned time period $t \in 1,2, \ldots, T$, to estimate the first conditional expectation $\mathbb{E}_{t=1}\left[N_{x}(T)\right]$, the regress-now estimator in Eq. (6.3.10) is not applicable as we have a single value for $N_{x}(0)$. For $t=1$, we will exceptionally use the regression-later estimator that does not have the above-mentioned shortage, and $\kappa_{1}$ provides the full information needed to calculate $N_{x}(1)$. Therefore, in this chapter, we use a combination of the regression-now and regression-later methods to calculate $T$ vector of the technical component of the hybrid profit-sharing mechanism over the contract term.

Now, we have all the necessary materials in Eq. (6.1.15) to simulate $r_{P}^{(h)}(t)$ and $P_{t}^{(h)}$. At time $t=1$, we use $A_{0}$ and $P_{0}$ to calculate $r_{P}^{(h)}(1)=\max \left[r_{G}, \alpha\left(A_{0} / P_{0}-(1+\gamma)\right)\right]^{11}$ and the vector $P_{1}^{(h)}=P_{0} \times r_{P}^{(h)}(1)$. At time $t=2$, we use the simulated vector of $A_{1}$, the vector $P_{1}^{(h)}$ we obtained in the previous period and the vector of $\mathbb{E}_{0}\left[N_{x}(T)\right] / \mathbb{E}_{1}\left[N_{x}(T)\right]$ that we estimated earlier by the regression-based methods. Using Eq. (6.1.15), we calculate $r_{P}^{(h)}(2)$ and $P_{2}^{(h)}$ and continue to do so until time $T$. By the simulated sequential vectors up to

\footnotetext{
${ }^{11}$ Note that at $t=1$, the technical component, $\frac{\mathbb{E}_{0}\left[N_{x}(T)\right]}{\mathbb{E}_{t-1}\left[N_{x}(T)\right]}$ is equal to one.
} 
$r_{T}, N_{x}(T)$, and $P_{t}^{(T)}$, we can calculate the payoff in Eq. (6.1.12) and start to calculate the pricing operators.

\section{Price Calculation by LSMC}

The Standard-Deviation actuarial price is made of the first two moments of the discounted payoff. Therefore, we can use the LSMC to implement the estimation of the price operator. We use the same method that we introduced in Chapter 5 with two underlying risk drivers (one financial and one actuarial). However in this section, we have one more financial risk driver, and hence, we extend the method to use three risk drivers. $r_{t}$ and $A_{t}$ are the underlying financial risk drivers and $N_{x}(t)$ is the actuarial risk driver. ${ }^{12}$ We start from the maturity $T$ as the beginning point of the backward iteration, and apply the twostep Standard-deviation operator in Eq. (6.2.3) over the interval $(T-1, T)$. The discounted payoff is

$$
f_{T}^{(h)}=\exp \left(-\int_{T-1}^{T} r_{s} \mathrm{~d} s\right) P_{T}^{(h)} \times N_{x}(T)
$$

where the discounting factor is the price of the zero-coupon bond issued on $T-1$ with maturity on $T$.

Suppose, the valuation period $(0, T)$ is partitioned as $\{0,1,2, \ldots, T\}$ and we have the simulated paths over the partitioned times for $A_{t}, r_{t}, N_{x}(t)$, and $P_{t}^{(h)}$ with $n$ replications. The pricing procedure is as follows,

- Start from maturity $T$ and value over $(T-1, T)$. In the inner step, the conditional expectation of the payoff and the squared payoff, can be estimated through the following regressions,

$$
\begin{aligned}
& \widehat{\mathbb{E}^{\mathbb{P}}}\left[\exp \left(-\int_{T-1}^{T} r_{s} \mathrm{~d} s\right) P_{T}^{(h)} \times N_{x}(T) \mid A_{T}, r_{T}, \kappa_{T-1}\right]=\sum_{k=0}^{K-1} \widehat{a}_{k}^{(1, T)} e_{k}\left(A_{T}, r_{T}, \kappa_{T-1}\right) \\
& \widehat{\mathbb{E}^{\mathbb{P}}}\left[\left(\exp \left(-\int_{T-1}^{T} r_{s} \mathrm{~d} s\right) P_{T}^{(h)} \times N_{x}(T)\right)^{2} \mid A_{T}, r_{T}, \kappa_{T-1}\right]=\sum_{k=0}^{K-1} \widehat{a}_{k}^{(2, T)} e_{k}\left(A_{T}, r_{T}, \kappa_{T-1}\right)
\end{aligned}
$$

where the regression coefficients ${\widehat{a_{k}}}^{(1, T)}$ and ${\widehat{a_{k}}}^{(2, T)}$ are estimated under the argument of minimizing the squared error.

\footnotetext{
${ }^{12}$ We prefer $N_{x}(t)$ to $\kappa_{t}$ as it captures, by construction, the whole information over the path up to time $t$ and is more informative than $\kappa_{t}$.
} 
- Calculate the Conditional premium $\pi^{\mathrm{s}}\left(A_{T}, r_{T}, \kappa_{T-1}\right)$ in the inner step,

$$
\pi^{\mathrm{S}}\left(A_{T}, r_{T}, \kappa_{T-1}\right)=\widehat{\mathbb{E}^{\mathrm{P}}}\left[f_{T}^{(h)}\right]+\beta \sqrt{\widehat{\mathbb{E}^{\mathbb{P}}}\left[\left(f_{T}^{(h)}\right)^{2}\right]-\left(\widehat{\mathbb{E}^{\mathrm{P}}}\left[f_{T}^{(h)}\right]\right)^{2}}
$$

This value is the result of the inner step and is used as the payoff for the outer step.

- We apply the outer step and estimate the arbitrage-free price (i.e., the conditional expectation) with the following regression equation,

$$
\begin{aligned}
\pi^{\mathrm{S}}\left(A_{T-1}, r_{T-1}, \kappa_{T-1}\right) & \left.=\widehat{\mathbb{E}^{\mathrm{Q}}}\left[\pi^{\mathrm{s}}\left(A_{T}, r_{T}, \kappa_{T-1}\right)\right) \mid A_{T-1}, r_{T-1}, \kappa_{T-1}\right] \\
& =\sum_{k=0}^{K-1}{\hat{b_{k}}}^{(T)} e_{\pi^{\mathrm{s}}}\left(A_{T-1}, r_{T-1}, \kappa_{T-1}\right)
\end{aligned}
$$

where again $b_{k}^{(T)}$ is estimated by minimizing the squared error. Note that going backward in time, $\pi^{\mathrm{s}}\left(A_{T-1}, r_{T-1}, \kappa_{T-1}\right)$ now will be used as a new payoff vector at time $T-1$ to implement the two-step valuation over $(T-2, T-1)$ and calculate the value at $T-2$.

- Repeat the above three steps, for all typical time steps of the form $(t, t+1)$, going backward in time over the partitioned valuation period up to time zero.

\subsection{Numerical Results}

In this section, we provide the results of the numerical methods described in the previous section to calculate the time-consistent two-step actuarial price of the participating policy.

We obtain the price as a single premium for a participating policy with a single payoff at maturity. The initial cohort is assumed to be $N_{x}(0)=100,000$ equal males and females with the age of $x=40$ years at the start of the contract, and different maturities are set up to $T=30$ years (with main focus on $5,10,20$, and 30 years). We calibrate the Lee-Carter model by the Dutch aggregated mortality tables for men and women during 1960-2006 (47 years) and obtain the parameters of the mortality trend process $k_{t}$ as follows: The initial value $\kappa_{0}=-24.5637$, the drift $\mu_{\kappa}=-0.8089$, and the diffusion coefficient $\sigma_{\kappa}=1.4734$.

To calibrate the Hull-White interest rate model, we use a 30 -year annual zero rates in the Euro area released by the European Central Bank (ECB) on January 2, 2006. ${ }^{13}$ The rates are calculated based on the prices of the AAA-rated euro area central government bonds. We set the mean reversion parameter of the Hull-White model as $a=0.04$ and work with a variety of the volatility parameter $\sigma_{r}$ between $(0.01,0.1)$.

\footnotetext{
${ }^{13}$ Accessible from ECB webpage, https://www.ecb.europa.eu/stats/money/yc/html/index.en.html.
} 
We work with the Monte-Carlo simulation of $n=1,000$ scenario paths for the underlying risk drivers. The number of paths remains constant for implementation of the regression-based methods for every time-step in the backward iteration. We then repeat the simulation $N=100$ times, which gives us the standard error and the confidence interval around the estimated price.

\section{Time-Consistent and Market-Consistent Price}

We simulate the hybrid payoff in Eq. (6.1.12) using the method described in Subsection 6.3, and calculate the time-consistent two-step Standard-Deviation actuarial price in Eq. (6.2.3) based on the simulation of the policy interest rate in Eq. (6.1.15). In Eq. (6.1.7), we set the correlation of the financial risk drivers $\rho_{A, r}=0.25$ and assume the correlation of $\kappa_{t}$ with $A_{t}$ and $r_{t}$ equal to zero. Later, we will study the effect of the correlation on the time-consistent and market-consistent price.

As we required both time-consistency and market-consistency for the desirable price, it is important to compare our Standard-Deviation actuarial price with the other alternative time-consistent market-consistent price, which is the two-step expectation operator. ${ }^{14}$ As we mentioned before, the expectation operator is intrinsically time-consistent due to the tower property. Thus, we do not need to apply the backward iteration over the valuation period and we only use one-period valuation method. Thus, both values are time-consistent and market-consistent.

Figure 6.2, exhibits the result of the time-consistent and market-consistent StandardDeviation actuarial price and the one-period expectation price operator for different maturities of $T=\{1,2, \ldots, 30\}$. The difference of the two values is the time-consistent and market-consistent actuarial risk-loading that is added to the expectation to penalize for the possible future unexpected losses. The risk-loading is small for the short-term contracts $(0.2 \%$ for a 5 -years contract), but increases when the maturity is longer $(6.2 \%$ for a 25 -years and $8.9 \%$ for a 30 -years contract). We observe a reasonable $95 \%$ confidence interval for the price, where for the longest maturity (30-years) the lower/upper bound has $0.7 \%$ difference with the point estimation.

In Chapter 4, we have discussed the "time-consistency risk premium" as the difference between the one-period and time-consistent (multi-period) two-step actuarial prices. The one-period actuarial price is formulized by adding a portion of the standard deviation to the expected value of the discounted payoff (also one-period) in Eq. (6.2.2), while the timeconsistent price comes from Eq. (6.2.3). We call the surplus of the one-period standard deviation price to the expectation "the one-period risk-loading." Therefore, as a matter of clarification, the surplus of the one-period Standard-Deviation price over the expected

\footnotetext{
${ }^{14}$ This means that in the inner step, we omit the loading part of the Standard-Deviation principle and only keep the expectation.
} 

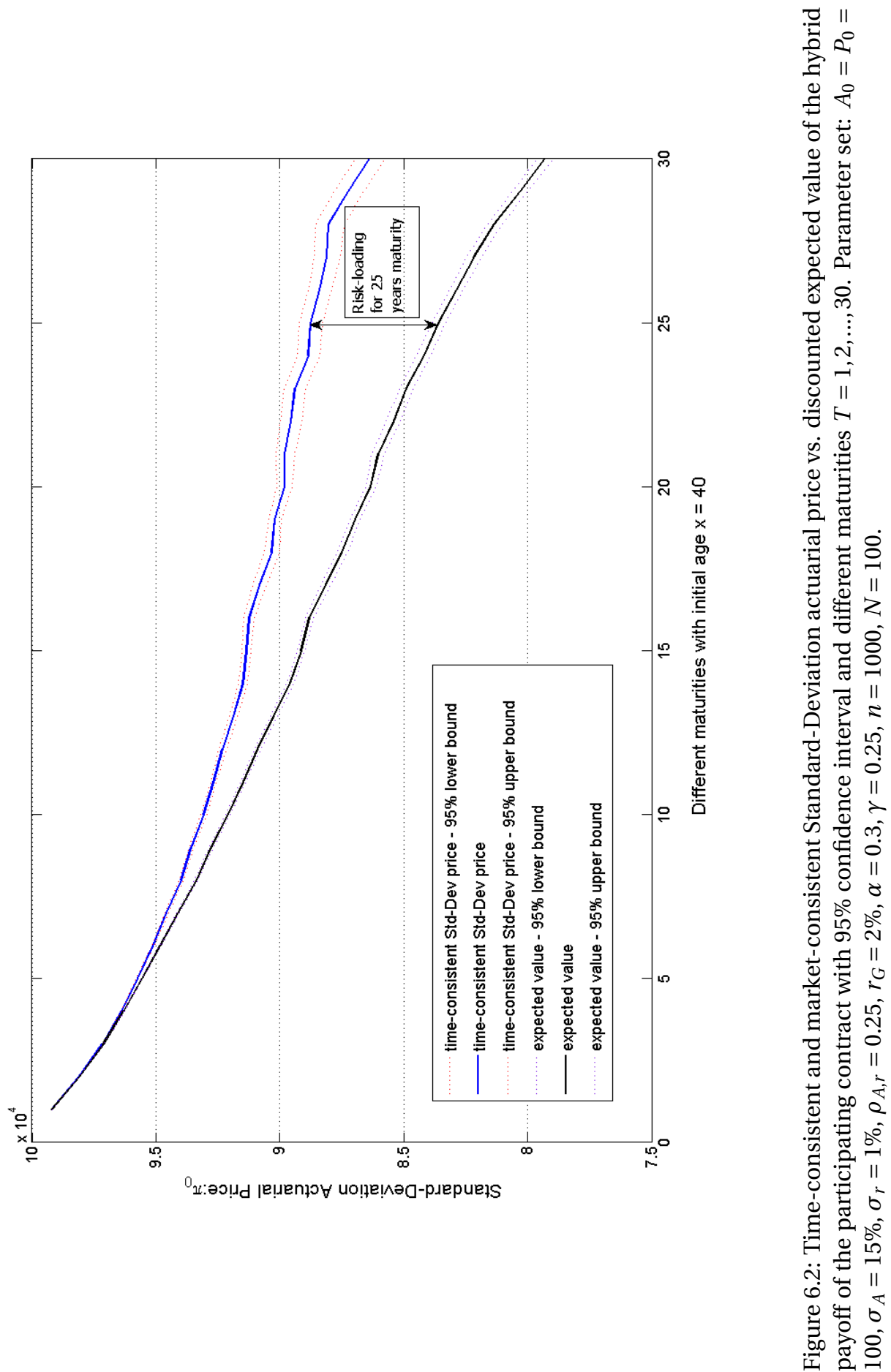
Table 6.1: Values of the participating contract with different maturities for the initial cohort of $N_{40}(0)=1000$ and the ratio of the one-period risk-loading and time-consistency risk premium on top of the expected value of the contract.

\begin{tabular}{lcccccc}
\hline \multicolumn{7}{c}{$\mathrm{T}$} \\
\hline Two-step Price & 5 & 10 & 15 & 20 & 25 & 30 \\
\hline Expected-value & $95,558.6$ & $92,088.3$ & $89,138.6$ & $86,328.2$ & $83,600.2$ & $79,284.6$ \\
One-period Std-Dev & $95,574.6$ & $92,123.6$ & $89,198.5$ & $86,422.7$ & $83,746.6$ & $79,513.5$ \\
Time-Consistent Std-Dev & $95,752.7$ & $93,072.1$ & $91,342.2$ & $89,802.1$ & $88,761.6$ & $86,365.5$ \\
One-period Risk-loading & $0.02 \%$ & $0.04 \%$ & $0.07 \%$ & $0.11 \%$ & $0.19 \%$ & $0.31 \%$ \\
Time-consistency Premium & $0.19 \%$ & $1.03 \%$ & $2.40 \%$ & $3.91 \%$ & $5.99 \%$ & $8.62 \%$ \\
Total Risk-loading & $0.20 \%$ & $1.07 \%$ & $2.47 \%$ & $4.02 \%$ & $6.17 \%$ & $8.93 \%$ \\
Ratio of TC Premium & $91.8 \%$ & $96.4 \%$ & $97.2 \%$ & $97.2 \%$ & $97.0 \%$ & $96.5 \%$ \\
\hline
\end{tabular}

Parameter set: $A_{0}=P_{0}=100, \sigma_{A}=15 \%, \sigma_{r}=1 \%, \rho_{A, r}=0.25, r_{G}=2 \%, n=1000, N=100$.

value is the one-period risk-loading, and the surplus of the time-consistent standarddeviation price over the one-period Standard-deviation price is the "time-consistency risk premium" that together the sum up to the risk-loading computed in the previous paragraph.

Risk-loading = One-period risk-loading + Time-consistency risk premium

Since we emphasize the requirement of time-consistency with a market-consistent price, it is interesting to know how the risk-loading will be partitioned between the timeconsistency risk premium and the one-period risk-loading.

Table 6.1 shows that for all maturities, more than $90 \%$ of the risk-loading is the timeconsistency risk premium and only a small portion is made of the one-period StandardDeviation loading. Note that for each maturity, the result is obtained out of an annual backward iteration of the two-step valuation along the valuation period until time zero. None of the longer maturity prices are obtained by using the values of the shorter maturities.

In general, different factors can affect the price of the participating contract with a hybrid payoff and crediting (profit-sharing) mechanisms. Among all factors, when the maturity increases, the asset value increases and the discount factor and the number of survivors decreases, on average. We also have the one-period risk-loading and the timeconsistency risk premium that increases in maturity and can enlarge the price over time. Among the above mentioned factors, in Figures 6.2, we observe that the discount factor and the number of survivors dominate the others and decrease the price when the maturity increases. Moreover, regardless of the maturity, recalling Eq. (6.1.15), the other effective factors are the distribution ratio $(\alpha)$, the volatility of the investment asset $\left(\sigma_{A}\right)$, and 
the volatility of the interest rate $\left(\sigma_{r}\right)$ that positively affects price, and the target buffer ratio $(\gamma)$ that negatively affects price. See (Grosen and Jorgensen, 2000) for an explanation of. Higher $\alpha$ means a more liberal profit sharing policy of the fund managers, while a higher $\gamma$ means that the fund managers follow a rather conservative policy for profit sharing and the policyholders can get part of the excess investment return on top of the guaranteed rate $r_{G}$ only if the funding ratio is very high.

In Tables 6.4 we study the effect of the distribution ratio $(\alpha)$, the target buffer ratio $(\gamma)$, and the volatility of the investment asset $\left(\sigma_{A}\right)$ on the price of the contract. For each $\sigma_{A}, \alpha$, $\gamma$, and maturity chosen from the set $T \in\{5,10,15,20,25,30\}$, we provided the price of the contract and summarize the average standard error of the price for different amounts of $\gamma$ obtained from the Monte-Carlo simulation. The table is divided into two vertical panels, each of which provides the results for two levels of the investment asset volatility. In the left panel the prices and standard errors are provided for $\sigma_{A}=15 \%$, while in the right one the results are reflected for $\sigma_{A}=30 \%$. There are also horizontal panels where each panel represents the prices and average Standard error for a special level of the distribution ratio $(\alpha)$. Within each horizontal panel, the prices and standard errors vary for different levels of the target buffer ratio $(\gamma)$ and maturities $(T)$.

We first consider the first panel where $\alpha=0$ and no bonus/surplus over the guaranteed rate $r_{G}$ is distributed every year. This turns the participating contract into a "longevity bond" sold to a cohort of $N_{x}(0)$ and deactivates the hybrid crediting mechanism too. As there is no profit-sharing, and the overall term structure is higher than $r_{G}$, the price in this panel will be always below the par value $P_{0}=100$. As the crediting interest rate is always $r_{G}$ in this level of $\alpha$, the change in the volatility of the investment asset, plays no role on the price and its standard error, when we move to in this panel from $\sigma_{A}=15 \%$ to $\sigma_{A}=30 \%$. See that the right and left half of this panel are almost the same. When $\alpha$ rises, there will be a positive profit-sharing in the contract that is also called "bonus option." So the difference between the prices in the lower panels and the first panel (where $\alpha=0$ ), provides the price of the bonus option. Going down over the panels with higher $\alpha$, the bonus (profit-sharing) policy gets more liberal and increases the price of the bonus option.

When $\gamma=0$, it means that he fund managers do not hold any buffer reserve for the fund, and as long as the assets are over the policy-reserves, the policy holders will have a chance to receive returns. Considering the right columns of each panel over higher amounts of $\gamma$, the bonus (profit-sharing) policy of the fund managers gets more conservative and the price of contract decreases, and a lower price is reported for the bonus option.

In the lower panels, when the higher distribution ratio allows for more freedom to achieve higher crediting interest rate, the price of the bonus option (and therefore the price of the contract) increases for all maturities as expected. On the other hand, when the investment asset becomes more volatile and we increase the volatility from $15 \%$ to $30 \%$ in the right half, the prices also increase. The price growth due to a change of the volatil- 
Table 6.2: Time-consistent and market-consistent actuarial Standard-Deviation price of the participating contract for different levels of the investment asset volatility $\sigma_{A}=15 \%$ and $\sigma_{A}=30 \%$, different profit-sharing mechanism parameters, $\alpha$ and $\gamma$, and different maturities.

\begin{tabular}{|c|c|c|c|c|c|c|c|c|c|c|c|c|}
\hline \multirow[b]{2}{*}{$\mathrm{T}$} & \multicolumn{5}{|c|}{$15 \%$} & \multirow[b]{2}{*}{$\begin{array}{l}\text { Average } \\
\text { SE }\end{array}$} & \multirow[b]{2}{*}{$\begin{array}{l}\gamma \\
0\end{array}$} & \multirow[b]{2}{*}{0.1} & \multicolumn{2}{|c|}{$30 \%$} & \multirow[b]{2}{*}{0.4} & \multirow[b]{2}{*}{$\begin{array}{c}\text { Average } \\
\text { SE }\end{array}$} \\
\hline & $\begin{array}{l}\gamma \\
0\end{array}$ & 0.1 & 0.2 & 0.3 & 0.4 & & & & 0.2 & 0.3 & & \\
\hline$\alpha=0.00$ & & & & & & & & & & & & $\times 10^{3}$ \\
\hline 5 & 94.26 & 94.27 & 94.26 & 94.25 & 94.26 & 0.006 & 94.26 & 94.27 & 94.27 & 94.26 & 94.26 & 0.006 \\
\hline 10 & 85.83 & 85.84 & 85.85 & 85.87 & 85.86 & 0.013 & 85.85 & 85.86 & 85.86 & 85.87 & 85.86 & 0.013 \\
\hline 15 & 76.94 & 76.88 & 76.89 & 76.94 & 76.91 & 0.021 & 76.91 & 76.93 & 76.92 & 76.91 & 76.89 & 0.021 \\
\hline 20 & 68.46 & 68.42 & 68.46 & 68.45 & 68.43 & 0.027 & 68.43 & 68.45 & 68.42 & 68.45 & 68.41 & 0.027 \\
\hline 25 & 60.37 & 60.35 & 60.34 & 60.37 & 60.40 & 0.031 & 60.31 & 60.37 & 60.32 & 60.36 & 60.30 & 0.030 \\
\hline 30 & 52.41 & 52.56 & 52.42 & 52.44 & 52.45 & 0.032 & 52.43 & 52.35 & 52.39 & 52.40 & 52.35 & 0.032 \\
\hline \multicolumn{13}{|l|}{$\alpha=0.25$} \\
\hline 5 & 100.05 & 97.98 & 96.59 & 95.74 & 95.21 & 0.022 & 107.60 & 105.02 & 103.10 & 101.71 & 100.52 & 0.068 \\
\hline 10 & 101.87 & 98.12 & 95.40 & 93.29 & 91.68 & 0.063 & 120.43 & 115.68 & 112.15 & 109.54 & 106.56 & 0.225 \\
\hline 15 & 103.29 & 98.52 & 94.91 & 91.81 & 89.47 & 0.114 & 133.28 & 127.66 & 123.48 & 117.31 & 114.58 & 0.509 \\
\hline 20 & 104.86 & 99.18 & 94.66 & 91.26 & 87.99 & 0.178 & 146.35 & 141.26 & 134.83 & 132.03 & 123.14 & 1.079 \\
\hline 25 & 106.55 & 100.58 & 95.40 & 90.92 & 87.37 & 0.254 & 162.18 & 157.46 & 149.20 & 139.81 & 133.20 & 1.485 \\
\hline 30 & 108.25 & 101.22 & 95.53 & 90.86 & 86.58 & 0.331 & 178.48 & 171.80 & 161.91 & 154.61 & 152.12 & 2.669 \\
\hline \multicolumn{13}{|l|}{$\alpha=0.50$} \\
\hline 5 & 105.62 & 101.70 & 99.05 & 97.41 & 96.28 & 0.036 & 118.06 & 114.04 & 110.61 & 107.69 & 105.42 & 0.115 \\
\hline 10 & 110.96 & 105.10 & 100.81 & 97.65 & 94.98 & 0.086 & 137.55 & 131.57 & 125.60 & 120.98 & 117.21 & 0.294 \\
\hline 15 & 114.26 & 107.49 & 102.12 & 98.04 & 94.77 & 0.150 & 156.39 & 148.97 & 141.29 & 133.64 & 129.02 & 0.675 \\
\hline 20 & 117.90 & 110.18 & 103.60 & 98.48 & 95.11 & 0.203 & 179.73 & 167.95 & 159.50 & 149.98 & 146.04 & 1.488 \\
\hline 25 & 121.00 & 112.51 & 105.36 & 100.05 & 95.26 & 0.303 & 200.93 & 186.44 & 177.99 & 169.76 & 159.04 & 2.667 \\
\hline 30 & 122.76 & 114.97 & 106.86 & 101.05 & 95.89 & 0.431 & 221.87 & 204.17 & 191.02 & 189.64 & 179.77 & 4.590 \\
\hline \multicolumn{13}{|l|}{$\alpha=0.75$} \\
\hline 5 & 109.57 & 104.51 & 100.90 & 98.67 & 97.19 & 0.045 & 126.31 & 120.38 & 115.85 & 112.19 & 109.08 & 0.146 \\
\hline 10 & 116.30 & 109.46 & 104.01 & 100.30 & 97.26 & 0.101 & 148.92 & 141.18 & 134.75 & 128.39 & 123.68 & 0.376 \\
\hline 15 & 120.71 & 112.65 & 106.56 & 101.60 & 97.81 & 0.167 & 170.79 & 160.18 & 153.34 & 144.43 & 138.92 & 0.723 \\
\hline 20 & 124.39 & 115.80 & 108.98 & 102.82 & 98.38 & 0.253 & 192.94 & 180.63 & 171.58 & 163.86 & 153.30 & 1.423 \\
\hline 25 & 127.58 & 118.55 & 111.08 & 104.00 & 99.42 & 0.294 & 221.98 & 204.01 & 193.13 & 175.89 & 172.74 & 2.616 \\
\hline 30 & 131.10 & 120.77 & 113.22 & 105.77 & 100.89 & 0.439 & 249.12 & 224.07 & 218.43 & 199.43 & 188.98 & 4.602 \\
\hline \multicolumn{13}{|l|}{$\alpha=1.00$} \\
\hline 5 & 112.62 & 106.47 & 102.36 & 99.71 & 97.89 & 0.057 & 132.43 & 125.54 & 119.94 & 115.73 & 112.18 & 0.150 \\
\hline 10 & 120.25 & 112.51 & 106.62 & 102.20 & 99.00 & 0.103 & 158.97 & 148.17 & 140.54 & 134.00 & 129.29 & 0.396 \\
\hline 15 & 124.74 & 115.95 & 109.41 & 103.94 & 99.83 & 0.167 & 182.01 & 168.92 & 161.26 & 152.57 & 145.70 & 0.807 \\
\hline 20 & 129.33 & 120.01 & 112.19 & 105.94 & 101.06 & 0.259 & 205.23 & 190.34 & 179.50 & 171.97 & 163.48 & 1.563 \\
\hline 25 & 133.28 & 122.71 & 115.29 & 108.41 & 102.45 & 0.334 & 234.10 & 220.18 & 204.77 & 193.60 & 182.59 & 2.870 \\
\hline 30 & 136.39 & 125.25 & 117.24 & 110.28 & 104.54 & 0.501 & 254.05 & 243.41 & 225.48 & 208.44 & 209.52 & 4.156 \\
\hline
\end{tabular}

Parameter set: $A_{0}=P_{0}=100, \sigma_{A}=15 \%, \sigma_{r}=1 \%, \rho_{A, r}=0.25, r_{G}=2 \%, n=1000, N=100$. 
ity is more dramatic for the longer maturities. Compare for example the numbers in the panel with $\alpha=0.50$ and $\gamma=0.2$. For the maturity $T=5$, the price goes from $99.05 \times 10^{3}$ for $\sigma_{A}=15 \%$ to $110.61 \times 10^{3}$ equivalent to $11.7 \%$ growth. However, if we consider the maturity $T=30$, the price increases from $106.86 \times 10^{3}$ to $191.02 \times 10^{3}$, exhibiting a $78.8 \%$ growth. In this table, the volatility of the Hull-White interest rate model $\left(\sigma_{r}\right)$ is fixed to $\sigma_{r}=0.05$. In Subection 6.4, we study the effect of the interest rate factor and its volatility on the price.

To have a better view of the effect of the funding policy over different maturities, we provide the above results for the time-consistent and market-consistent price over time. We compare the value of the contract with three combined levels of the distribution ratio, $\alpha$, and the funding ratio, $1+\gamma$ that represent three different funding policy scenarios, including:

- Precautionary policy, where we set $\alpha=25 \%$ and $1+\gamma=130 \%$,

- Moderate policy, where we set $\alpha=50 \%$ and $1+\gamma=115 \%$, and

- Liberal policy, where we set $\alpha=75 \%$ and $1+\gamma=105 \%$.

In the precautionary policy, for example, the fund management requires a relatively high funding ratio, $1+\gamma=130 \%$, and will distribute a relatively low portion, $\alpha=25 \%$ of the extra buffer on top of the guaranteed rate $r_{G}=2 \%$ as the bonus if that holds. The opposite happens with the liberal policy. The rest of the parameters stay unchanged with respect to Table 6.4.

The comparative values for the abovementioned funding policies and their evolution over different maturities are provided in Figure 6.3. The precautionary funding policy requires a lower risk premium and price as compared to the liberal and moderate policies. The positive effect of the maturity $T$ and the asset return volatility $\sigma_{A}$ is obviously reflected where higher maturities provide higher compensation against the effect of the discount factor, and prices with higher asset volatility dominate those with lower ones. The only exception is the case where the price under the liberal policy with $\sigma_{A}=15 \%$ is higher than the price under the precautionary policy with $\sigma_{A}=30 \%$ for maturities lower under $T=14$. If we compare the results over time, for example when $\sigma_{A}=15 \%$, the price under the liberal policy for a contract with maturity $T=15$ is approximately $26.6 \%$ higher than precautionary policy, while for a contract with $T=30$ years, this difference is around $40.3 \%$.

The reference value for the prices under any funding policy, is the price of the longevity bond when there is no profit sharing, shown with the thick blue line in the Figure 6.3. This price, as the guaranteed part of the liability, is the same for all funding policies (shown with the green and red lines) and decreases with longer maturities due to effect of the discount factor. The surplus over this value is the time-consistent and market-consistent price of the option element of the contract that increases for longer maturities. Comparing the 


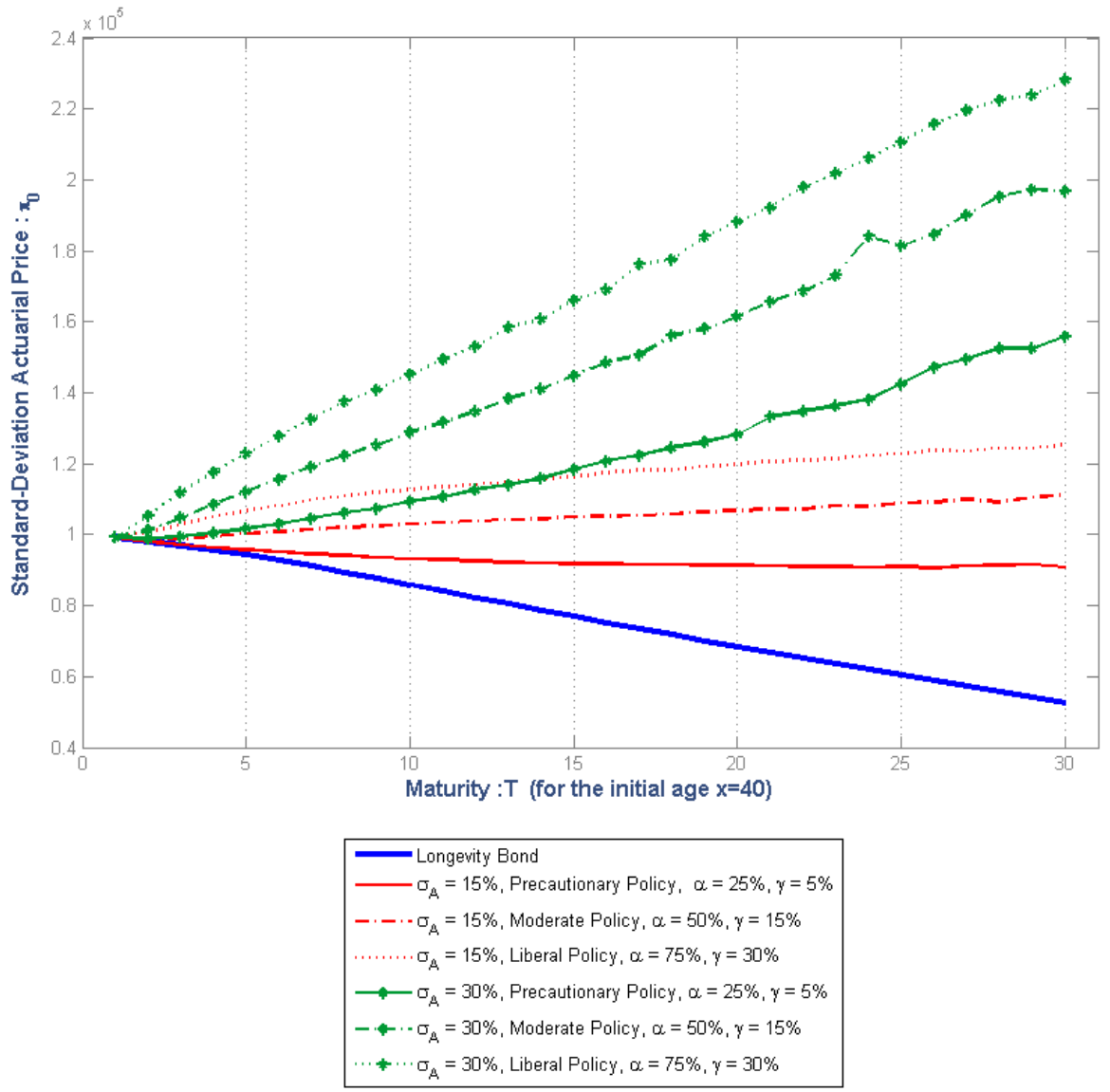

Figure 6.3: Comparing the effect of the funding policy on the time-consistent and marketconsistent Standard-Deviation of the participating contract, simulated under Precautionary, Moderate, and Liberal policies and two different level of the asset return volatility $\sigma_{A}=15 \%$ and $\sigma_{A}=30 \%$ over different maturities $T=\{1,2, \ldots, 30\}$. Other parameters: $A_{0}=P_{0}=100, \sigma_{A}=15 \%, \sigma_{r}=1 \%, \rho_{A, r}=0.25, r_{G}=2 \%, \alpha=0.3, \gamma=0.25, n=1000$, $N=100$. 
price for maturity $T=25$, and comparing to the price of the longevity bond equal to $60.35^{3}$, the price of the option element (surplus return on top of $r_{G}$ ) for $\sigma_{A}=15 \%$, adds $51 \%$ to the longevity bond price for precautionary policy and respectively $80 \%$ and $103 \%$ for moderate and liberal policy. Therefore, under the liberal policy, the time-consistent and marketconsistent price of the participating contract is more than double the price of the longevity bond. The price of the option element for the higher asset volatility $\sigma_{A}=30 \%$ adds $136 \%$, $200 \%$, and $249 \%$ to the longevity bond price under the precautionary policy, moderate policy, and liberal policy respectively. Thus, as we double the asset return volatility (increase $\sigma_{A}$ from $15 \%$ to $\left.30 \%\right)$, the price of the option element more than doubles $(2.49 \%)$. This shows that the option element of the contract is highly sensitive to the asset return volatility $\left(\sigma_{A}\right)$. This result holds for other maturities as well, which suggests that it this holds over time.

\section{The Effect of the Stochastic Interest Rate}

We assess the effect of the stochastic interest rate on the value of the contract by comparing the values obtained by the stochastic term-structure under the Hull-White interest rate model in Eq. (6.1.6) and the deterministic term-structure where in the same model $\sigma_{r}=0$. For the stochastic interest rate, we provide the values for three levels of the interest rate volatility $\sigma_{r}=\{0.01,0.05,0.1\}$ that, together with the deterministic rate, gives four different version of the price to compare. We compare the effect for the discounted expected value $^{15}$ and the time-consistent and market-consistent Standard-Deviation price. The rest of the parameters are fixed as mentioned in the beginning of this section.

Table 6.3: The percentage difference between the time-consistent and market-consistent Standard-Deviation price, modeled by the stochastic interest rate with three levels of $\sigma_{r}=$ $\{0.01,0.05,0.1\}$ in the Hull-White model and the deterministic interest rate, calculated for three long-dated participating contracts with maturities $T=\{20,25,30\}$.

\begin{tabular}{ccccccc}
\hline \multicolumn{3}{c}{ Expected Value } & \multicolumn{5}{c}{ TC \& MC Std-Dev Price } \\
\hline $\mathrm{T}$ & $\sigma_{r}$ & & \multicolumn{6}{c}{$\sigma_{r}$} \\
& 0.01 & 0.05 & 0.1 & 0.01 & 0.05 & 0.1 \\
\hline & & & & & & \\
$\mathrm{T}=20$ & $0.15 \%$ & $1.52 \%$ & $5.28 \%$ & $0.19 \%$ & $1.84 \%$ & $5.52 \%$ \\
$\mathrm{~T}=25$ & $0.24 \%$ & $2.62 \%$ & $10.13 \%$ & $0.29 \%$ & $3.12 \%$ & $11.05 \%$ \\
$\mathrm{~T}=30$ & $0.35 \%$ & $4.73 \%$ & $17.61 \%$ & $0.46 \%$ & $6.61 \%$ & $20.40 \%$ \\
\hline
\end{tabular}

Table 6.3 represents the percentage difference between the values obtained by the stochastic and deterministic interest rate. The left part of the table delivers the percentage

\footnotetext{
${ }^{15} \mathrm{As}$ a reminder: this value is by construction time-consistent and market-consistent.
} 


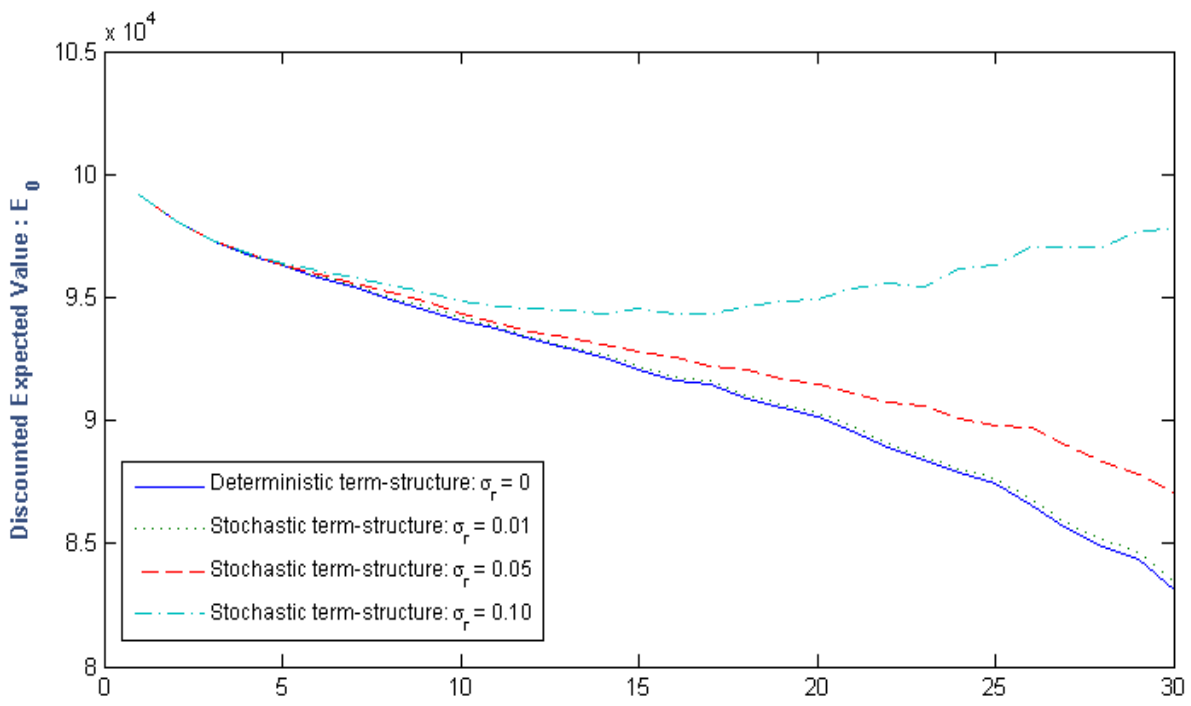

(A) Maturity of the contract: $T$, for the initial age $x=40$

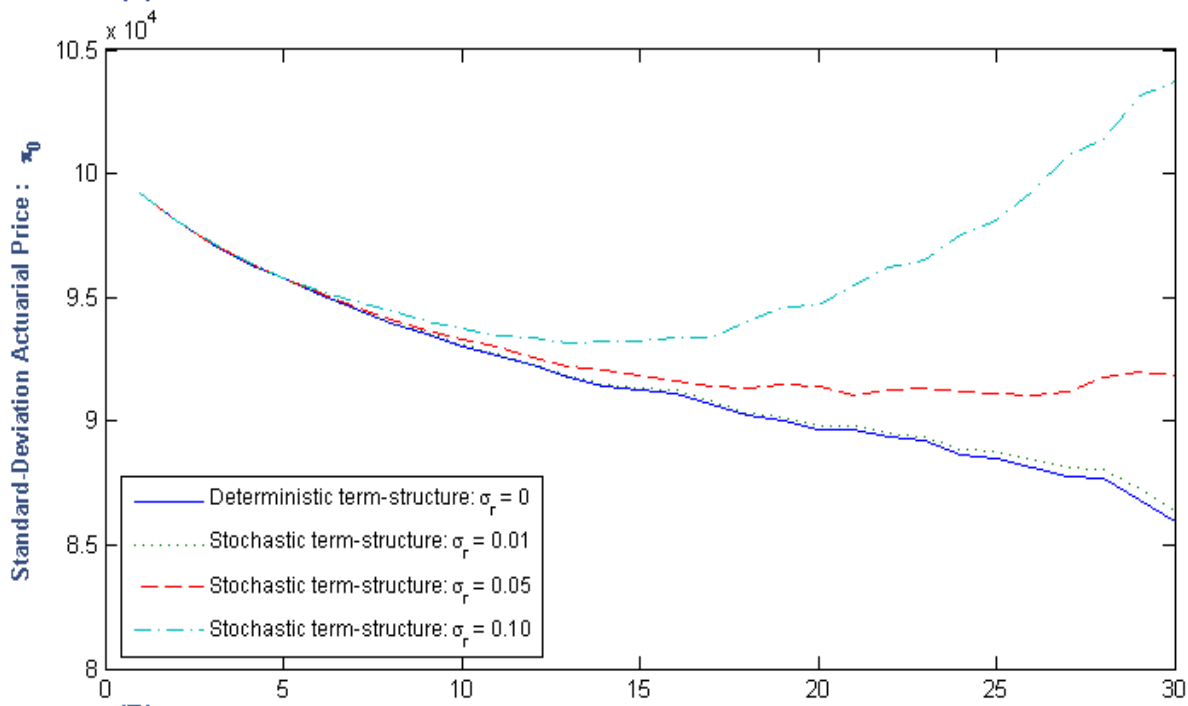

(B)

Maturity of the contract: $T$, for the initial age $x=40$

Figure 6.4: Comparing the effect of the stochastic and deterministic interest rate on the value of the participating contract, simulated with three different levels of the interest rate volatility $\sigma_{r}=\{0.01,0.05,0.1\}$ in the Hull-White model and $\sigma_{r}=0$ for the deterministic interest rate over different maturities $T=\{1,2, \ldots, 30\}$. Figure (A): Discounted expected value and figure (B): Time-consistent and market-consistent Standard-Deviation actuarial price. Other parameters set: $A_{0}=P_{0}=100, \sigma_{A}=15 \%, \sigma_{r}=1 \%, \rho_{A, r}=0.25, r_{G}=2 \%$, $\alpha=0.3, \gamma=0.25, n=1000, N=100$. 
difference for the discounted expected value while the right part represents the percentage difference for the Standard-deviation price. The differences are reported for three levels of $\sigma_{r}$ and three maturities $T=20,25,30$. In general, as all the observed percentage differences are positive, we conclude that the effect of the stochastic interest rate can be captured by a special risk-loading on top of the value obtained by deterministic interest rate; let us call it "interest rate loading." At first glance, comparing to the expected values (left part of the table), the time-consistent and market-consistent Standard-Deviation price (right part of the table) shows slightly higher interest rate loading on the price. We observe that the interest rate volatility $\sigma_{r}$ is the factor that causes the dramatic increase in the interest rate loading. For example for $T=25$, increasing $\sigma_{r}$ from 0.01 to 0.1 (10 times larger) causes the interest rate loading for the Standard-Deviation price to increase from $0.29 \%$ to $11.05 \%$ (around 38 times larger). However, generally for the lower level of $\sigma_{r}=0.01$, the interest rate loading for all maturities stays very small: between $(0.15-0.35) \%$ for the expected value and $(0.19-.46) \%$ for Standard-Deviation price. This is useful for practitioners as they can expect to be close to the reasonable price with stochastic interest rate (even if calculated with a deterministic term structure) if the market for the interest rate derivatives is not volatile and in their model $\sigma_{r}$ is estimated as a very small quantity. This significantly reduces the numerical algorithm required in the calculation phase. The interest rate loading also increases by the maturity $T$ for both the discounted expected value and Standard-deviation price. For example, the loading for the discounted expected value with $\sigma=0.05$ increases by $1.52 \%, 2.62 \%$, and $4.73 \%$ for $T=20, T=25$, and $T=30$, respectively. For the shorter maturities the interest rate loading is smaller. In general, the effect of the stochastic interest rate on the time-consistent market-consistent Standard-Deviation price is slightly higher than the discounted expected value, considering into change patterns with very similar sensitivity to interest rate volatility and maturity.

Note that the above results are based on a relatively conservative profit-sharing policies with $\alpha=0.3$ and $\gamma=0.25$, where the volatility of the crediting interest rate is by construction relatively smaller as compared to a moderate or liberal policy. Different profitsharing policies may lead to different levels of interest rate loading with different sensitivities to $\sigma_{r}$ and $T$.

Figure 6.4, shows the graphical evolution of price of the participating contract over 30 years of maturities. It represents two different values modeled with the stochastic and deterministic interest rate: (A) for the discounted expected value and (B) for the timeconsistent and market-consistent Standard-deviation price. One can observe that for the both values with maturities less than $T=5$ years, the randomness of the interest rate (with any level of the interest rate volatility $\sigma_{r}$ ) is negligible, and the difference is trivial up to $T=10$ years. However, when the maturity increases above ten years $(T>10)$, the prices obtained by the stochastic interest rate dominate the price obtained by the deterministic interest rate, although the difference between the $\sigma_{r}=0.01$ level and deterministic rate 
is still very little. The evolution of the value difference for the discounted expected value and Standard-Deviation price with the three levels of $\sigma_{r}$ looks similar, where the interest rate loading for the long-dated contracts with more volatile interest rate is more severe, while not being remarkable for the small $\sigma_{r}$. Clearly with the highly volatile interest rate in the long maturities, the Standard-Deviation price reflects higher interest rate loading as compared to the expected discounted value. 


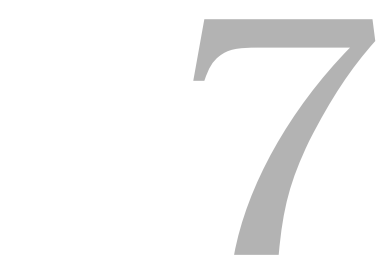

Conclusions

This chapter concludes this dissertation. We summarize in the order that chapters appeared. In the last part we share some ideas and questions for further research.

\subsection{Time-consistent Valuations}

We first investigated a number of well-known actuarial premium principles, such as the Variance and Standard-Deviation principle, and studied their extension into a timeconsistent direction. We constructed these extensions using one-period valuations, then we extended this to a multi-period setting by means of the backward iteration method of Jobert and Rogers (2008) for a given discrete time-step $\Delta t$, and finally we considered the continuous-time limit for $\Delta t \rightarrow 0$. We showed that the extended Variance premium principle converges to the non-linear exponential indifference valuation. Furthermore, we showed that the extended Standard-Deviation principle converges to an expectation under an equivalent martingale measure. Finally, we showed that the Cost-of-Capital principle, which is widely used by the insurance industry, converges to the same limit as that of the Standard-Deviation principle. In the above cases, we assumed that the underlying risk process is a simple diffusion process in which the continuous-time limit of the timeconsistent valuation results in a semi-linear Partial Differential Equation (PDE) that can be solved analytically with the Feynman-Kaç formula. To conduct a more realistic valuation, we added a Poisson jump component to the underlying risk process and obtained the time-consistent extension of the above premium principles in the form of different Partial Integro-Differential Equations (PIDEs) that can be solved numerically. There was no convergence in the price of the different premium principles in the jump case, but the effect of the jump component is reflected in the related PIDEs by different forms of premium jumps. In the Cost-of-Capital principle, the $\mathrm{VaR}_{1-q}$ operator failed to reflect the effect of the jump on the extended price where the probability of the jump in a single time step drops to less than the probability level of the quantile, $\lambda \Delta t<q$. This uncovers an important weakness of the Cost-of-Capital principle in the time-consistent extension, to price 
the insurance risks that contains the jump components. The end of the Chapter 3 is dedicated to use the Markov chain approximation to apply the backward iteration method and calculate the time-consistent price. Here, we observed the convergence of the numerical calculation to the analytical time-consistent solutions.

\subsection{Market-consistent Valuations}

This thesis makes two main contributions on the market-consistent valuation. First, we provided an implementation of the two-step valuation method. Second, we applied this implementation to price a stylized life insurance contract in a market-consistent manner. We first applied the method in a one-period valuation setting and provided insights into how the market-consistent price differs from the usual price. To address usage in the Solvency II and EIOPA frameworks, we provided a representation of the two-step valuation for the EIOPA risk-margin method and time-consistent operator built through backward iteration of the one-period two-step actuarial valuation for the risk margin. Both methods are implemented in multi-period settings in which we repeat risk-margin measurements over each one-year period along the maturity. We used the binomial discretization for a general form of the underlying risk drivers to find the continuous-time limit of the TCMC actuarial prices. We provided an interpretation specifically for the Variance and StandardDeviation prices. We showed how time consistency and market consistency change the drift and dynamics of the underlying actuarial and financial risks, and then related them through possible correlations. In addition to these two actuarial prices, we also provided the best-estimate value calculated using the discounted expected value of the payoff to compare the risk margin and loading included in actuarial prices.

We then turned into implementing the market-consistent price of a simple unit-linked contract without guarantee that has enough flexibility to present a realistic picture of the method. We applied the two-step actuarial valuation in one-period and multi-period settings. We first presented the EIOPA price and its formulation for independent financial and actuarial risks. We then provided the time-consistent version of the two-step actuarial operator and used the result of Chapter 2 to present the continuous-time limit of the price when actuarial and financial risks are independent. For dependent risks, it is not possible to provide an analytical solution. Next, we provided a numerical scheme for implementing two-step actuarial valuations regardless of the dependence structure of financial and actuarial risks. The contract has two main risk drivers: the market value of the assets/equity as financial risk and the longevity risk of an individual or a cohort entered in the contract as actuarial risk. The market value of the assets or equity is modeled using geometric Brownian Motion (GBM), priced in a complete market with a no-arbitrage argument. Longevity risk is modeled using the famous Lee-Carter model. Both models are constructed in a diffusion setting without jump. To calculate the EIOPA and time-consistent prices, we 
need to repeat numerous one-period conditional operators. To provide them, we used the well-known Least-Square Monte-Carlo (LSMC) and Regression Later methods to calculate prices. In each case, we then explained how they should be adapted to correspond to the two-step operator. We showed practitioners that the methods outlined to calculate the market-consistent value are easy to implement for different practical valuation problems, particularly for pension and insurance liabilities. Finally, we presented the numerical results and compared the best-estimate, EIOPA risk-margin, and time-consistent values of the market-consistent price. We showed that the two-step operator guarantees market consistency and reflects the possibility of (partially) hedging actuarial risk if it depends on financial risk in the complete market. We showed that both EIOPA and time-consistent versions of the market-consistent prices correspond to a risk-adjusted best-estimate price, if the actuarial risk is perfectly correlated with the financial risk.

\subsection{Market-consistent Valuation of the Participating Pension Policy}

We provided a time-consistent and market-consistent valuation of the participating pension contract with a guaranteed annual return and a profit-sharing mechanism. The payoff of the participating contract is a combination of the financial risks such as investment asset, and interest rate risk and actuarial risks such as mortality/ longevity. Similar to other insurance products, the liabilities imposed by these products are subject to be valued in a market-consistent setting. Since there is an underlying unhedgeable actuarial risk (mortality/longevity) in the pension contracts, the actuarial value of the contract should be considered while the financial risk should be addressed through a financial pricing framework. If there is any correlation between the actuarial and financial risks to form the final payoff, the price must reflect the possible partial hedging for the actuarial part through financial market dynamics. This can be provided by the time-consistent two-step valuation that we implemented in Chapter 4.

We assumed that the hybrid payoff is a combination of the investment on equity, interest rate, and the mortality/longevity risks; for simplicity the volatility is held constant. We modeled the investment asset in a Black-Scholes framework with a Geometric Brownian motion (GBM), the Hull-White short rate model for the interest rate risk, and the LeeCarter model for mortality/longevity risk and we valuated the discounted payoff with the backward iteration of the two-step actuarial operator over the valuation period. Moreover, the payoff and the overall liability connected to the participating contract is path dependent through the crediting mechanism. We developed the crediting and profit-sharing from a pure financial mechanism in Grosen and Jorgensen (2000) to a hybrid financialactuarial mechanism. We introduced a hybrid funding ratio that is multiplied with the 
updated ratio of the number of survivors at maturity. This gives a more realistic image of the funding evolution where the status of the survivors also play a role to credit (or not credit) interest rate for the policy reserves. The time-consistent two-step operator provides a useful framework to capture the dynamic nature of the hybrid liability we have to value. To numerically calculate the price of the contract, we focused on the finite difference intervals as the main setup for discretization of the underlying process and the payoff functions. Note that the payoff is path-dependent, and the backward iteration of the operator over the long-term valuation period imposes a huge load on the calculation, especially in higher dimensions. To facilitate more speed and efficiency, we used the Least Square Monte Carlo (LSMC) and regress-later method.

The numerical implementation reflects a diverse result of the time-consistent and market-consistent actuarial prices for the participating contract, when different parameters and risk drivers vary. We compared the time-consistent price to other alternative market-consistent prices, like best-estimate and one-period two-step actuarial value, and represent the risk-loading and the effect of the time-consistency by its risk-premium. We observed that the major part of the actuarial risk-loading is due to the time-consistency risk-premium, and a very small portion of it comes from the one-period loading over the expected value. The results also show that the volatility of the asset return can increase the price. This increase is more significant for the longer maturities and for more liberal profit-sharing policies with higher distribution ratio and lower required level of the funding ratio. On the other hand, the volatility of the interest rate dynamics also affect the price. We also studied the effect of the stochastic interest rate by comparing the results provided by different levels of the interest rate volatility and deterministic term structure. The results show that for the short maturities, the time-consistent and market-consistent actuarial price is not sensitive to the randomness of the interest rate. For the longer maturities, the price is highly sensitive to the higher levels of the interest rate volatility. However for small $\sigma_{r}$ in the Hull-White model, the stochastic interest rate does not make a significant difference in the market-consistent price as compared to the deterministic version.

\subsection{Perspectives for Future Research}

There are some interesting questions on the market-consistent actuarial valuation that might worth further research. Most of the questions are raised from the current research framework, but not studied in this thesis because of the limitations in time and the scope of the project.

\section{- Incomplete market for the financial risk(s):}

The first idea is that, what if the market for the financial risks is not complete and therefore it is not possible to perfectly hedge the financial position. When the fi- 
nancial market is complete, we could hedge part of the actuarial risk (in incomplete market) via its correlation to the hedgeable financial risk driver. What happens if we don't have this possibility anymore? In fact, if the financial risk is partially hedgeable, how much of the correlated actuarial risk is hedgeable? How the market-consistent price changes under incomplete market for financial risk? In the normal case to price a financial risk in an incomplete market one might use the minimal entropy martingale measure. The similar situation is discussed in Dhaene et al. (2015) and with focus on the dependence structure of the measures with which the financial and actuarial risks are valuated.

\section{- Is the two-step operator still needed?}

Suppose we use the minimal entropy martingale measure for the financial risk traded in the incomplete market. The next question is: under the minimal entropy martingale measure, is the two-step actuarial valuation still needed to build the market-consistent price? If yes, how it should be adapted? how should we implement the separation of the actuarial and financial information flow?

\section{- More than one financial risk in incomplete market:}

Note that, we might have more than one financial risk driver. Let us say for example, if we have two financial risk drivers that the market might be incomplete for each of them or both. What will happen for the minimal entropy measure? Is it possible to find an actuarial pricing operator equivalent to the minimal entropy martingale measure? If yes, one might say if the market for one of the financial risks is still complete and accordingly hedgeable (and incomplete for the other one and therefore unhedgeable), we might threat the unhedgeable financial risk similar to the unhedgeable actuarial risk, and valuate it via the actuarial pricing operator. Therefore in the two-step valuation, we have two unhedgeable risks valuable by the actuarial operator in the inner step, and one hedgeable risk to be valuated by the expected value under the unique martingale measure $\mathbb{Q}$.

\section{- How to construct time-consistency when the financial market is incomplete?}

When the market for the financial risk(s) is incomplete, to what extent can the market-consistent pricing operator be extended also to the time-consistent price. Is it possible to apply the backward iteration of the one-period valuation? How much the resulting TCMC price will be different than the best-estimate? How much will be the relative time-consistency risk premium?

\section{- Continuous-time limit of TCMC with jump process:}


In the binomial discretization of the underlying risk drivers we used the simple diffusion processes to model the financial and actuarial risk. How the continuoustime limit of the time-consistent and market-consistent price will change if we add a jump component to the diffusion models? What will be the risk-adjusted drift and diffusion coefficient of the underlying actuarial risk drivers? How the possible hedge cost will change?

\section{- Market-consistent valuation of the participating policy with surrender option:}

To price the participation pension policy we ignored the possibility of the surrender option and lapsation. We also didn't price it under EIOPA risk-margin. One interesting research can be, adding these options to the contract and price it again with time-consistent and EIOPA risk margin and make a comparison. It is important to know, how much the surrender option will add to the market-consistent price? 


\section{Bibliography}

Acciaio, B. and Penner, I. (2011). Dynamic convex risk measures. Advanced Mathematical Methods for Finance, pages 1-34.

Amin, K. I. (1993). Jump diffusion option valuation in discrete time. The Journal of Finance, 48(5):1833-1863.

Angelis, P. D., Martire, A. L., and Russo, E. (2014). A bivariate model for evaluating equitylinked policies with surrender option. Scandinavian Actuarial Journal.

Applebaum, D. (2004). Lévy Processes and Stochastic Calculus (Cambridge Studies in Advanced Mathematics). Cambridge University Press, Cambridge.

Artzner, P., Delbaen, F., Eber, J., and Heath, D. (1999). Coherent measures of risk. Mathematical Finance, 9(3):203-228.

Artzner, P., Delbaen, F., Eber, J., Heath, D., and Ku, H. (2007). Coherent multiperiod risk adjusted values and bellman's principle. Annals of Operations Research, 152(1):5-22.

Bacinello, A. R. (2003). Pricing guaranteed life insurance participating policies with annual premiums and surrender option. North American Actuarial Journal, 7:1-17.

Bacinello, A. R., Biffis, E., and Millossovich, P. (2010). Regression-based algorithms for life insurance contracts with surrender guarantees. Quantitative Finance, 10:1077-1090.

Bacinello, A. R., Millossovich, P., Olivieri, A., and Pitacco, E. (2011). Variable annuities: A unifying valuation approach. Insurance: Mathematics and Economics, 49:285-297.

Barndorff-Nielsen, O. E., Mikosch, T., and Resnick, S. I. (2001). Levy Processes; Theory and Applications. Birkhauser, c/o Springer-Verlag, New York.

Barrieu, P. and El Karoui, N. (2009). Pricing, hedging, and designing derivatives with risk measures. In Carmona (2009).

Baule, R. and Wilkens, M. (2004). Lean trees-a general approach for improving performance of lattice models for option pricing. Review of Derivatives Research, 7(1):53-72.

Bernard, C., Courtois, O. L., and Quittard-Pinon, F. (2005). Market value of life insurance contracts under stochastic interest rates and default risk. Insurance: Mathematics and Economics, 36:499-516. 
Biffis, E. (2005). Affine processes for dynamic mortality and actuarial valuations. Insurance: Mathematics and Economics, 37(3):443-468.

Bion-Nadal, J. (2008). Dynamic risk measures: Time consistency and risk measures from BMO martingales. Finance and Stochastics, 12(2):219-244.

Bion-Nadal, J. (2009). Time consistent dynamic risk processes. Stochastic Process Appl., $119(2): 633-654$.

Black, F. and Scholes, M. (1973). The pricing of options and corporate liabilities. Journal of Political Economy, 81:637-659.

Bühlmann, H. (1970). Mathematical methods in risk theory. Springer Verlag, Berlin.

Cairns, A., Blake, D., and Dowd, K. (2006). Pricing death: Frameworks for the valuation and securitization of mortality risk. Astin Bulletin, 36(1):79.

Cairns, A. J., Blake, D., Dowd, K., Coughlan, G. D., Epstein, D., and Khalaf-Allah, M. (2011). Mortality density forecasts: An analysis of six stochastic mortality models. Insurance: Mathematics and Economics, 48:355-367.

Cairns, A. J. G., Blake, D., Dowd, K., Coughlan, G. D., Epstein, D., Ong, A., and Balevich, I. (2009). A quantitative comparison of stochastic mortality models using data from england and wales and the united states. North American Actuarial Journal, 13(1):1-35.

Carmona, R. (2009). Indifference pricing: theory and applications. Princeton University Press.

Carriere, J. (1996). Valuation of the early-exercise price for options using simulations and nonparametric regression. Insurance: mathematics and Economics, 19(1):19-30.

Chen, H. and Cox, S. H. (2009). Modeling mortality with jumps: Applications to mortality securitization. Journal of Risk and Insurance, 76(3):727-751.

Cheridito, P., Delbaen, F., and Kupper, M. (2005). Coherent and convex monetary risk measures for unbounded cadlag processes. Finance and Stochastics, 9(3):369-387.

Cheridito, P., Delbaen, F., and Kupper, M. (2006a). Coherent and convex monetary risk measures for unbounded cadlag processes. Finance and Stochastics, 9(3):369-387.

Cheridito, P., Delbaen, F., and Kupper, M. (2006b). Dynamic monetary risk measures for bounded discrete-time processes. Electronic Journal of Probability, 11(3):57-106.

Cheridito, P. and Stadje, M. (2009). Time-inconsistency of var and time-consistent alternatives. Finance Research Letters, 6(1):40-46. 
Cont, R. and Tankov, P. (2012). Financial modelling with jump processes. Chapman and Hall/ CRC Press, London, 2nd, illustrated, revised edition.

Cummins, D. J. (1990). Asset pricing models and insurance ratemaking. ASTIN Bulletin, 20(2):125-166.

Cvitanic, J. and Karatzas, I. (1992). Convex duality in constrained portfolio optimization. Annals of applied probability, 2(4):767-818.

Darcy, S. P. (1989). On becoming an actuary of the third kind. Proceedings of the Casualty Actuarial Society, 145:45-76.

Delbaen, F. and Schachermayer, W. (1994). A general version of the fundamental theorem of asset pricing. Mathematische Annalen, 300(3):463-520.

Delbaen, F. and Schachermayer, W. (1996). The variance-optimal martingale measure for continuous processes. Bernoulli, pages 81-105.

Delong, L. (2013). Backward Stochastic Differential Equations with Jumps and Their Actuarial and Financial Applications. Springer-Verlag, London.

Dhaene, J., Stassen, B., Devolder, P., and Vellekoop, M. (2015). The minimal entropy martingale measure in a market of traded financial and actuarial risks. Journal of Computational and Applied Mathematics, 282:111-133.

Duan, J.-C., Gauthier, G., and Simonato, J.-G. (2003). A markov chain method for pricing contingent claims. Stochastic Moddeling and Optimization, with application in Queues, Finance, and Supply Chains, pages 333-362.

EC Delegated Regulation 2015/35 (2015). Commission delegated regulation (eu) 2015/35. Technical report, European Parliament and of the Council on the taking-up and pursuit of the business of Insurance and Reinsurance (Solvency II).

EIOPA (2010). QIS5 technical specifications. Technical report, European Insurance and Occupational Pensions Authority, [https://eiopa.europa.eu].

Figlewski, S. and Gao, B. (1999). The adaptive mesh model: a new approach to efficient option pricing. Journal of Financial Economics, 53:313-351.

Figueroa-López, J. E. F.-L. (2012). Jump-diffusion models driven by lévy processes. Handbook of Computational Finance, Springer Handbooks of Computational Statistics:6188.

Filipovic, D. and Vogelpoth, N. (2008). A note on the Swiss solvency test risk measure. Insurance: Mathematics and Economics, 42(3):897-902. 
Föllmer, H. and Penner, I. (2006). Convex risk measures and the dynamics of their penalty functions. Statistics and decisions, 24(1):61-96.

Föllmer, H. and Schweizer, M. (1989). Hedging by sequential regression: An introduction to the mathematics of option trading. ASTIN Bulletin, 18(2):147-160.

Frittelli, M. and Gianin, E. R. (2004). Dynamic Convex Risk Measures, Risk measures for the 21st Century. Wiley, New York.

Gerber, H. (1979). An introduction to mathematical risk theory. Irwin Homewood.

Glasserman, P. (2004). Monte Carlo Methods in Financial Engineering. Springer-Verlag, New York.

Glasserman, P. and Yu, B. (2004a). Number of paths versus number of basis functions in american option pricing. The Annals of Applied Probability, 14(4):2090-2119.

Glasserman, P. and Yu, B. (2004b). Pricing american options by simulation: Regression now or regression later? In Niederreiter, H., editor, Monte Carlo and Quasi-Monte Carlo Methods in Scientific Computing, page 213Ü226. Springer Verlag, Berlin.

Goovaerts, M. and Laeven, R. (2008). Actuarial risk measures for financial derivative pricing. Insurance: Mathematics and Economics, 42(2):540-547.

Grosen, A. and Jorgensen, P. L. (2000). Fair valuation of life insurance liabilities: The impact of interest rate guarantees, surrender options, and bonus policies. Insurance: Mathematics and Economics, 26:37-57.

Hamada, M. and Sherris, M. (2003). Contingent claim pricing using probability distortion operators: methods from insurance risk pricing and their relationship to financial theory. Applied Mathematical Finance, 10(1):19-47.

Hanewald, K., Post, T., and Gründl, H. (2011). Stochastic mortality, macroeconomic risks and life insurer solvency. The Geneva Papers on Risk and Insurance - Issues and Practice, 36:458-475.

Henderson, V. (2002). Valuation of claims on nontraded assets using utility maximization. Mathematical Finance, 12(4):351-373.

Henderson, V. and Hobson, D. (2009). Utility indifference pricing: An overview. In Carmona (2009).

Hilliard, J. E. and Schwartz, A. (2005). Pricing european and american derivatives under a jump-diffusion process: A bivariate tree approach. Journal of Financial and Quantitative Analysis, 40(3):671-691. 
Hobson, D. (2004). Stochastic volatility models, correlation, and the $q$-optimal measure. Mathematical Finance, 14(4):537-556.

Hodges, S. and Neuberger, A. (1989). Optimal replication of contingent claims under transaction costs. Review of Futures Markets, 8(2):222-239.

Hull, J. and White, A. (1996). Using hull-white interest rate trees. The Journal of Derivatives, 3(3):26-36.

Jang, J. (2007). Jump diffusion processes and their applications in insurance and finance. Insurance: Mathematics and Economics, 41(1):62-70.

Jobert, A. and Rogers, L. (2008). Valuations and dynamic convex risk measures. Mathematical Finance, 18(1):1-22.

Kaas, R., Goovaerts, M., Dhaene, J., and Denuit, M. (2008). Modern Actuarial Risk Theory: Using R. Springer Verlag.

Keller, P. and Luder, T. (2004). White paper of the swiss solvency test. Technical report, Swiss Federal Office of Private Insurance (BPV), Bern, Switzerland.[http://www.bpv.admin.ch/de/pdf/white_paper_sst.pdf].

Kleinow, T. (2009). Valuation and hedging of participating life-insurance policies under management discretion. Insurance: Mathematics and Economics, 44:78-87.

Kloeden, P. E. and Platen, E. (1999). Numerical Solution of Stochastic Differential Equations., volume 23 of Applications of Mathematics. Berlin, Heidelberg: Springer-Verlag.

Korn, R., Korn, E., and Kroisandt, G. (2010). Monte Carlo Methods and Models in Finance and Insurance. CRC Press, London.

Kramkov, D. and Schachermayer, W. (1999). The asymptotic elasticity of utility functions and optimal investment in incomplete markets. Annals of Applied Probability, 9(3):904950.

Kupper, M., Cheridito, P., and Filipovic, D. (2008). Dynamic risk measures, valuations and optimal dividends for insurance. In Mini-Workshop: Mathematics of Solvency. Mathematisches Forschungsinstitut Oberwolfach.

Kushner, H. J. and Dupuis, P. (2001). Numerical Methods for Stochastic Control Problems in Continuous Time (Stochastic Modelling and Applied Probability 24), 2nd Ed. SpringerVerlag, New York.

Laeven, R. J. A. and Stadje, M. (2014). Robust portfolio choice and indifference valuation. Mathematics of Operations Research, forthcoming. 
Lee, R. D. and Carter, L. R. (1992). Modeling and forecasting u.s. mortality. Journal of the American Statistical Association, 87(419):659-671.

Li, J. and Szimayer, A. (2014). The effect of policyholders? rationality on unit-linked life insurance contracts with surrender guarantees. Quantitative Finance, 14:327-342.

Longstaff, F. A. and Schwartz, E. S. (2001). Valuing american options by simulation: A simple least-squares approach. The Review of Financial Studies, 14(1):113-147.

Létourneau, P. and Stentoft, L. (2014). Refining the least squares monte carlo method by imposing structure. Quantitative Finance, 14:495-507.

Maccheroni, F., Marinacci, M., and Rustichini, A. (2006). Ambiguity aversion, robustness, and the variational representation of preferences. Econometrica, 74(6):1447-1498.

Madan, D. and Milne, F. (1994). Contingent claims valued and hedged by pricing and investing in a basis. Mathematical Finance, 4(3):223-245.

Malamud, S., Trubowitz, E., and Wüthrich, M. (2008). Market consistent pricing of insurance products. ASTIN Bulletin, 38(2):483-526.

Merton, R. C. (1976). Option pricing when underlying stock returns are discontinuous. Journal of Financial Economics, 3(1):125-144.

Møller, T. (2002). On valuation and risk management at the interface of insurance and finance. British Actuarial Journal, 8(4):787-827.

Monoyios, M. (2004). Option pricing with transaction costs using a markov chain approximation. Journal of Economic Dynamics \& Control, 28(5):889-913.

Monoyios, M. (2006). Characterisation of optimal dual measures via distortion. Decisions in Economics and Finance, 29(2):95-119.

Musiela, M. and Zariphopoulou, T. (2004). An example of indifference prices under exponential preferences. Finance and Stochastics, 8(2):229-239.

Musiela, M. and Zariphopoulou, T. (2004b). A valuation algorithm for indifference prices in incomplete markets. Finance Stochastic, 8(3):399-414.

Musiela, M. and Zariphopoulou, T. (2009). The single period binomial model. In Carmona (2009).

Øksendal, B. (2003). Stochastic differential equations: an introduction with applications. Springer Verlag.

Pelsser, A. (2000). Efficient methods for valuing interest rate derivatives. Springer Verlag. 
Pelsser, A. (June 2011). Pricing in incomplete market. Netspar panel papers, (25).

Pelsser, A. and Salahnejhad, A. (2016). Time-consistent actuarial valuation. Insurance Mathematics and Economics, 66:97-112.

Pelsser, A. and Stadje, M. (2014). Time-consistent and market-consistent evaluations. Mathematical Finance, 24(1):25-65.

Peng, S. (2004). Filtration consistent nonlinear expectations and evaluations of contingent claims. Acta Mathematicae Applicatae Sinica (English Series), 20(2):191-214.

Platen, E. (2006). A benchmark approach to finance. Mathematical Finance, 16(1):131151.

Platen, E. and Heath, D. (2006). A Benchmark Approach to Quantitative Finance: A Benchmark Approach. Springer Verlag.

Pratt, J. (1964). Risk aversion in the small and in the large. Econometrica, 32(1):122-136.

Renshaw, A. E. and Haberman, S. (2006). A cohort-based extension to the lee-carter model for mortality reduction factors. Insurance: Mathematics and Economics, 38:556-570.

Riedel, F. (2004). Dynamic coherent risk measures. Stochastic Processes and their Applications, 112(2):185-200.

Rogers, L. (2001). Duality in constrained optimal investment and consumption problems: a synthesis. In Workshop on Financial Mathematics and Econometrics held in Montreal. Springer.

Rosazza Gianin, E. (2006). Risk measures via g-expectations. Insurance Mathematics and Economics, 39(1):19-34.

Salahnejhad, A. and Pelsser, A. (2016). Market-consistent actuarial valuation with an application to eiopa risk-margin and time-consistent pricing. Working paper, Graduate School of Busness and Economics, Maastricht University.

Schmeidler, D. (1989). Subjective probability and expected utility without additivity. Econometrica, 57:571-587.

Schweizer, M. (1995). On the minimal martingale measure and the föllmer-schweizer decomposition. Stochastic analysis and applications, 13(5):573-600.

Shreve, S. E. (2010). Stochastic Calculus for Finance II, Continuous-Time Models. Springer Finance, New York. 
Stadje, M. (2010). Extending dynamic convex risk measures from discrete time to continuous time: A convergence approach. Insurance: Mathematics and Economics, 47(3):391404.

Tang, Y. and Li, B. (2007). Quantitative Analysis, Derivatives Modelling, And Trading Strategies (In the Presence of Counterparty Credit Risk for Fixed-Income Market). World Scientific Publishing Co. Re. Ltd., Singapore.

Tanskanen, A. J. and Lukkarinen, J. (2003). Fair valuation of path-dependent participating life insurance contracts. Insurance: Mathematics and Economics, 33:595-609.

Technical Specifications for the Preparatory Phase (Part 1) (2014). Technical specifications for the preparatory phase (part 1). Technical report, European Insurance and Occupational Pensions Authority.

Venter, G. G. (1991). Premium implications of reinsurance without arbitrage. ASTIN Bulletin, 21(2):223-230.

Verrall, R. J. and Wüthrich, M. V. (2012). Reversible jump markov chain monte carlo method for parameter reduction in claims reserving. North American Actuarial Journal, 16(2):240-259.

Wang, S. S. (2002). A universal framework for pricingg financial and insurance risks. ASTIN Bulletin, 32(2).

Yaari, M. E. (1987). The dual theory of choice under risk. Econometrica, 55(1):95-115.

Yin, G. G. and Zhang, Q. (2012). Continuous-Time Markov Chains and Applications: A Two-Time-Scale Approach (Stochastic Modelling and Applied Probability 37), 2nd Ed. Springer, New York.

Young, V. R. and Zariphopoulou, T. (2002). Pricing dynamic insurance risks using the principle of equivalent utility. Scandinavian Actuarial Journal, 2002(4):246-279.

Yuen, F. L. and Yang, H. (2009). Option pricing in a jump-diffusion model with regime switching. ASTIN Bulletin, 39(2):515-539.

Zaglauer, K. and Bauer, D. (2008). Risk-neutral valuation of participating life insurance contracts in a stochastic interest rate environment. Insurance: Mathematics and Economics, 43:29-40. 


\section{Summary}

The main body of this thesis discusses and introduces the methodologies to implement the "market-consistent" actuarial valuation for a hybrid liabilities consisting the underlying financial and actuarial risks. In that respect, we give a clear picture on what happens in the valuation procedure to make the pricing operator market-consistent, and how the information flow should be dealt with over the valuation period. We have provided both analytical and numerical implementations for the valuation methodology, and we have compared the results with some available methods used in industry.

The market-consistent actuarial price requires the time-consistency property to avoid the arbitrage opportunities over the valuation period. This is similar to build the tower property for a non-linear pricing operator. Time-consistent pricing operators can be created by backward iteration of one-period valuations. In Chapter 2 of this thesis, we investigate the continuous-time limits of the well-known actuarial premium principles when such backward iteration procedures are applied. This method is applied to an insurance risk process in the form of a diffusion process. We show that in the case of the diffusion process, the one-period time-consistent Variance premium principle converges to the non-linear exponential indifference price. Furthermore, we show that the StandardDeviation and the Cost-of-Capital principle converge to the same price limit. In chapter 3 , we consider the heavy-tailed nature of the insurance liabilities as a motivation to model the actuarial risk under the jump-diffusion process and get a more realistic picture of the underlying risk dynamics. We generalize the backward iteration of the one-period valuation of the insurance premium principles when the unhedgeable insurance risk can also jump by an stochastic arrival time. As a result, we no longer observe that the different premium principles converge to the same limit since each principle reflects the effect of the jump differently. In the Cost-of-Capital principle in particular, the VaR operator fails to capture the jump risk for small jump probabilities and the time-consistent price depends only on the distribution of the premium jump.

Market-Consistent valuation is a requirement by the EU insurance supervisor to valuate the insurance liabilities such as life and pension contracts that are not (actively) traded in the market. To obtain a market-consistent price, in Chapter 4, we combine the hedgeable financial risk with an (partially) unhedgeable actuarial risk and price the hybrid payoff using a "two-step market evaluation". In a general setting, the valuation process comprises the no-arbitrage price of pure financial risk, the value of partially hedged actuarial risk attributable to its correlation with financial risk (if available), and finally the value of pure actuarial risk through well-known actuarial premium principles. We 
implement a two-step valuation using a backward iteration method and obtain a timeconsistent market-consistent (TCMC) price during the valuation period. We also provide a continuous-time limit of the TCMC price for the Variance and Standard-Deviation actuarial prices. We also provide a market-consistent version of alternative pricing methods: the Best-Estimate pricing method typically used for pension liabilities and the EIOPA's RiskMargin method used under Solvency II to value life insurance liabilities. By comparing these prices with the TCMC price for a unit-linked contract, we show that the EIOPA RiskMargin method acts in the correct direction to reflect part of the uncertainty attributable to the future dynamics of non-hedgeable risks, whereas Best-Estimate pricing completely ignores that uncertainty. Because the Risk-Margin method still ignores certain uncertainties, it is not fully time consistent and its gap with TCMC should not be ignored for longdated contracts. In Chapter 5, we implement the two-step actuarial operator for a simple unit-linked contract with the equity and mortality, respectively as the underlying financial and actuarial risk drivers. We provide some insights into formulation of the EIOPA riskmargin and time-consistent price with the two-step actuarial operator under independence assumption. To perform the numerical calculations we adapt a customized version of the Least Square Monte Carlo (LSMC) method and simulate the three market-consistent prices (the two above and the best-estimate price). We show that the two-step actuarial valuation captures partial (or perfect) hedging as we observe that all three prices converge to one adjusted Best-Estimate price when the correlation between financial and actuarial risks increases.

In Chapter 6 we consider the market-consistent valuation of the participating pension contract. It is an example of the long-dated liabilities that are not fully traded and hedgeable in the market. Due to the existence of actuarial risk in their payoff, they are normally priced by non-linear premium principles. Such long-term positions make the issuer's valuation and risk management vulnerable to the dynamics of medium-term fluctuations. To reflect the effect of the medium-time evolution on the price, we require time-consistency while the premium principles are not time-consistent. The study provides a TSMC pricing method for a participating life/pension scheme with guaranteed interest rate. We consider a hybrid combination of the mortality, interest rate and equity risks in the final payoff and the profit-sharing mechanism over the life of the contract. We use the Least-Square Monte-Carlo (LSMC) method to implement the backward iteration and calculate the conditional expectations in the two-step operator. We report the TCMC price and compare it to the expected value of the discounted payoff and measure the relative risk loading and time-consistency risk premium. We also study the effect of the stochastic interest rate on the price of the contract as compared to the deterministic one. 


\section{Acknowledgements}

I would like first to thank my supervisor Prof. dr. Antoon Pelsser for his creative and smart scientific mind to find solutions in the time of problems, his flexibility and his commitment to put me in a situation that made me learn how to research and grow independently in the academic environment. I also thanks my co-supervisor Dr Thomas Post whose valued comments and friendly guidance made considerable improvements on my papers.

My sincere appreciation goes to Prof. dr. Jan Dhaene who has been not only supporting my academic and professional work, but also has been a fantastic friend during the last years. I have learned a lot from his scientific ideas on actuarial sciences, but much more from his attitude to life, humanity and openness. We discussed about many things from insurance to politics, from travel to religion and from happiness to sorrow. We still have a lot of plans and discussion points in our minds for the rest of our friendship in which disagreement has been never ended up in censorship. But the main lesson that we remind and validate always, is that "we should always leave the doors open!".

My special thanks to my wife Somaieh that accompanied me in this long journey of doing a PhD. Your patience and sympathy when I was complaining about the problems I came across, took all the negative energies away and opened my way to progress further. Thank you for experiencing the difficulties of this scientific work with me.

I would also like to express my gratitude to other members of the assessment committee who devoted their time to read my papers and provided valuable feedback for further improvements. Prof. dr. Dennis Bams, the chair of the committee, Prof. dr. Michel Vellekoop and Prof. dr. Peter Schotman, it is my honor to get approval on my scientific work from you and have your scientific credits behind my thesis.

Maastricht, my nice and cozy city, I am very grateful to you to give me the chance to meet wonderful people, to share unforgettable memories, seeing familiar faces, and to see breathtaking scenery. Leaving you has been one of the saddest moments when you were giving me the feeling of home.

I would like to extend my heartfelt gratitude and deep devotion to those who always gave me not only a deep motivation to continue my education and academic carrier, but also important values that painted my growth as a child and adult with nice colors; Daie Aziz, Daie Rahim, Amou Hossein and Parvaneh, thank you so much.

Good friends are hard to find, harder to leave and impossible to forget; Bijan and Hoda, in the last four years, you have been always among my bests. My special thanks to Bijan who assisted me on my thesis whenever I needed help and without his contribution in the last days, I would not be able to handle preparation of my thesis and defense. Livy and Cigdem, your lifestyle and attitude to life have been very inspiring to me, thank you for your patience against my venting about difficulties and hardships and thanks for your 
hospitality and making one of my best memories ever, in Greece. Jarret, man! During the difficult days of my $\mathrm{PhD}$, despite your own problems and difficulties, you always gave effective and strong support from distance. That was an art; thank you so much heartily for your true friendship. Nasser, if you are not busy with your social life and special breakfast ceremonies, we shared many good memories, happy and sad moments together. Shuan, you have been always the one whom I could rely on his friendship, information and helps. Pouyan, thanks for your positive energy and optimism and I apologize to steal Nasser's energy to upgrade mine ;). Iman the Great, Iman the little, and Ehsan, I will never forget our not very tasty lunches in Mensa, but the nice landscapes we were always seeing around that brought a lot of joy to our gatherings. Khaleh Mitra, Agha Sohrab, Agha Davar and Nasrin Khanoom and all my other Iranian friends, whenever I see you I feel like I am in my family with the same warmth, intimacy, and joy.

My special gratitude goes to all my non-Iranian friends and colleagues, whom I learned a lot from them. Special thanks to my colleague Kouma, for all we shared as memories, experiences and problems; I hope you a lot of success. Anne van Delft, Anna Zseleva, Sofya jan, Valentina, Elena, Karin, Yolanda and all others, thank you so much for all your kindness.

Jo Haesen, I never consider you as my landlord, but my Dutch friend. The friendship started when the first time we met and only shook the hands instead of writing a contract. Thank you very much for always being available for us, for all concerts, all the rides, all diners, all the patience you had, all chats and all your hospitality and understanding.

It is all about being good neighbors; thank you Jo and Jessi Robroek for being great neighbors. The care that you have been sharing with me and others are amazing; we will drink together again. Guido, Natasha, and Dirk, I hope I could come back each summer to Vroenhoven to taste your wonderful cherries. Carine and Piere, thanks for the great chats that we had during last years.

Last but not least, my parents who paved my way to be what I am now. Thank you Mom for all the nights that you stayed up with me, for all the cares and worries that you tried to hide them, and for all your love. I know how much you want to see your son in this end, and you know that your presence in my defense ceremony was one of the biggest motivations for me to start and finish this PhD. Thank you Dad, for all the years that were working very hard to make my life better. I am sure you are now happy with where I am standing and I am also happy to see your smile. My brothers Arshad, Parviz and Cyrus and my lovely sister Maryam, I know as the little brother, I was in the center of the attention, that you deserved more, I am so thankful of your permanent support. 


\section{About the Author}

Ahmad Salahnejhad Ghalehjooghi was born on July 20, 1980 in Tehran, Iran. He holds a BSc. in Statistics (2003, Shahid Beheshti University, Tehran), a MSc. in Actuarial Science (2007, Allameh Tabatabai University, Tehran) and a MSc. in Finance - Risk Management (2012, VU University and Duisenberg School of Finance, Amsterdam). He has also completed a Leadership program in Finance at Duisenberg School of Finance. He started his professional life in 2005 in Parsian Insurance Company of Iran as a life actuary to carry out premium calculation and reserving studies and was involved to develop the business for the company along with the

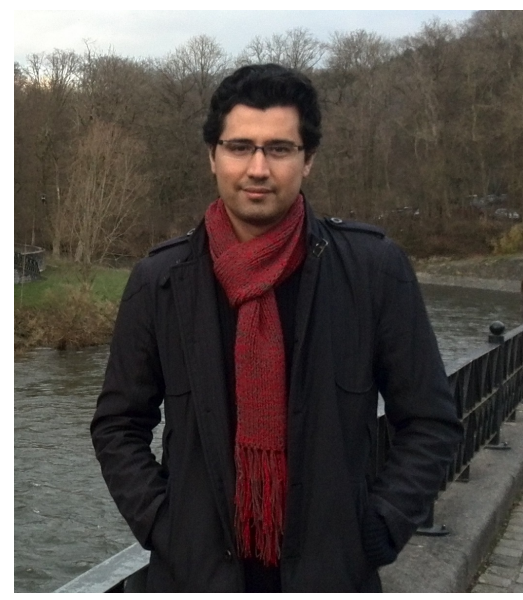
required IT infrastructure. In 2009, he was promoted as the life insurance manager and helped the company to develop the life business, before he left to continue his education in the Netherlands, in 2011. He was involved in different activities such as membership of the life insurance development committee of Iranian Central Insurance and establishment of two new insurance companies. He taught different courses in life insurance and basic concepts in actuarial sciences for different organizations in Iran.

He started the PhD in Actuarial Science at Graduate School of Business and Economics in Maastricht University in September 2012 and worked under supervision of Prof. Dr. Antoon Pelsser. He worked on the actuarial pricing/valuations and became specialized on techniques to implement the Time-Consistent and Market-Consistent valuations for different insurance liabilities. He did tutoring in Finance together with supervision of bachelor and master theses. Meanwhile, he was also a researcher of HPCFinance project in Marie-Curie ITN and his research was funded by European Union Seventh Framework Programme [FP7/2007-2013]. His PhD ended up with three papers on time-consistent and market-consistent actuarial valuations and its applications on pricing the life/pension liabilities. Ahmad also has publications on application of the extreme value theory on subadditivity of VaR and some papers in statistical methods for food contamination studies before he changed his major to actuarial science. Right now he works as an actuary in Pricing and Product Hedging department in Nationale-Nedelanden and works on the solutions for the Mortality Differentiation and the Longevity Hedging. 